\title{
Perylene Monoimide as a Versatile Fluoroprobe: The Past, Present, and Future
}

\author{
Rupam Roy ${ }^{\mathrm{a}, \mathrm{s}}$ (1) \\ Aasif Khan ${ }^{\mathrm{a}, \boldsymbol{S}}$ (D) \\ Oendrila Chatterjee ${ }^{\mathrm{a}, \mathrm{s}}$ (D) \\ Sukomal Bhunia ${ }^{\text {(iD }}$ \\ Apurba Lal Koner*a(D) \\ a Bionanotechnology Lab, Department of Chemistry, Indian Institute \\ of Science Education and Research Bhopal, Bhopal Bypass Road, \\ Bhauri, Bhopal, Madhya Pradesh, 462066, India \\ akoner@iiserb.ac.in \\ $\$$ These authors contributed equally to this work.
}

Dedicated to Prof. Heinz Langhals and Prof. Jonathan S. Lindsey.

Received: 01.06.2021

Accepted after revision: 13.07 .2021

DOI: 10.1055/a-1551-6930; Art ID: OM-2021-06-0035-REV

License terms: C) $\$$

c) 2021. The Author(s). This is an open access article published by Thieme under the terms of the Creative Commons Attribution-NonDerivative-NonCommercial License, permitting copying and reproduction so long as the original work is given appropriate credit. Contents may not be used for commercial purposes, or adapted, remixed, transformed or built upon. (https://creativecommons.org/licenses/by-nc-nd/4.0/)

Abstract Perylene dyes have transcended their role as simple colorants and have been reinvigorated as functional dyes. Based on the substitution at the peri-position by six-membered carboxylic imides, the perylene family is principally embellished with perylene diimides (PDIs) and perylene monoimides (PMIs). Perylene dyes are widely acclaimed and adorned on account of their phenomenal thermal, chemical, and photostability juxtaposed with their high absorption coefficient and nearunity fluorescence quantum yield. Although symmetric PDIs have always been in the limelight, their asymmetrical counterpart PMI is already rubbing shoulders, thanks to the consistent efforts of several scientific minds. Recently, there has been an upsurge in engendering PMIbased versatile organic architectures decked with intriguing photophysical properties and pertinent applications. In this review, the synthesis and photophysical features of various PMI-based derivatives along with their relevant applications in the arena of organic photovoltaics, photocatalysis, self-assembly, fluorescence sensing, and bio-imaging are accrued and expounded, hoping to enlighten the less delved but engrossing realm of PMIs.

Table of content:

1 Introduction

2 Advantages of PMI over PDI

3 Challenges in Working with PMI and Ways to Overcome

4 Various Aspects of Reactivity of Different Positions

5 Synthesis of the PMI core

6 Synthesis of PMI Derivatives

6.1 Bromination of PMI

6.2 Synthesis of PMI Derivatives using Coupling Reactions

6.2.1 Suzuki Coupling

6.2.2 Sonogashira Coupling

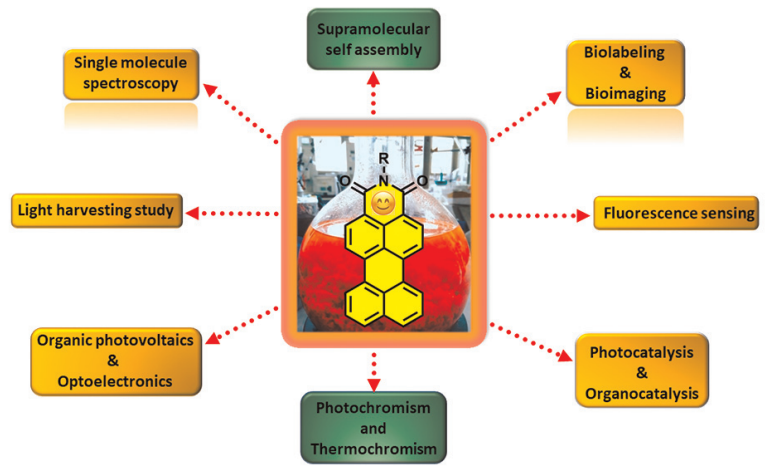

6.2.3 Buchwald-Hartwig Coupling Reaction

6.3 Nucleophilic Substitution Reactions

6.4 Peri-Annulation Reaction

7 Photophysical Properties of PMI

8 Singlet Fission Properties

9 Förster Resonance Energy Transfer with PMI

10 Symmetry Breaking Charge Transfer Properties

11 Panchromatic Light Absorption Properties of PMI

12 Acid/Base Sensitivity of PMI

13 NIR-Absorbing PMI

14 Achieving of Triplet State Using PMI

15 Solid-State Emissive PMI

16 Thermo-Responsive Materials with PMI

17 Photo-Responsive PMI Derivatives

18 Electrochemical Properties of Rylene Derivative

19 Self-Assembling Properties of PMI Derivatives

20 Applications of PMI in Solar Cells

21 PMIs in Bulk Heterojunction Solar Cells

22 PMIs in Dye-Sensitized Solar Cells

23 PMI as a Fluorescent Reporter

23.1 Application of PMI Derivatives for Bio-Imaging

23.2 Hydrophilic PMIs for Bio-Imaging

23.3 Aggregated PMI as NIR-Emissive Fluoroprobe for Bio-Imaging

24 Photocatalytic Hydrogen Generation using PMI

25 PMI-Based Organocatalysis

26 PMI Derivatives for Single-molecule Spectroscopy

27 Host-Guest Chemistry with PMI

28 Conclusions and Outlook

Key words: rylene dyes, perylene monoimide, self-assembly, fluorescent probe, functional organic materials

\section{Introduction}

The organic functional dyes with versatile properties and real applications are always demandable for global wellbeing and therefore, a conscientious study of such dyes has become an arena of significant research interest now-a- 


\section{Biosketches}
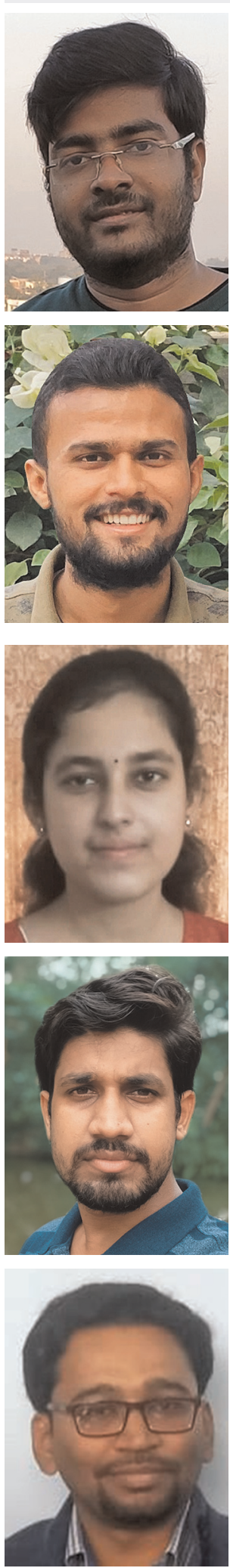

Mr. Rupam Roy received his M.Sc. degree from the Indian Institute of Technology, Guwahati in 2016. Currently, he is pursuing his doctoral degree at IISER Bhopal. His doctoral research interest is related to the design and synthesis of perylene-based materials for optoelectronic applications.

Mr. Aasif Khan received his M.Sc. degree from Jamia Millia Islamia (Central University), New Delhi in 2016. Currently, he is pursuing his doctoral research at IISER Bhopal. His doctoral research interest is related to the synthesis of new fluorescent molecules based on rylene dyes and to explore their application in various fields.

Ms. Oendrila Chatterjee received her M.Sc. degree from the University of Kalyani, West Bengal in 2018. Currently, she is pursuing her doctoral degree at IISER Bhopal. Her doctoral research interest pertains to the supramolecular photophysics of perylene-based dyes.

Mr. Sukomal Bhunia received his M.Sc. degree from Vidyasagar University, West Bengal in 2017. Currently, he is pursuing his doctoral degree at IISER Bhopal. His doctoral research interest is related to the design and synthesis of perylene monoimide-based materials for diverse applications.

Dr. Apurba Lal Koner is currently an Associate Professor at IISER Bhopal. He obtained his Ph.D. in Chemistry from Jacobs University Bremen, Germany. After postdoctoral work at the University of Oxford, he joined IISER Bhopal as a faculty member in the Department of Chemistry in 2012. One of his current research interests lies in the design and synthesis of novel fluorescent probes for sensing and fluorescence imaging. 


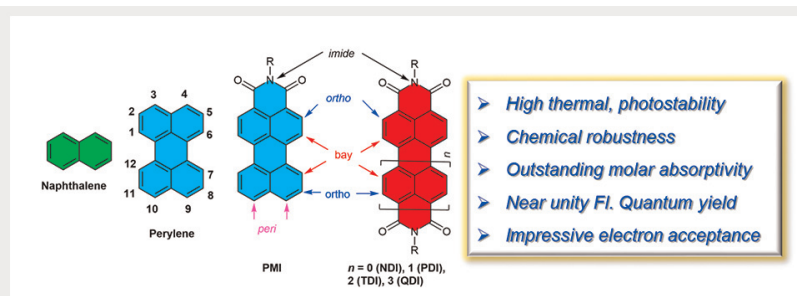

Figure 1 General structural representation of rylene dyes and their general properties.

days. ${ }^{1}$ In the large class of organic dyes, rylene imide, a class of polyaromatic hydrocarbons, has gained its recognition for nearly a century from the viewpoint of their multifaceted properties desirable for various applications. ${ }^{2}$ The rylene family represented in Figure 1, a class of polyaromatic hydrocarbons, constitutes naphthalene, perylene, terylene, and quaterrylene chromophores, wherein naphthalene is considered as the smallest member of the rylene family while the remaining ones are its higher homologues with increasing number of naphthalene units in their core. ${ }^{3}$ Generally, perylene, terylene, and quaterrylene can be prepared by connecting multiple naphthalene units via the peri-position, hence they are often referred to as oligo(peri-naphthalene). ${ }^{4}$ The intense-colored rylene pigments have been extensively used as a colorant in the car industry, also in the coloration of automotive paint and synthetic fiber, therefore one can designate them as 'superb colorant'. ${ }^{5}$ Noteworthy, naphthalene and perylene dyes have garnered immense attention from the materials chemists and have been tremendously utilized for the past few decades. ${ }^{2 b, 6}$ Based on the number of six-membered carboxylic imide moieties, perylene dye can be classified into two major categories i.e., perylene diimide (PDI) with two electron-withdrawing imide moieties symmetrically decorated on the top and bottom of the electron-rich perylene core and asymmetric perylene monoimide (PMI) possessing only one imide group. The aforementioned nomenclature is also applicable to other rylene imide dyes. Foregoing literature reports unraveled that perylene/naphthalene imides are moderate to highly soluble in common organic solvents, ${ }^{7}$ contrarily, greater hydrophobic character of terylene imide/quaterrylene imide dyes bring about a tremendous solubility problem in organic solvents because of their profound intermolecular $\pi-\pi$ stacking interaction. ${ }^{3,7 d, 8}$ In accordance with their intrinsic solubility issue, the synthesis and processing of higher rylene imide dyes (terylene, quaterrylene) are quite challenging thus restricting their application in the vast area of organic materials. Since the first discovery of PDI in 1913 by Kardos, ${ }^{9}$ this chromophore has exhibited a prodigious progression to reach an esteemed level in functional organic materials by the pioneering contributions of Langhals, Lindsey, Müllen, Würthner, Wasielewski, and other renowned scientists. ${ }^{10}$
After the invention of PDI, commercially available PDI was extensively used as an industrial pigment, therefore research on PDI was primarily focused on the tuning of their solubility, mostly by disrupting their solid-state packing rather than exploring their underlying properties that led to their crystallochromism. In the last few decades, the scientific community has carried out an extensive research to uncover other appealing properties of perylene imide derivatives such as extreme thermal and photochemical stability, chemical robustness, high molar-absorptivity in the whole UV-Vis. region, remarkable photoluminescence properties [fluorescence quantum yield (QY) near unity], and impressive electron-accepting properties. ${ }^{11}$ The 'rock-stability' of perylene imide presumably originates due to the presence of low-lying imide substituents that resist oxidation. The greater absorption coefficient of PDI compared to other classes of commonly used dyes is a reflection of its exorbitant $\pi$-conjugation throughout the whole polyaromatic scaffold. The parent PDI and its sister analogue PMI generally absorb in the visible $(400-550 \mathrm{~nm})$ region coupled with high molar-extinction coefficients in the range of $10^{4} \cdot \mathrm{M}^{-1} \cdot \mathrm{cm}^{-1}$. Interestingly, higher rylene diimides exhibit bathochromically shifted absorption maxima with escalating molar-extinction coefficient due to greater extended conjugation in a larger $\pi$-skeleton. Müllen and co-workers have ascertained the absorption maxima of PDI, terrylene diimide (TDI), quaterrylene diimide (QDI), 5DI, and hexarylene diimide (HDI) to be $580,677,762,877$, and $953 \mathrm{~nm}$ respectively, corresponding to a $100 \mathrm{~nm}$ red-shift with each additional naphthalene unit. ${ }^{4 a}$ In harmony with the emergence of PDI's intriguing properties, the synthetic development in perylene imide field has also been phenomenal in the last few decades, which has driven them to come in the spotlight of modern dyestuff research. ${ }^{12}$ Achieving such unprecedented photophysical and electrochemical properties is deeply correlated with the new synthetic pathways of making novel PDI/PMI derivatives, the motivation for working with such a class of dye stems from this strong correlation. To a physical organic chemist, the most attractive feature of PDI/PMI will be its unique fluorescence property. Although unsubstituted PDI/PMI in solution exhibits a structured emission spectrum with marginal Stokes shift, but fortunately, their Stokes shift can be enormously extended by judicious substitution of various donor groups on bay-/peri-positions. ${ }^{13}$ Self-assembly phenomenon in presence of a bad solvent or at higher concentration is an inherent feature of PMI/PDI leading to the notorious aggregation caused quenching phenomenon. Sometimes this monomer concocts one-dimensional nanostructures with distinct morphology and photophysical properties in solution. In this context, it must be mentioned that a myriad of literature has highlighted that rylene diimide candidates are very prone to engender Jand H-type aggregates in solution, where slip-stacked J-aggregates among neighbouring PMI/PDI molecules exhibit 
bathochromic shift of absorption maxima with the enhancement of fluorescence $\mathrm{QY}$, contrarily absorption spectra become blue-shifted with diminishing of fluorescence QY in side-on-side $\mathrm{H}$-aggregation. ${ }^{14}$ Theoretical research confirms that the contrasting behavior in spectroscopic properties of rylene imide assembly arises due to distinctive mode of exciton coupling in the two different assemblies in solution. ${ }^{15}$ PMI or PDI and their higher rylene dyes serve as potential near-IR (NIR)-emissive materials that are well suited for bio-imaging. ${ }^{16}$ Several stupendous studies have disclosed that the NIR-emission from rylene imide scaffold can be accomplished in two ways - firstly, by introducing strong donor moieties along with a spacer unit in the suitable position of the PMI/PDI core, secondly, with fine control of their morphology in solution that puts forth an enthralling approach devoid of tedious synthesis. Last but not the least, controlled self-assembly via $\pi-\pi$ stacking and H-bonding among perylene imide derivatives has aided in excavating novel excited-state photophysical phenomenon, for example, excited-state symmetry-breaking charge transfer and singlet fission (SF) to name a few. ${ }^{17}$ SF from PMI/PDI embedded compounds has brought a breakthrough in achieving higher power conversion efficiency (PCE) values in perylene imide-based organic solar cells (OSCs) by overcoming the Shockley-Queisser limit. Scientists have found perylene imide dyes as a powerful alternative to fullerene in organic photovoltaics as PDI's/PMI's absorption covers the entire UV-Vis region and the reduction potential of unsubstituted $\mathrm{PDI}$ is close to $-1.0 \mathrm{~V}$ vs. ferrocenium/ferrocene redox couple and hence they are often designated as 'non-fullerene acceptor' in OSCs. ${ }^{18}$ The HOMO-LUMO energy gap of such materials, a prerequisite parameter to find a befitting organic electronic application, can be satisfactorily tuned by incorporating donor/acceptor moieties on perylene core, as reported in multitudinous literature. It is pleasing to note that PDI is very effectual in generating an ambient-stable radical anion in solution because of its outstanding electron-deficient nature. ${ }^{19}$ Where the generation and isolation of ultrastable radical anion is a strenuous task, PDIs there provide a golden opportunity to solve this predicament. Based on aforementioned appreciable photophysical, thermal and photochemical properties, both PDI and PMI have encompassed their applications in various spheres such as fluorescence sensing, bio-imaging, single molecular spectroscopy, organic photocatalysis, supramolecular assemblies, hostguest interaction and light-matter interaction in organic photonics (see Figure 2). It should be noted that making of water-soluble PDI/PMI derivatives for biological and medicinal applications is very tedious, nevertheless, tireless effort of few research groups has shown the ray of hope in this regard by introducing ionic/hydrophilic groups on ortho-/ bay-/imide-positions. ${ }^{20}$ Moreover, their high electron-accepting character has been effectively utilized in organic semiconductors, organic field-effect transistors (OFETs) and

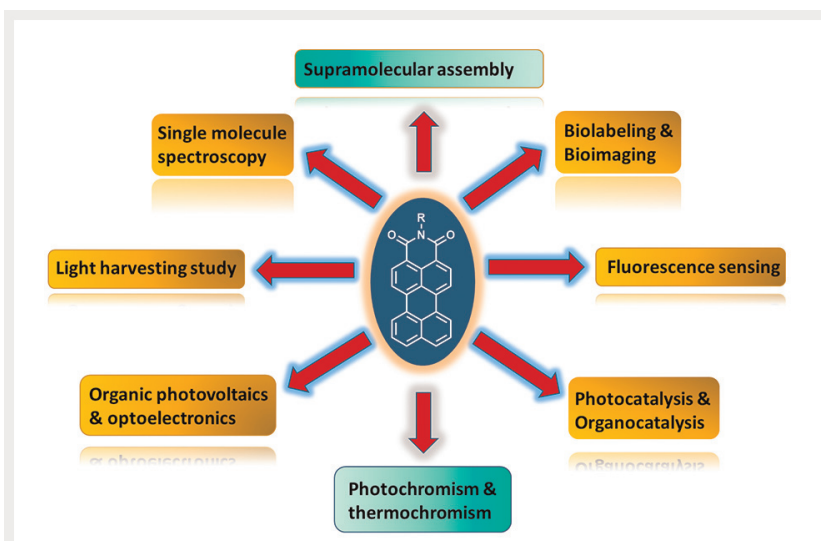

Figure 2 Representation of various applications of PMI derivatives.

organic photovoltaics (photodetectors, bulk-heterojunction solar cells and dye-sensitized solar cells, DSSCs). In spite of countless literatures on PDI/PMI-based OFETs and OSCs, we can find only a few reports on perylene-imide-based organic light-emitting diodes. Till date, PDI derivatives have been immensely explored in organic materials arena, but needless to say, in recent times, its sister analogue PMI has also contributed along similar lines. Hence, we would like to provide a comprehensive review by highlighting the most important development of PMI chemistry focusing on the synthesis of numerous PMI derivatives, their photophysical, electrochemical, self-assembling properties, and their diversified applications.

\section{Advantages of PMI over PDI}

In the small family of perylene, PMI holds some unique properties over its symmetric analogue PDI although they share a number of common properties. Firstly, PMI possesses an inherent higher ground-state dipole moment (6.4 D) along the long-conjugated axis compared to PDI. Hence, the self-assembled nanostructures created by PMI derivatives experience a profound dipolar nature in bulk, which impacts their energy transfer process as unravelled by Wasielewski's group. Secondly, the asymmetrically positioned imide moiety in the parent PMI scaffold furnishes intermolecular charge transfer (ICT) characteristics on being conjugated with other moieties. In 2007, Mullen and coworkers ensconced this concept by synthesizing donor-appended PMI derivatives where the extent of ICT characteristics by different donors influenced the photoelectrochemical properties along with solar cell efficiency in DSSCs. ${ }^{21}$ PMI also fluoresces mostly in the bathochromically shifted region relative to parent PDI's emission region, therefore Stokes shift of PMIs are comparatively larger than those of PDIs. The Stokes shift of PMI can be extremely enlarged by effective in- 
troduction of strong donors in the peri-position as established by Marks et al. They synthesized a series of PMI-based donor (D)-acceptor (A) derivatives (donors: indolizine, carbazole, diphenylamine, dialkylamine and dibenzazepine) possessing more than $100 \mathrm{~nm}$ stokes shifts which were utilized as suitable candidates in luminescent solar cell concentrator devices. ${ }^{22}$ They are comparatively more photostable than their PDI counterpart. From the electrochemical point of view, $0.4 \mathrm{~V}$ more negative one-electron reduction potential makes them more advantageous in energy transfer processes along with the ability to achieve greater open-circuit voltage $\left(V_{\mathrm{OC}}\right)$ in OSC devices. Synthetically, PMI is recognized as an important intermediate to prepare higher rylene imides and their derivatives for desired applications due to the availability of a peri-position for functionalization as compared to their diimide congener.

\section{Challenges in Working with PMI and Ways to Overcome}

Although PMI possesses several advantageous properties, the inherent poor solubility in common solvents is the major bottleneck in working with PMI. The highly planar and hydrophobic surface makes it an inherently poorly soluble organic dye. Therefore, overcoming its poor solubility is a synthetically challenging task. Thanks to the seminal effort of synthetic chemists who have discovered various synthetic routes to overcome this challenge i.e., introducing sterically hindered and bulky substituents in -imide/-ortho/-bay positions of the PMI core. Introduction of bulky and branched chain amines in the imide position only obstructs their intense $\pi-\pi$ stacking without hampering their optical properties. The ground- and excited-state optical properties are not influenced by $N$-imide substitutions because of the presence of node on imide nitrogen in both HOMO and LUMO. Ortho-substitution also plays a similar role in increasing solubility. Conversely, bay-substituents such as bulky and voluminous alkoxy and aryloxy groups significantly alter PMIs' photophysical properties in addition to improvement in their solubility in common organic solvents. On the other hand, the most sensitive peri-position which contributes very little in solubility enhancement is only responsible for fine-tailoring of photophysical and electrochemical properties of PMI in solution (vide infra). One more disadvantage borne by PMI is that the parent molecule is very susceptible to aggregate in solid state because of their strong intermolecular interactions. In this regard, literature reports reveal incorporation of long hydrophobic branch chains on the $\mathrm{N}$ imide region sterically blocks their self-aggregation which effectively gives rise to their solid-state emissive property.

\section{Various Aspects of Reactivity of Different Positions}

Both PMI and PDI dyes share common reactive positions such as imide, ortho- and bay- on their core, fortunately PMIs open an extra and highly reactive position i.e., peri- position to the synthetic chemist. The reactivity and type of reactions on these abovementioned positions are different as reported in the literature. The functionalization on imide position by amine-containing long/branched alkyl chains or sterically hindered phenyl groups tremendously increases the solubility of aggregation-prone PMI derivatives. Orthofunctionalization by branched alkyl chains sterically obstructs the strong $\pi-\pi$ interaction of PMIs and PDIs. ${ }^{7 d}$ The less reactive ortho-positions also help to introduce small electron-withdrawing/electron-donating groups on the perylene core. Bay-positions of PMI core are comparatively more reactive than imide- and ortho-positions which mostly favour nucleophilic substitutions with bulky/spacious groups to increase the solution processability. Additionally, this position also offers traditional coupling reactions and bay-annulation to make them as donor-acceptor conjugated chromophores. The distinct peri-position propounds the nucleophilic substitution as well as introduction of donor/acceptor moieties via coupling and annulation method. The peri-position impressively tailors the photophysical properties of PMIs rather than effecting on increasing solution processability, which has caught an exceptional attention of researchers to explore this position more.

\section{Synthesis of the PMI core}

The synthetic chemistry of dyes based on PMIs has been developed very rapidly over the past few years. The synthesis of PMI was first started by Langhals' group, who developed a method to synthesize different derivatives of PMIs. ${ }^{23}$ According to them, when commercially available 3,4,9,10perylenetetracarboxylic dianhydride (PTCDA) was treated with a primary amine in the presence of zinc acetate and molten imidazole at high temperature and pressure, PMI was obtained. The side-products of this reaction were found to be PDI and perylene (as shown in Figure 3). The major challenge in working with PMIs is to overcome their intrinsically low solubility. A widespread approach to improve their solubility has been achieved by introducing sterically demanding residues at the imide positions that minimize the strong aggregation tendency. Especially, ortho-substituted anilines (2,5-di-tert-butyl aniline, 2,6-diisopropyl aniline, etc.) and branched amines (2-ethylhexylamine, etc.) are excellent aggregation blockers that produce PMIs with good solubility in organic solvents. 


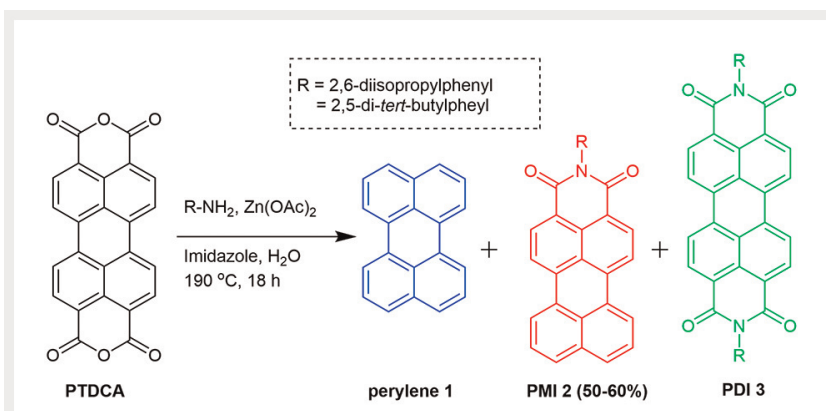

Figure 3 The synthetic pathway of PMI first reported by Langhals' group. ${ }^{23 a}$

\section{Synthesis of PMI Derivatives}

Although the synthetic method for making PMIs given by Langhals' group is the most popular and widely accepted, but it is still very challenging to efficiently prepare functional PMI-based materials on a large scale. Another disadvantage of their method is that it can be used in the preparation of only a few $\mathrm{N}$-aryl-substituted PMIs, because only few aromatic amines can tolerate such harsh reaction conditions (high temperature and long reaction time in a pressure autoclave). Recently, Xiao's group proposed a new and efficient approach for synthesizing PMIs with different primary amines including aromatic, branched chain or long-tail at the imide position (as shown in Figure 4). ${ }^{24}$

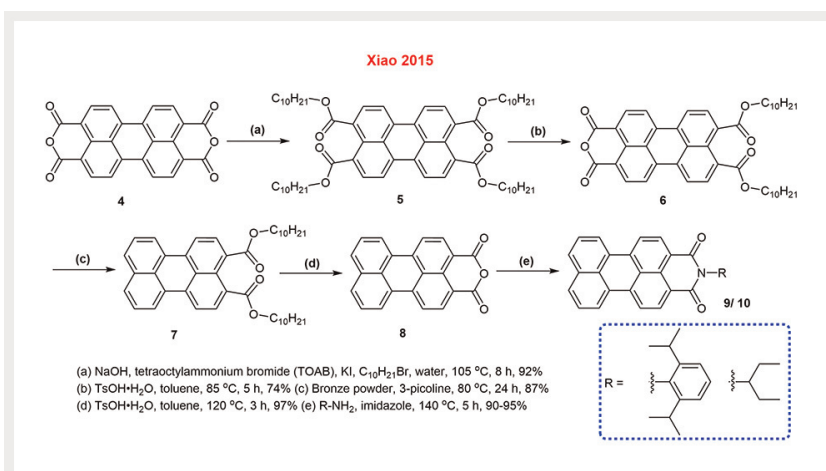

Figure 4 Another synthetic strategy of PMI as reported by Xiao's group. ${ }^{24}$

First, they chose $\mathrm{C}_{10} \mathrm{H}_{21}$-based alkyl bromide as the functional groups because of the excellent solubility provided by this group during intermediate stages of processing. After esterification, decarboxylation and hydrolysis, the final product 3,4-perylenedicarboxylic monoanhydride (8) was obtained as insoluble brown solid. Subsequently, 8 was treated with different amines in imidazole at $140^{\circ} \mathrm{C}$ for $5 \mathrm{~h}$ affording PMIs in quantitative yield. This method of PMI syn- thesis has some advantages (such as wide ranges of amine choice, mild reaction conditions and high yield) over conventional synthetic pathways and could therefore be applied for the large-scale synthesis. ${ }^{24}$

\subsection{Bromination of PMI}

Efficient halogenation (especially bromination) of the perylene core in PMIs affords the building blocks for further functionalization of PMIs required for desired applications. Selective bromination yielding mono-brominated PMI 11 or tri-brominated PMI 12/13 along with tetra-brominated PMI 14 is possible by adjusting the solvent, bromine equivalents, and reaction temperature conditions (see Figure 5). Müllen's group has developed a synthetic method for the mono- and tri-bromination of PMIs. ${ }^{25}$ However, a facile synthetic method for making tetra-brominated PMI 14 has still not been successfully developed because it gives other regioisomers of tri-brominated PMI $(\mathbf{1 2}, \mathbf{1 3})$ along with tetra-brominated PMI 14. Mostly, compound $\mathbf{1 4}$ is isolated only after tedious repetitive precipitations with a very low yield. ${ }^{26}$ Later, our group developed a synthetic protocol for the facile synthesis of tetra-brominated PMI with a relatively simple workup procedure. ${ }^{27}$ In this protocol, we treated PMI 2 with 200 equivalents of bromine which affords 1,6,9,10-tetra-bromo PMI in $46 \%$ yield (as shown in Figure 5). Recently, Peneva's group reported a synthetic procedure in which they have reported the synthesis of 1,6,9,10- and 1,7,9,10-tetrabromoperylene-3,4-dibutylester in three steps from perylene $3,4,9,10$-dianhydride (4). ${ }^{28}$

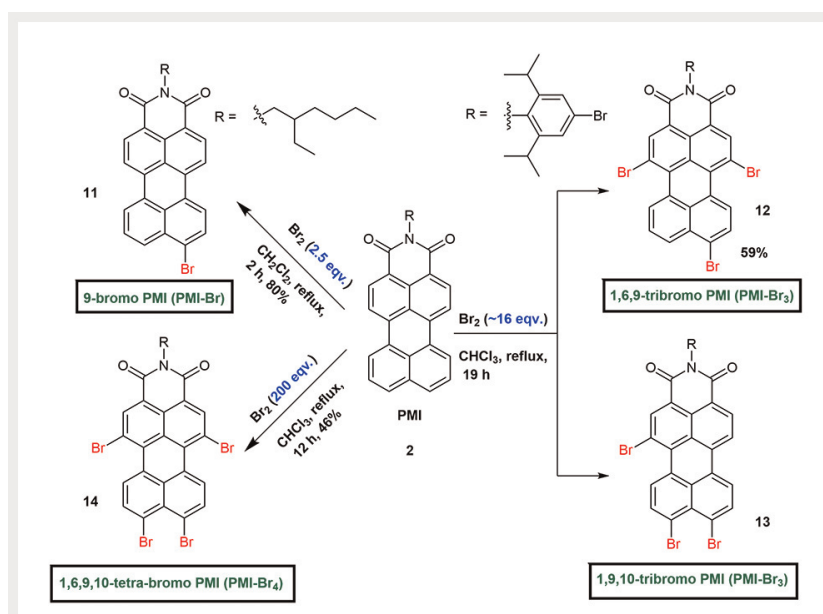

Figure 5 Synthesis of mono-, tri- and tetra-bromo PMI derivatives. 


\subsection{Synthesis of PMI Derivatives Using Coupling Reactions}

The brominated PMIs serve as building blocks for various palladium-catalyzed cross-coupling reactions, such as Suzuki, Sonogashira or Buchwald-Hartwig cross-coupling, leading to diverse PMI derivatives.

\subsubsection{Suzuki Coupling}

Mono-bromo PMI (PMI-Br) can be used to introduce different substituent groups at the peri-position via the palladium-catalyzed Suzuki cross-coupling reaction. The library of compounds including electron donating and polyaromatic group functionalized at the peri-position of PMI were synthesized in good to excellent yield via two possible routes of Suzuki cross-coupling (as shown in Figure 6). ${ }^{29}$ In route 1, the mono-brominated PMI 11 was coupled with different boronic acid derivatives under Suzuki-Miyaura coupling conditions affording corresponding electron-donating and polyaromatic PMI derivatives 16-22. On the other hand, in route 2, the synthesis of phenanthrene-conjugated PMI 23 was carried out in a slightly different way. Firstly, the mono-brominated PMI $\mathbf{1 1}$ was converted to functionalized boronic ester PMI-BE (15) followed by coupling with 9-bromo-phenanthrene using the Suzuki-Miyaura coupling protocol. ${ }^{29}$ The two PMIs at the peri-position can be coupled to each other via a linker (such as fluorine, silafluorene and carbazole) using the Suzuki-Miyaura cross coupling reaction (as shown in Figure 7). ${ }^{30}$

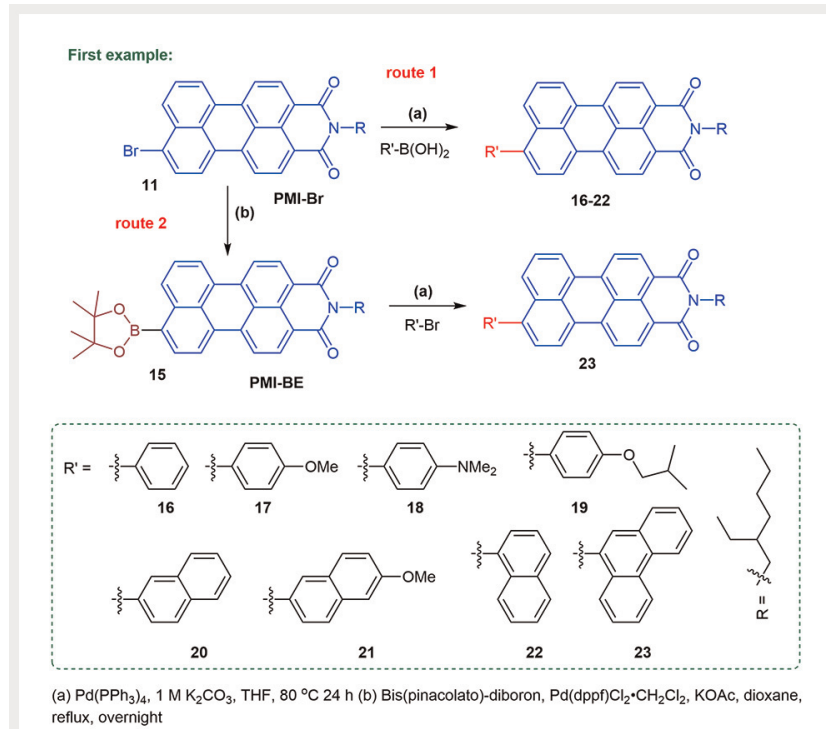

Figure 6 Suzuki coupling reactions of PMI.

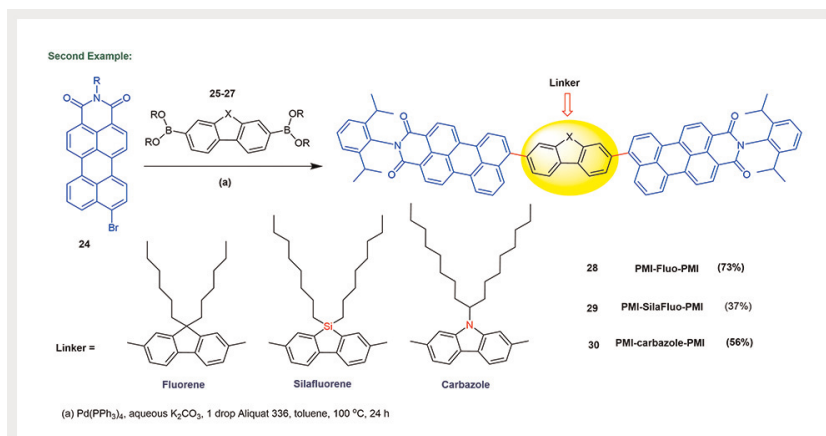

Figure 7 Synthesis of linker-coupled PMI derivatives via the Suzukicoupling reaction.

\subsubsection{Sonogashira Coupling}

To introduce the alkyne group at the peri-position (9-position) of PMI, PMI-Br (11) was treated with triisopropylsilyl acetylene (TIPS acetylene) in the presence of tris(dibenzylideneacetone)dipalladium $(0)\left[\mathrm{Pd}_{2}(\mathrm{dba})_{3}\right]$, tris $(o$-tolyl)phosphine $\left[\mathrm{P}(o \text {-tolyl })_{3}\right]$ and trimethylamine $\left(\mathrm{Et}_{3} \mathrm{~N}\right)$ and followed by deprotection of TIPS acetylene to get PMI-acetylene (32). This PMI-acetylene $\mathbf{3 2}$ can be utilized for the further introduction of several different groups through alkyne. For instance, 32 was coupled with the 9-bromo-phenanthrene using Sonogashira-Hagihara cross-coupling reaction conditions to get $\mathbf{3 4}$ in which the PMI core is appended with a rigid alkyne-phenanthrene moiety (as shown in Figure 8, top part). ${ }^{31}$ Our group has also developed microwave-assisted Sonogashira coupling to make new PMI derivatives that

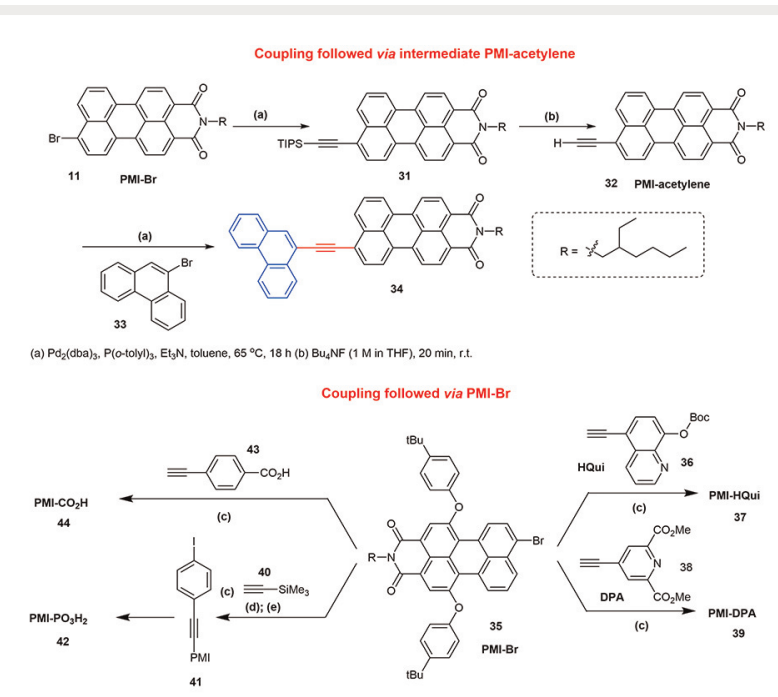

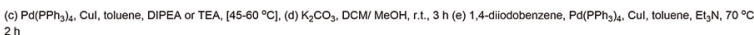

Figure 8 Representation of Sonogashira-Hagihara coupling on PMI derivatives. 
helped to achieve higher yield of the compounds in less time compared with the traditional coupling method. ${ }^{32}$

Similarly, PMI-Br (35) in which the bay-positions (1,6position) are functionalized with 2,5-di-tert-butylphenoxy groups while the bromo at the peri-position (9-position) of 35 is coupled with a series of groups using the Sonogashira-Hagihara cross coupling reaction protocol afforded 37, 39, 42 and 44 (as shown in Figure 8, bottom part). ${ }^{11 b}$ Phenoxylation at the bay-position of halogenated PMIs is one of the most common ways to increase the solubility of perylene dyes. This increase in solubility mainly results from a twisting in the perylene core that reduces its tendency towards $\pi-\pi$ stacking. Besides increasing solubility, the photophysical properties of phenoxylated PMIs are also immensely modulated on bay-functionalization with phenoxy substituents.

\subsubsection{Buchwald-Hartwig Coupling Reaction}

To introduce the $\mathrm{C}-\mathrm{N}$ bond in PMI at the peri-position, the Buchwald-Hartwig cross coupling reaction was carried out. In this coupling reaction, mono-brominated PMI 24 was coupled with 45, 46, and 47 in the presence of $\operatorname{Pd}_{2}(\mathrm{dba})_{3}$, tri-tert-butylphosphine and sodium tert-butoxide $\left(\mathrm{NaO}{ }^{t} \mathrm{Bu}\right)$ in anhydrous toluene to get the coupled products 48,49 and 50, respectively (as shown in Figure 9, top part). ${ }^{33}$ In the second method under Buchwald-Hartwig coupling reaction conditions, $\mathbf{5 1}$ was coupled with a series of different al$\mathrm{kyl}$ amine derivatives in the presence of $\mathrm{Pd}_{2}(\mathrm{dba})_{3}$, BINAP (2,2'-bis(diphenylphosphino)-1,1'-binaphthyl) and cesium

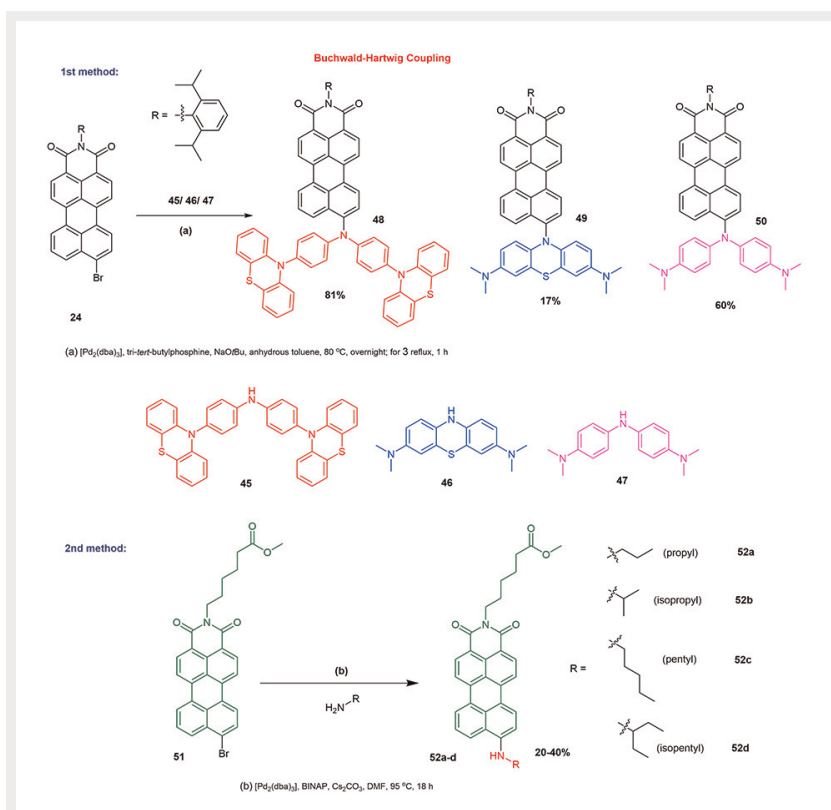

Figure 9 Synthesis of new PMI derivatives by the Buchwald-coupling reaction. carbonate $\left(\mathrm{Cs}_{2} \mathrm{CO}_{3}\right)$ in DMF to afford $\mathbf{5 2}(\mathbf{a}-\mathbf{d})$ (see Figure 9, bottom part). ${ }^{34}$

\subsection{Nucleophilic Substitution Reactions}

Brominated PMIs are also very important building blocks for additional functionalization by performing different sets of reactions to get various PMI derivatives. The nucleophilic substitution reaction is one of them in which the bromine atom from PMI can be replaced by a series of groups. When tetra-brominated PMI 14 was treated with 4-tert-octylphenol, thiophenol and 3-hydroxypyridine in the presence of a base, all the four bromine atoms were replaced by their corresponding nucleophiles to get $\mathbf{5 3}, \mathbf{5 4}$ and $\mathbf{5 6}$ in good to excellent yields (57\%, $86 \%$ and $82 \%$, respectively; as shown in Figure 10). ${ }^{27}$

Previous studies bring forth the conception that bromine atoms at the peri-position of PMI exhibits different reactivities than the ones at the bay-position, ${ }^{33}$ Validating this proposition, tetra-brominated PMI 14 upon reaction with piperidine at $55^{\circ} \mathrm{C}$ for $24 \mathrm{~h}$ gives regioselective peri-substituted PMI 55 with both the bromine atoms at the bay-position remaining inactive (as shown in Figure 10, (c) pathway). ${ }^{27}$

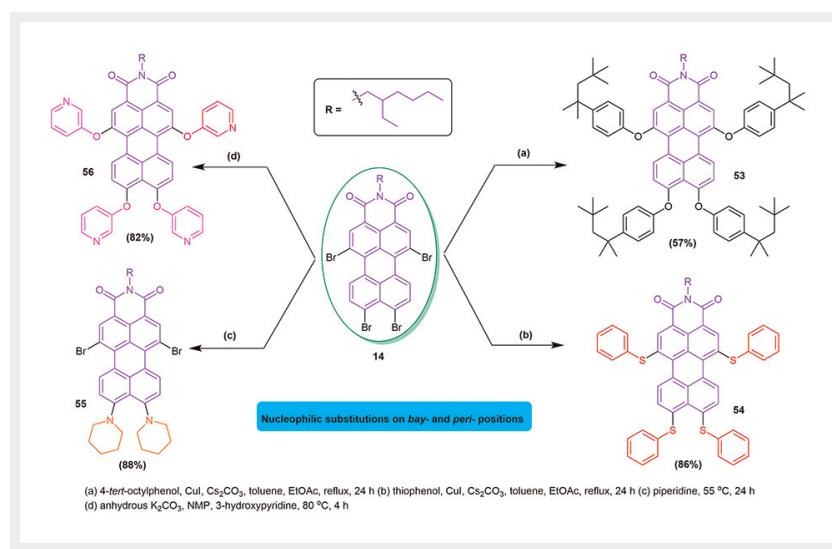

Figure 10 Nucleophilic substitution reaction on tetra-bromo-2-ethyl-1hexyl PMI.

\subsection{Peri-Annulation Reaction}

Peri-annulation of PMI can be considered as a promising and alternative strategy to introduce donor-acceptor character in PMI derivatives other than traditional Suzuki/Sonogashira coupling, as revealed by Müllen and co-workers. They achieved a 6-membered aniline-annulated tetrachloro PMI derivative in remarkable yield, but the yield was moderate for another 7-membered compound upon using 1,2-diami- 


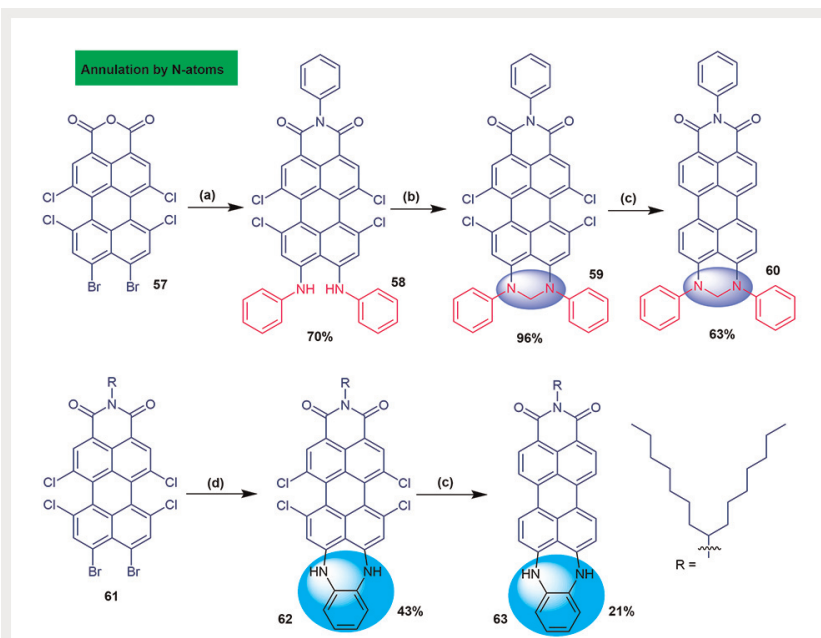

(a) Aniline, $180^{\circ} \mathrm{C}, 5 \mathrm{~h}$ (b) $\mathrm{HCHO}, \mathrm{TFA}^{\circ} \mathrm{CHCl}_{3}$, reflux, $1.5 \mathrm{~h}$ (c) $\mathrm{KOH}$, Ethylene glycol, $165^{\circ} \mathrm{C}, 4 \mathrm{~h}$ (d) 1,2-diaminobenzene (a) Aniline, $180^{\circ} \mathrm{C}, 5 \mathrm{~h}$
NMP. $180^{\circ} \mathrm{C}, 5 \mathrm{~h}$

Figure 11 Peri-annulation reaction of PMI by N-containing moieties.

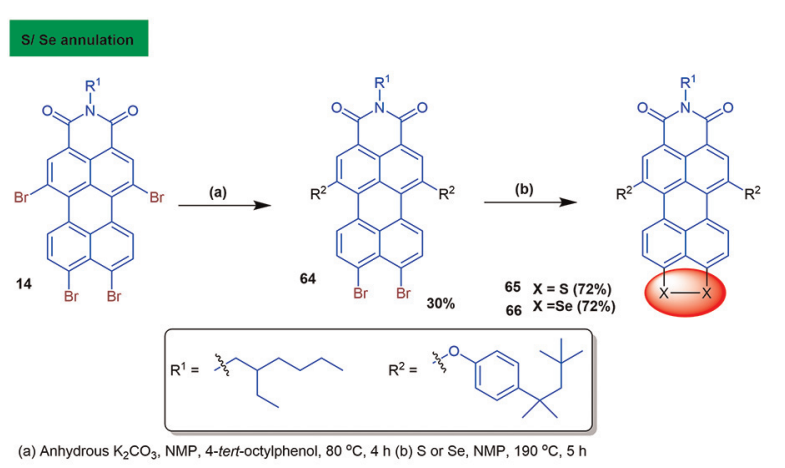

Figure 12 Synthesis of S/Se annulated bay-functionalized PMI compounds.

nobenzene as an annulating reagent (as shown in Figure 11). ${ }^{35}$

These two derivatives possessing synergistic push-pull effect were the first members of the peri-annulated PMI family. Inspired by this strategy shown by them, our group also carried out $\mathrm{S} / \mathrm{Se}$ annulation reaction in the peri-position of substituted-PMI and was successful in achieving good yield (as shown in Figure 12). ${ }^{27}$

The S/Se annulated compounds exhibited drastic change of visible color along with extension of absorption characteristics due to the ICT effect. Wang's group reported a new PMI-coronene hybrid material by ring fusion and acquired an appreciable yield (as shown in Figure 13). ${ }^{36}$ Recently, the same group has also successfully added new PMI-fullereneannulated compounds abbreviated as PMI-O-Fuller and PMI-S-Fuller in this small family. For synthesizing this com-

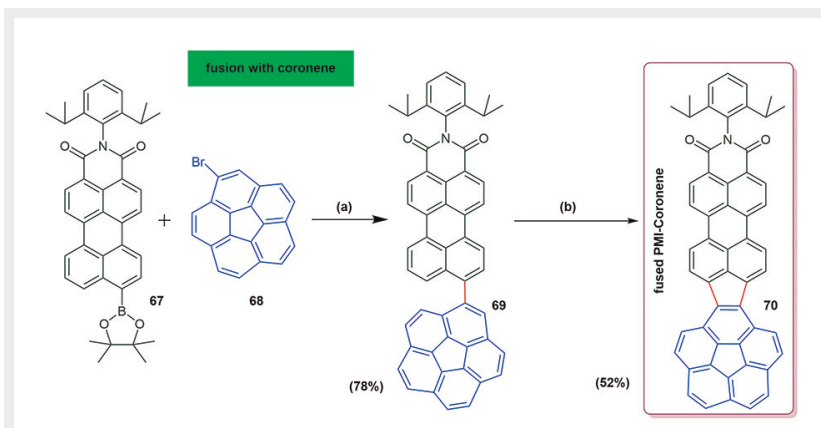

(a) $\mathrm{Pd}\left(\mathrm{PPh}_{3}\right)_{4}, \mathrm{~K}_{2} \mathrm{CO}_{3} / \mathrm{H}_{2} \mathrm{O}$, toluene/ ethanol, $90^{\circ} \mathrm{C}, 12 \mathrm{~h}$ (b) $\mathrm{PIFA}, \mathrm{BF} \cdot \mathrm{Et}_{2} \mathrm{O},-40^{\circ} \mathrm{C}, 2 \mathrm{~h}$

Figure 13 Synthesis of fused PMI-coronene derivative via Suzuki coupling.

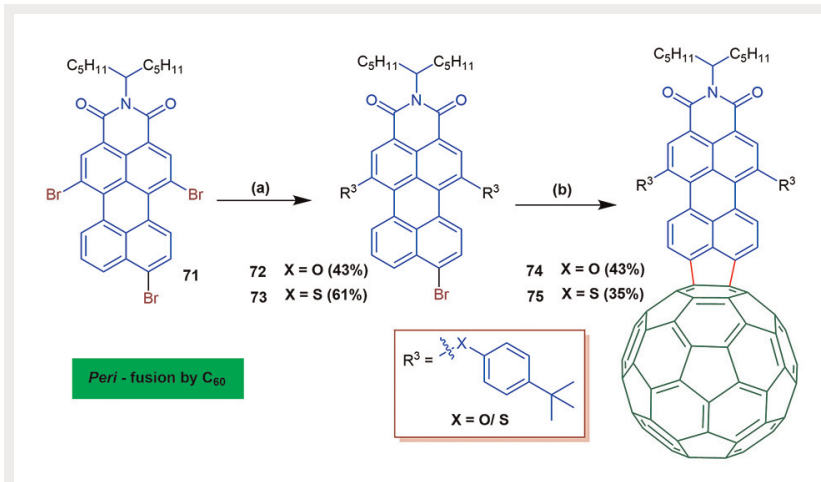

(a) Anhydrous $\mathrm{K}_{2} \mathrm{CO}_{3}, \mathrm{NMP}, 80^{\circ} \mathrm{C}, 12 \mathrm{~h}$ (b) $\mathrm{Pd}(\mathrm{OAc})_{2}, \mathrm{PCy}_{3} \cdot \mathrm{HBF}_{4}, \mathrm{~K}_{2} \mathrm{CO}_{3}, 1-\mathrm{MeNp}, \mathrm{C}_{60}, 200^{\circ} \mathrm{C}, 3 \mathrm{~h}$

Figure 14 Synthetic pathway of fused PMI- $\mathrm{C}_{60}$ hybrids.

pound, they performed a cyclization reaction between $\mathrm{PMI}-\mathrm{Br}$ and $\mathrm{C}_{60}$ in presence of $\mathrm{Pd}^{2+}$ catalyst and $\mathrm{PC}_{3}$ ligand and obtained considerable yield of $\sim 40 \%$ (as shown in Figure 14). ${ }^{37}$ The authors have demonstrated that this hybrid material can be used as a potential candidate for organic photovoltaics.

It is very familiar that the PMI core is comprised of only carbon and hydrogen atoms. How will it be if the carbon atom in the PMI core is substituted by other heteroatoms? Incentivized by this, Chen's group in 2020 has developed a novel N-doped PMI derivative exhibiting the push-pull effect. The compound was prepared via a one-pot reaction; first Suzuki-coupling reaction between 4,5-dibromonaphthalene and 5-pinacolatoboryl-8-methoxyquinoline followed by intermolecular cyclization reaction (as shown in Figure 15). ${ }^{38}$ This may be called as a convenient and facile approach that opens the door for constructing other heteroatom-doped PMI derivatives in future. The most important building block for making higher rylene imides is perylene3,4-dicarboximide (PMI) which was obtained from readily available PTDCA by following a previously reported protocol 


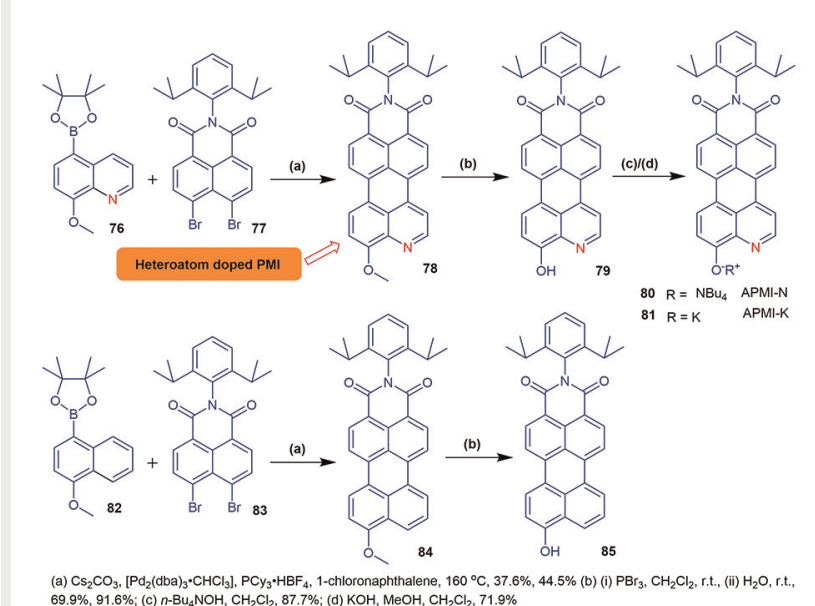

(a) $\mathrm{Cs}_{2} \mathrm{CO}_{3},\left[\mathrm{Pd}_{2}\left(\mathrm{Cba}_{3}\right) \cdot \mathrm{CHCC}_{3}\right], \mathrm{PC}_{3} \cdot \mathrm{HBF}_{4}, 1$-chloronaphthalene, $160^{\circ} \mathrm{C}, 37.0 \%$

Figure 15 Synthesis of N-doped push-pull PMI and non-doped PMI derivatives.

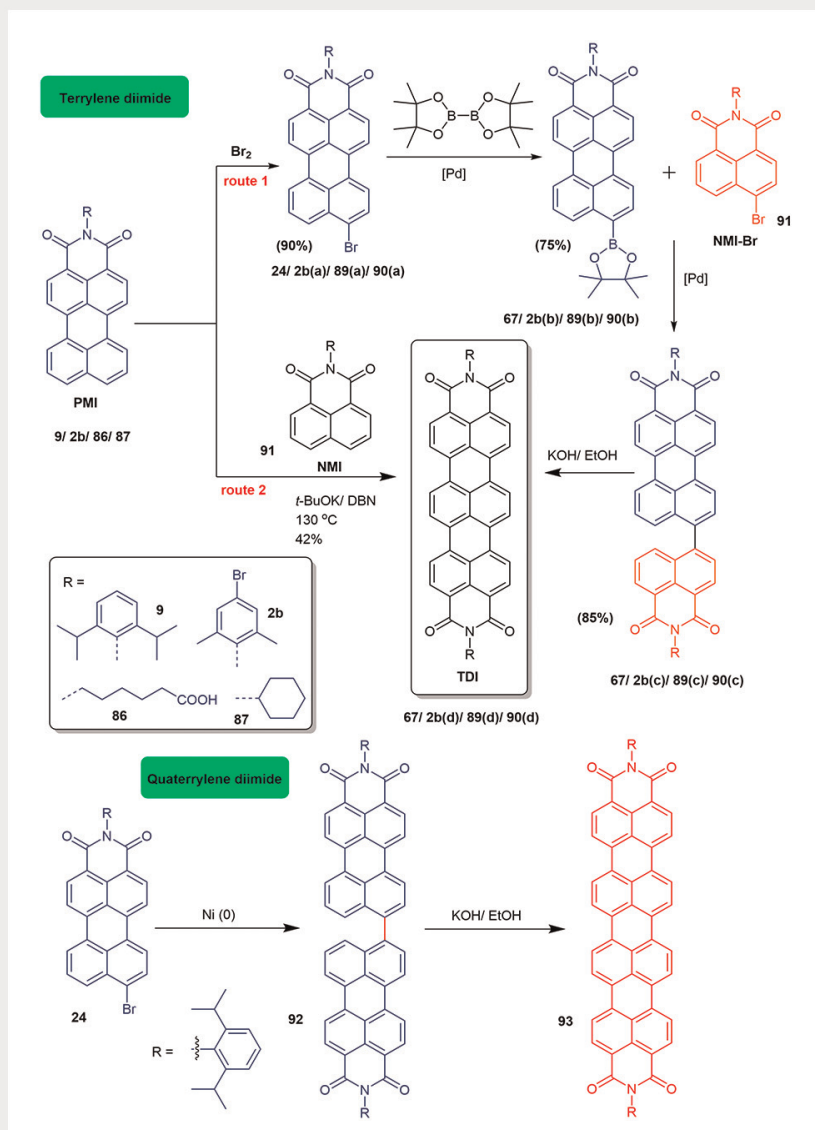

Figure 16 Synthetic routes for preparation of TDI and QDI.

(see Figure 3). ${ }^{23}$ The first two higher homologues in the family of rylene diimides, i.e., TDI and QDI, were synthesized in

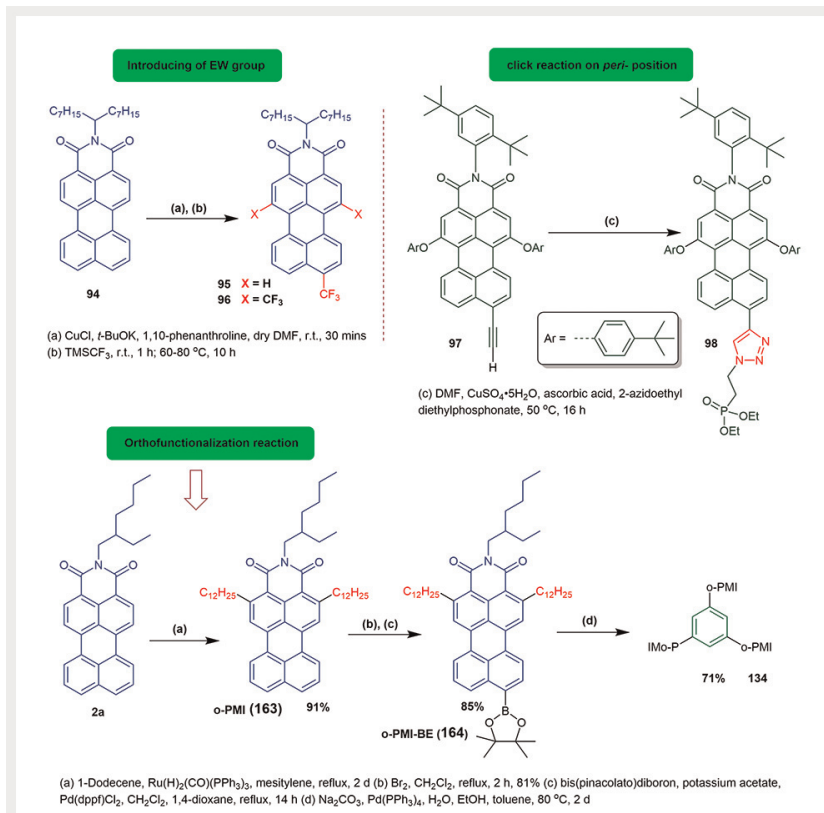

Figure 17 Synthetic pathway for introduction of an electron-withdrawing group in the PMI core (left), representation of an azide-click reaction on the peri-position of PMI (right), and an ortho-functionalization reaction of PMI (bottom).

two possible routes from PMI and naphthalene monoimide (NMI) (see Figure 16, top part). In route 1, PMI was first mono-brominated to get PMI-Br followed by its conversion to PMI-boronic ester which was further coupled with NMI-Br via palladium-catalyzed Suzuki cross-coupling reaction. This was subsequently followed by cyclo-dehydrogenation affording TDI. Alternatively, in route 2, TDI was obtained by direct coupling and fusion of PMI with NMI in presence of DBN and $t$-BuOK in a 'one-pot' reaction. ${ }^{3}$ Similarly, QDI 93 was synthesized by a two-step reaction via homo-coupling of PMI bromide $\mathbf{2 4}$ to get $\mathbf{9 2}$ followed by cyclohydrogenation under basic conditions (see Figure 16, bottom part). ${ }^{39}$

Till now, the above discussion on PMI's reaction has been emphasized on incorporation of donor or other moieties by coupling or annulation reactions. In 2014, Wasielewski's group unveiled a synthetic strategy to introduce electronwithdrawing $-\mathrm{CF}_{3}$ groups on both bay- and peri-positions (Figure 17, top-left part) which triggered a radical-anion generation process. ${ }^{40}$ They carried out this one-step perfluorination reaction in the presence of catalytic amount of $\mathrm{CF}_{3} \mathrm{TMS}$ (Ruppert-Prakash reagent) and $\mathrm{Cu}(\mathrm{I})$ to procure appreciable yield. A few years ago, Harriman's group established the popular azide-alkyne click reaction in a PMI system using $\mathrm{Cu}(\mathrm{II})$ and ascorbic acid as coupling agents (Figure 17, top-right part). ${ }^{41}$ This can be assumed that coupling on such a photoactive material can remarkably tune the optical 


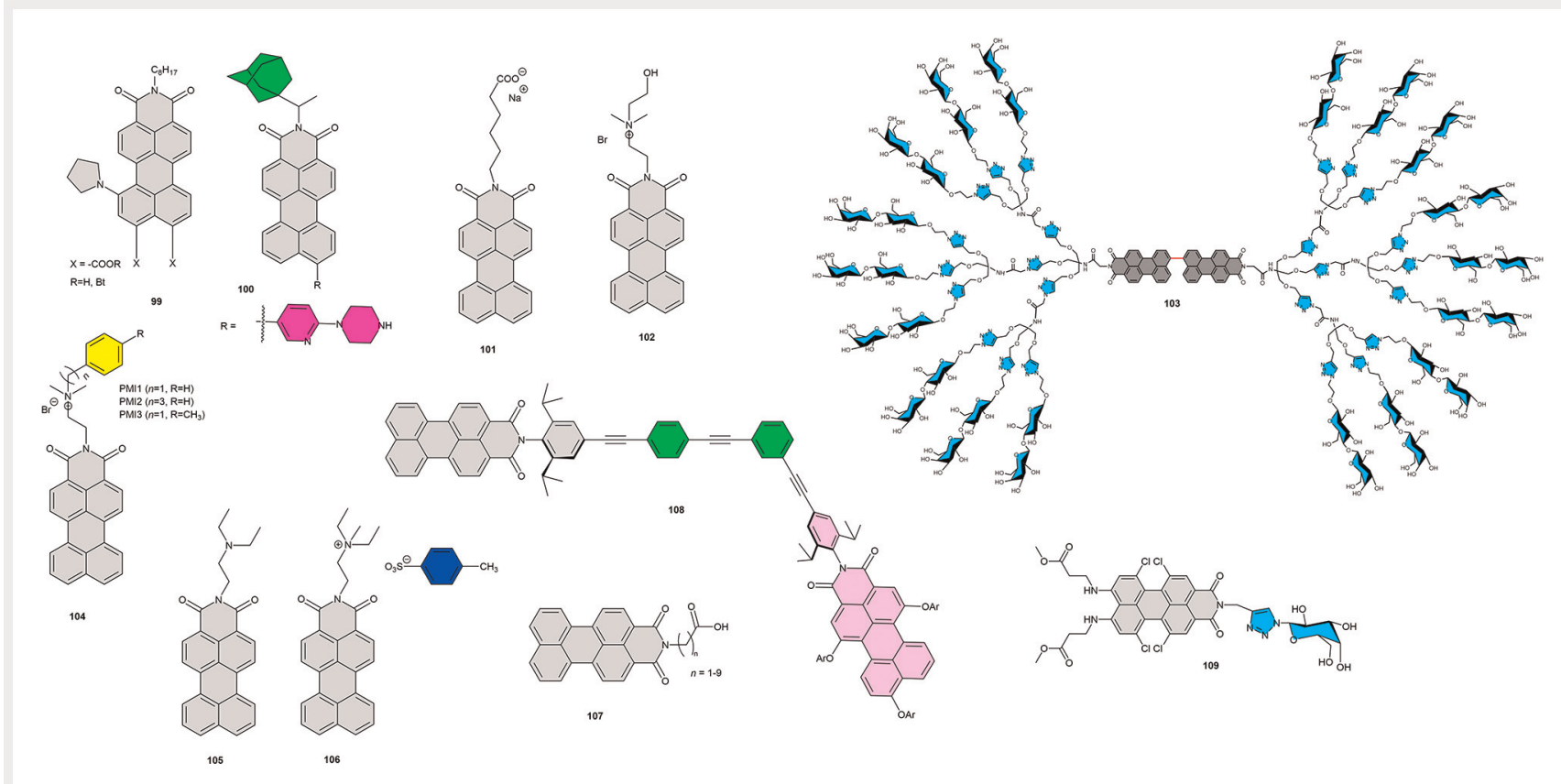

Figure 18 Examples of imide-functionalized PMIs.

properties of the whole compound. In the bottom part of Figure 17, the ortho-functionalization reaction on PMI is represented, as evolved by Wasielewski and co-workers. ${ }^{42}$ In this reaction, at first, the less reactive ortho-position of PMI was activated in the presence of $\mathrm{Ru}(\mathrm{II})$-catalyst, followed by addition of an alkene chain with Ru-PMI complex to finally yield an alkyl-substituted ortho-functionalized PMI derivative (164).

The structures of other PMI derivatives discussed in this review are provided in Figures 18-20.

\section{Photophysical Properties of PMI}

The basic structural core of rylene is favorable for the creation of functional dyes due to their synthetic accessibility along with their outstanding photophysical properties. ${ }^{4 a, 23 a}$ The extraordinary chemical, thermal, and photochemical stabilities are the outcome of their low-lying LUMO energy level. ${ }^{4 a}$ The introduction of additional naphthalene units into the conjugated core only results in minor changes in the LUMO energy level, while the HOMO rises considerably. The most attractive feature of rylene imides is their optical properties with an allowed transition which results in strong vibronically structured absorptions. ${ }^{3}$ The unsubstituted PDIs and PMIs absorb in the visible region between 400 and $550 \mathrm{~nm}$ with high molar extinction coefficients in the order of $10^{4} \cdot \mathrm{M}^{-1} \cdot \mathrm{cm}^{-1}$. The extension of the conjugated core by successive introduction of naphthalene units causes an increase in their absorption coefficient coupled with a linear dependence in the absorption maxima accompanied with a bathochromic shift of $\sim 100 \mathrm{~nm}^{3}$ PMIs and PDIs have outstanding near-unity fluorescence QY. ${ }^{43}$ Their emission spectrum typically exhibits a small Stokes shift and appears as a mirror image of the absorption profile. The absorption and fluorescence properties of unsubstituted PDIs and PMIs are devoid of any solvent dependence. The outstanding photostability combined with the high brightness makes PDI and PMI ideal probes for single-molecule spectroscopy (SMS) investigations. ${ }^{44}$ Generally, substitution at the imide position does not have any influence on the optical properties of PMIs (see Table 1, entries 3 and 4) and PDIs (entries 5 and 6). Only a bay-substituent at the perylene core does not alter much their optical properties but significantly influences the solubility of PMIs and PDIs. However, a judicious substitution of the donor group at the peri-position of PMI can generate the push-pull character which significantly modulates the absorption and emission properties. Literature data shows that introduction of 4-tert-butylphenoxy group at the peri position of PMI shifts its absorption maximum to the longer wavelength region by $28 \mathrm{~nm}$ in comparison to peri-unsubstituted PMI. On the other hand, with the introduction of three 4-tert-butylphenoxy group at 1, 6, and 9-positions of the PMI [PMI(OAr) $)_{3}$, entry 8], the absorption maximum shifted to the red region by $32 \mathrm{~nm}$. This observation suggests that there are small effects on the absorption maximum when both the bay regions (C-1 and C6) of PMI were substituted. However, when the peri-position 


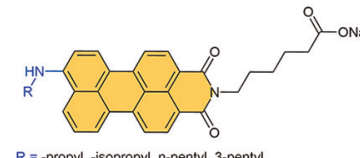

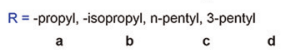
116 (a-d)

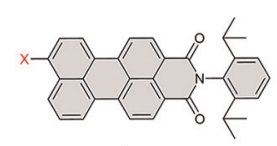

(a-c)
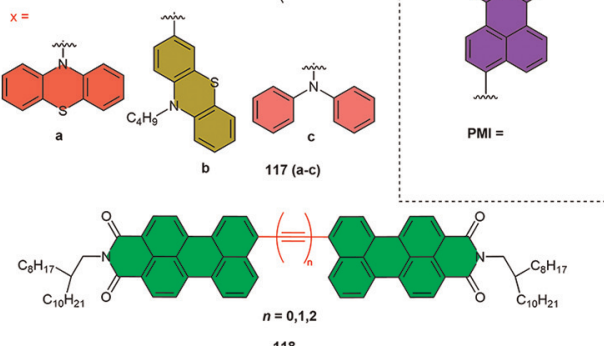

$\mathrm{PMI}=$

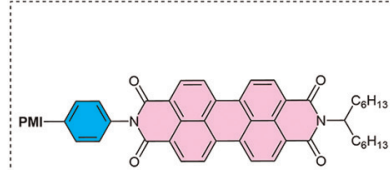

PMI

110
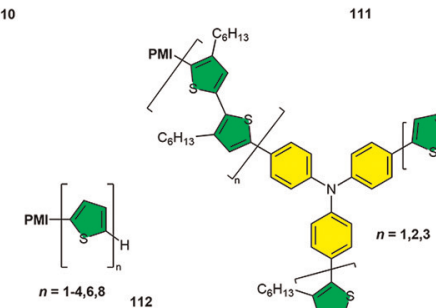

$\left.{ }^{C_{6} \mathrm{H}_{13}}\right]_{\mathrm{PMI}}$
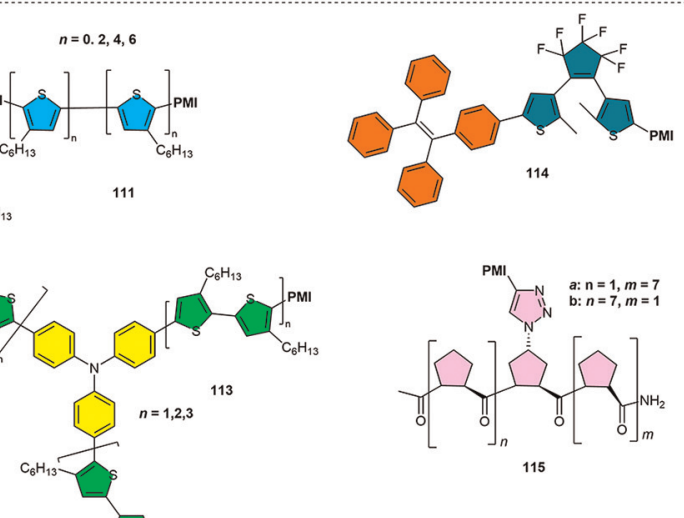

(a-b) $=x$
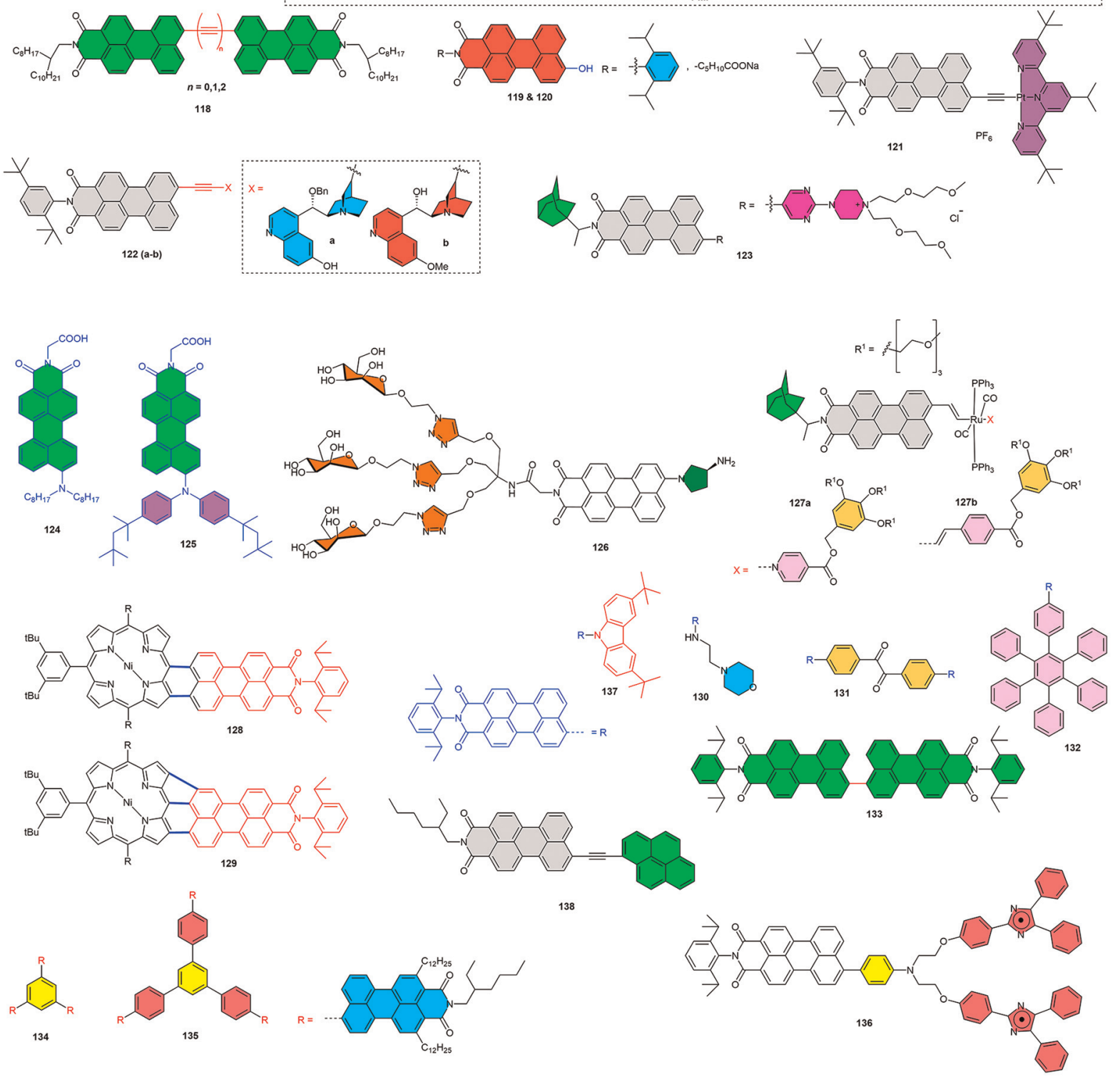

Figure 19 Examples of peri-functionalized PMIs. 


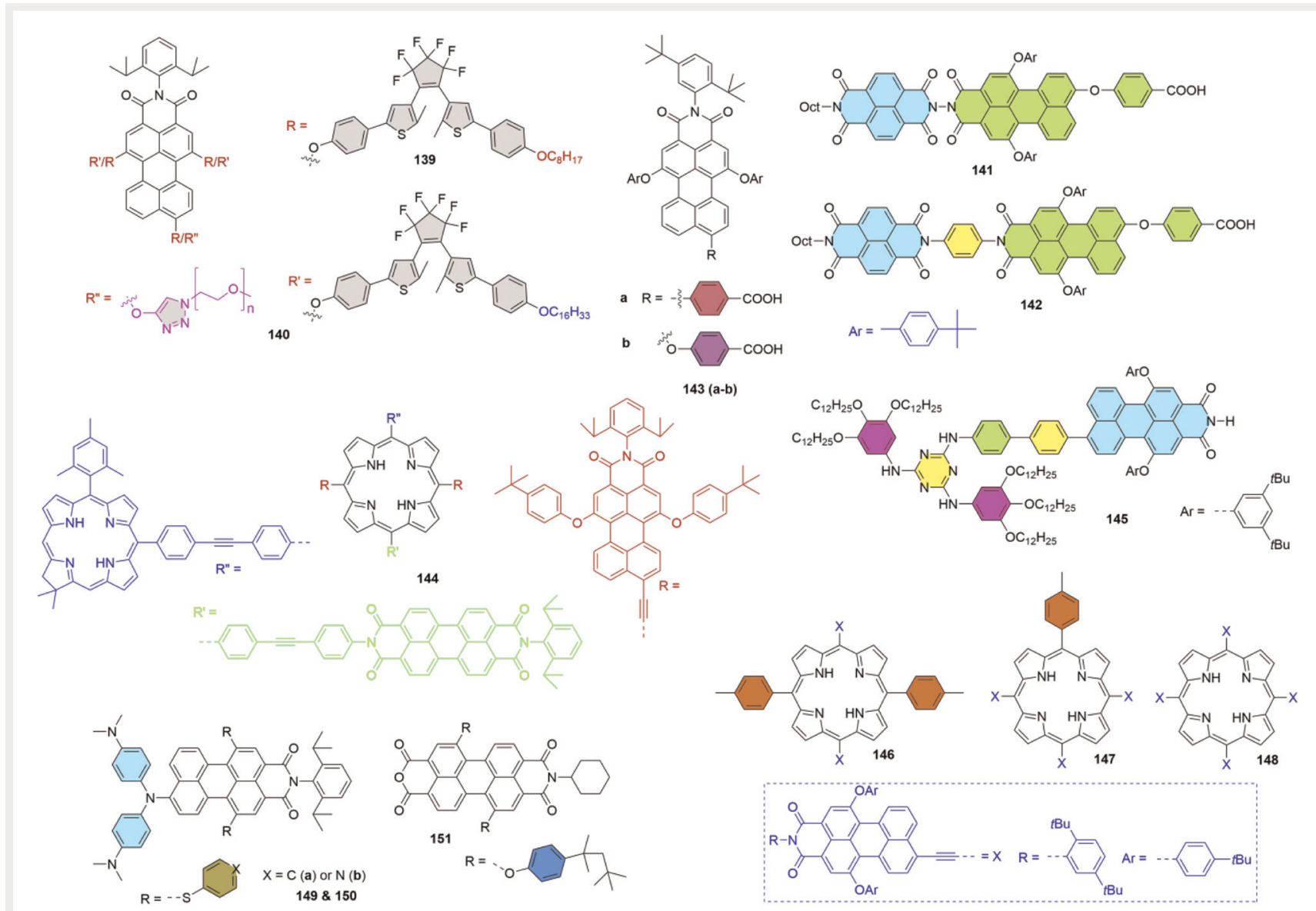

Figure 20 Examples of both bay- and peri-functionalized PMls.

(C9-position) of PMI was substituted with the electron-donating group and the bay region bears the 4-tert-butylphenoxy group (entry 7), the absorption maximum shows a redshift of $28 \mathrm{~nm}$ in comparison to PMI. A large shift in the absorption maximum is also observed when an electron-donating amine group was introduced at 9-position. Compounds 54 and 55 (entries 13 and 14) show a large red-shift due to the substitution with the electron-donating group. Due to the conjugation of electron pair on the donor atom with the PMI core, the chromophore behaves as an intramolecular charge transfer dye. With the introduction of an electron-donating substituent at the peri-position (C-10 of PMI, see entry 16 for compound 126), the absorption maximum shifted further to a longer wavelength region.

Another important factor, which can significantly modify optical properties of flat $\pi$-conjugated PMIs and PDIs is the formation of extended aggregated structures with specific topologies in solution. Aggregates are classified as $\mathrm{H}$ - and Jdepending on the dipole orientation. In J-aggregates, neighbouring chromophores are oriented in a head-to-tail fash- ion, resulting in a negative excitonic coupling, while in $\mathrm{H}-\mathrm{ag}-$ gregates nearest-neighbour molecules are oriented in a side-by-side manner, resulting in a positive excitonic coupling. The energetic ordering of the excitons has profound effects on the photophysical response and this has been reviewed recently. ${ }^{14 a, 15,45}$

\section{Singlet Fission Properties}

SF is recognized as an appealing photophysical approach to harvest triplet state of an organic chromophore that leads to boost in the PCE value of OSCs up to $46 \%$ beyond the Shockley-Queisser limit (33\%). We can find a lot of examples for PDI-based SF materials in the literatures, whereas PMI has barely been used hitherto in SF. To become an ideal SF material, the desired chromophore must satisfy the energy requirement of low-excited singlet and triplet states i.e., $E$ $\left(\mathrm{S}_{1}\right)=2 E\left(\mathrm{~T}_{1}\right)$. The theoretically calculated singlet and triplet energies of mostly used parent PDIs are $2.36 \mathrm{eV}$ and 1.10 
Table 1 Absorption and emission properties of PDI and PMI derivatives to understand the effect of substitution

\begin{tabular}{|c|c|c|c|c|c|c|}
\hline Entry & Rylene dye & Solvent & $\lambda_{\mathrm{Abs}}^{\mathrm{Max}} / \mathrm{nm}$ & $\lambda_{\mathrm{Em}}^{\mathrm{Max}} / \mathrm{nm}$ & Stokes-shift/nm & Quantum yield \\
\hline 1 & 1 & Cyclohexane & 435 & 435 & 0 & 0.94 \\
\hline 2 & Perylene mono-anhydride & $\mathrm{CHCl}_{3}$ & 487 & 544 & 57 & - \\
\hline 3 & 2 & Toluene & 504 & 526 & 22 & 0.48 \\
\hline 4 & 9 & Toluene & 507 & 529 & 22 & 0.91 \\
\hline 5 & N-Benzyl-PDI & $\mathrm{CHCl}_{3}$ & 527 & 538 & 11 & 0.98 \\
\hline 6 & N-Bu-PDI & Toluene & 528 & 537 & 9 & 0.97 \\
\hline 7 & 9-(4-Tert-butylphenoxy)-PMI 9 & Toluene & 532 & 567 & 35 & 0.82 \\
\hline 8 & 1,6,9-Tris(4-tert-butylphenoxy)-PMI 9 & Toluene & 536 & 577 & 41 & 0.86 \\
\hline 9 & 14 & Toluene & 523 & 560 & 27 & 0.77 \\
\hline 10 & 16 & Toluene & 515 & 545 & 30 & 0.99 \\
\hline 11 & 17 & Toluene & 520 & 565 & 45 & 0.98 \\
\hline 12 & 18 & Toluene & 535 & 627 & 92 & 0.71 \\
\hline 13 & 54 & Toluene & 568 & 665 & 97 & 0.02 \\
\hline 14 & 55 & Toluene & 616 & 697 & 81 & 0.34 \\
\hline 15 & 64 & Toluene & 521 & 551 & 30 & 0.99 \\
\hline 16 & 126 & Water & 567 & 742 & 175 & 0.17 \\
\hline
\end{tabular}

$1.28 \mathrm{eV}$ respectively, which have made them preferable candidate over PMI. But it can be foretold, the required singlet and triplet energy value can be achieved by structural modification of PMI derivatives, more importantly on peri-functionalization of PMI. Inspired by this, Guldi and co-workers have successfully utilized PMI for an ultrafast singlet-fission process. ${ }^{46}$ They designed and prepared a naphthalenelinked PMI-ester dimer (152) containing long alkyl chains from the understanding of singlet $(2.39 \mathrm{eV})$ and triplet $(1.12 \mathrm{eV})$ energy of PMI (see Figure 21). ${ }^{46}$ In this contribution, the authors show that PMI-ester dimer exhibits a spin-allowed SF process, in which transformation of singlet

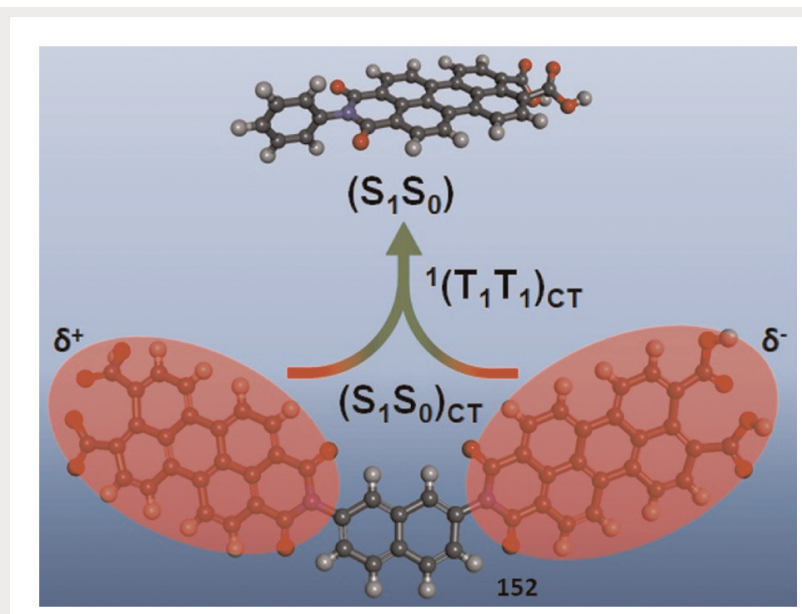

Figure 21 Representation of SF by naphthalene-coupled PMI-ester dimer. Reprinted with permission from Ref. 46. Copyright 2020 American Chemical Society.
$\left(\mathrm{S}_{1} \mathrm{~S}_{0}\right)$ to triplet ${ }^{1}\left(\mathrm{~T}_{1} \mathrm{~T}_{1}\right)$ pairs occurred via formation of an intermediate charge transfer state that has both excited-state charge-transfer characteristics, represented as $\left(\mathrm{S}_{1} \mathrm{~S}_{0}\right)_{\mathrm{CT}}$ and ${ }^{1}$ $\left(\mathrm{T}_{1} \mathrm{~T}_{1}\right)_{\mathrm{CT}}$, corroborated by femtosecond transient spectroscopy. The triplet-triplet decorrelation and stability of 1 $\left(\mathrm{T}_{1} \mathrm{~T}_{1}\right)_{\mathrm{CT}}$ were perturbed at room temperature (RT) which in turn formed the upconverted singlet state $\left(\mathrm{S}_{1} \mathrm{~S}_{0}\right)_{\mathrm{Uc}}$. Thus, they found a competition between the down-conversion SF and up-conversion geminate recombination of triplet pairs at RT. At lower temperature (140 K), SF-mediated triplet pair formation was more favourable than that at RT (298 K), and QY (triplet) values were $4.2 \%$ at RT and $14.9 \%$ at $140 \mathrm{~K}$, respectively. Theoretical calculation also evinced that the SF process was thermodynamically favourable by a negative free energy value of $0.32 \mathrm{eV}$.

\section{Förster Resonance Energy Transfer with PMI}

Förster resonance energy transfer (FRET), often termed as 'spectroscopic ruler' is described as energy transfer between donor and acceptor moieties of a single chromophore, whose efficiency depends on spectral properties of the donor and acceptor, their mutual distance and orientation. PMI dye is a lucrative choice for constructing a FRET pair as unveiled by Zenobi et al. in their contribution in 2019.47 They investigated the FRET mechanism by synthesizing kinked (108a) and linear (108b) dyads constituting of two PMI units and oligo(phenyleneethynylene)s as a spacer between them. Native PMI and tri-aryloxy PMI derivative were considered as donor and acceptor respectively in the energy transfer process based on the maximum overlap of their 


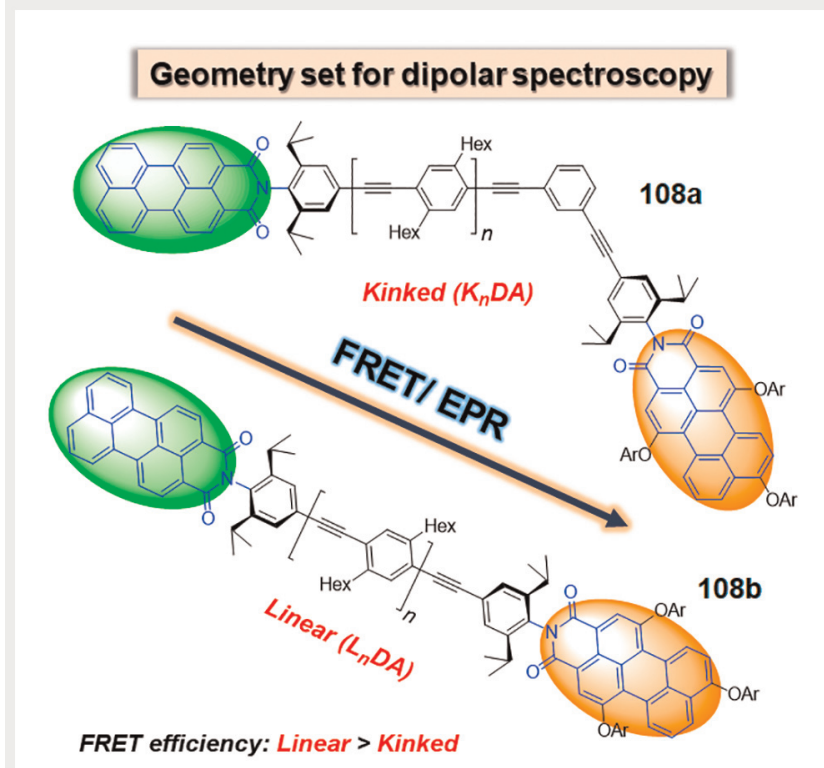

Figure 22 Illustration of FRET in PMIs-oligo(phenyleneethynylene) system. Reprinted with permission from Ref. 47. Copyright 2019 American Chemical Society.

emission and absorption spectra. This work has documented that with increasing the number of spacer units, donor emission enhanced which denoted lowering of energy transfer with increasing distance. The linear derivative showed a higher FRET efficiency compared to its kinked isomer (see Figure 22). ${ }^{47}$

\section{Symmetry Breaking Charge Transfer Properties}

To shed light on PMI-based symmetry breaking charge transfer (SBCT), Hariharan's group came up with a PMI dimer model where two PMI monomers were directly connected with an orthogonal orientation between them. ${ }^{48}$ The PMI dimer (133) exhibited fluorescence quenching $\left(\varphi_{\mathrm{F}}=0.21\right)$ in polar solvent acetonitrile (ACN) which is attributable to photoinduced SBCT, as depicted in Figure 23. Femtosecond transient study unveiled that the occurrence of fast SBCT $\left(\tau_{\mathrm{cs}}=2.8 \mathrm{ps}\right)$ in ACN is facilitated by the formation of a charge-separated radical ion-pair state in the excited state.

Contrarily, in non-polar solvents such as cyclohexane, a PP dimer became highly emissive $\left(\varphi_{\mathrm{F}}=0.68\right)$ due to conformational planarization-induced population of $S_{1}$ excitation state. The time-constant of conformational planarization in cyclohexane was very short $\left(\tau_{\mathrm{cp}}=20 \mathrm{ps}\right)$. Conformational planarization aided in adopting a more planar geometry in

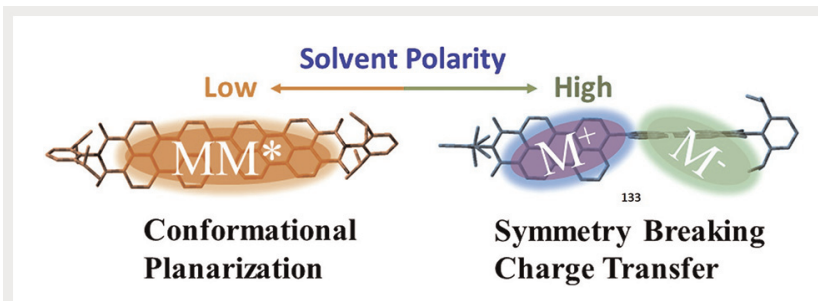

Figure 23 Demonstration of SBCT and fluorescence property from head-to-head coupled PMI dimer. Reprinted with permission from Ref. 48. Copyright 2020 American Chemical Society.

the excited state where electronic coupling between two monomers becomes very feasible, leading to slower SBCT. This contribution exemplified that the highly twisted PP dimer showing significant SBCT may act as a promising material to achieve high PCE in OSC devices.

\section{Panchromatic Light Absorption Properties of PMI}

It is well researched that panchromatic light absorption is regarded as a prime factor for effective charge separation, thus accelerating solar cell efficiency. Hence, design and synthesis of highly conjugated multichromophoric architectures become an area of paramount interest to the scientists. Inspiringly, Lindsey's group developed a star-shaped pentad (144) comprised of porphyrin and PMI as a light absorber, PDI as an electron acceptor and chlorin as a hole trapping agent connected via ethyne linkage, as illustrated in Figure $24 .{ }^{49}$ The triad made of one porphyrin and two PMIs via ethynyl spacer exhibited bathochromically shifted emission similar to tetra-aryl-porphyrin with an enhanced fluorescence QY in solution. This panchromatic array was prepared by multistep synthetic pathways where the building units were amalgamated together via Pd-catalyst-mediated Sonogashira cross-coupling. Upon excitation of the entire scaffold, the central triad (PMI-Por-PMI) absorbs the sunlight in the UV-Vis region (below $400 \mathrm{~nm}$ to above $700 \mathrm{~nm}$ ), which is followed by a facile excited state energy transfer to the lowest energy state, $S_{1}$ state of tetrapyrrole-like array. It is to be noted here the overall charge separation process led to yield a radical-ion pair state (chlorin as radical cation and PDI as radical anion) in the excited state. Here, PMIs played a vital role in panchromatic light absorption. Working along the similar lines, the same group reported PMI and porphyrin-constituted multichromophoric arrays (146, 147, and 148) that serve as an alternative model of panchromatic light absorption. The structures are provided in the Figure 25.50 


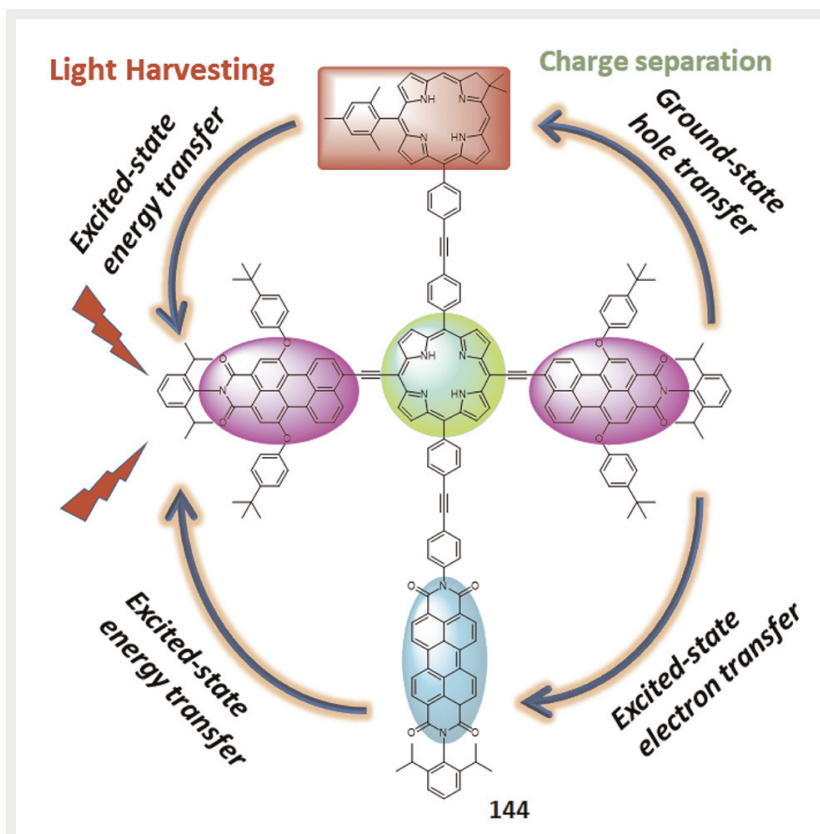

Figure 24 Representation of molecular structure of porphyrin-PMI pentad and several photophysical processes.

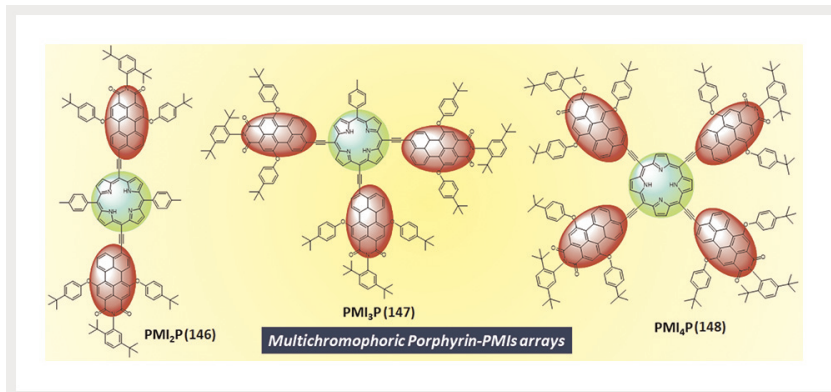

Figure 25 Structural illustration of PMI-porphyrin-based multichromophoric systems.

\section{Acid/Base Sensitivity of PMI}

The photo-physics of PMI-embedded donor-acceptor derivatives is highly sensitive to solvent polarity as well as acid/ base, established by the contribution from Brouwer's group. ${ }^{51}$ Two D-A compounds, i.e. PMI-dHQD (122a) and PMI-BnCPD (122b), were synthesized by coupling donor 9benzylcupreidine (BnCPD) and dehydroquinidine (dHQD) with a PMI acceptor via 9-position of PMI chromophore, as shown in Figure 26. ${ }^{51}$

The fluorescence QY and excited-state lifetime of both derivatives substantially decreased with increasing solvent polarity scale; $\varphi_{\mathrm{F}} \sim 0.77$ (toluene) to $\sim 0.03$ (DMF) and $\tau_{\text {avg }}$ $\sim 4.0 \mathrm{~ns}$ (toluene) to $\sim 0.2 \mathrm{~ns}$ (DMF). Both compounds dis-

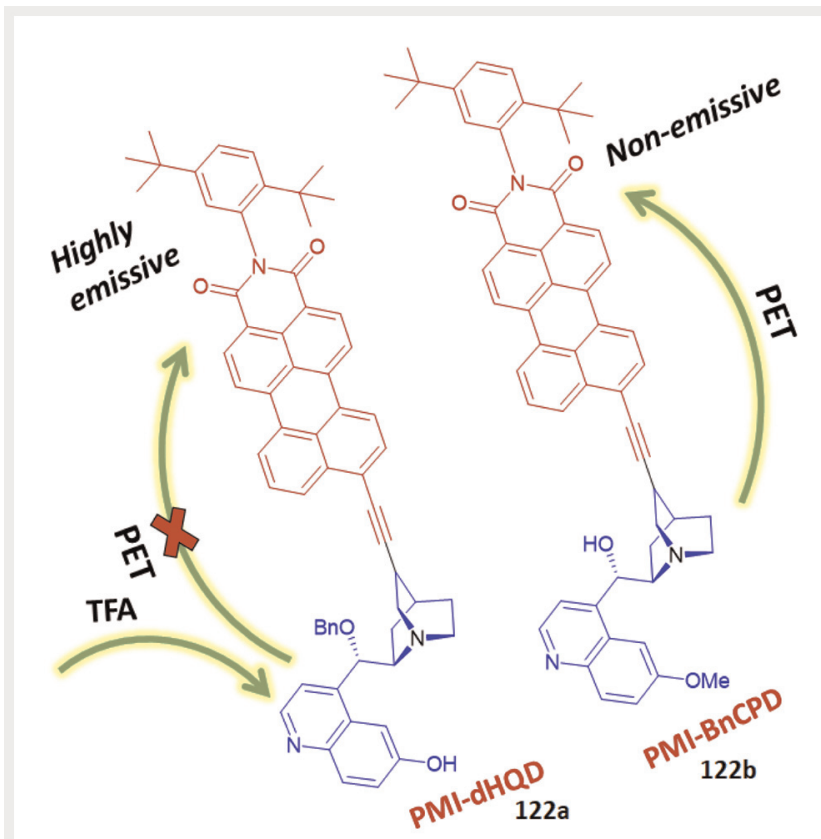

Figure 26 Demonstration of PET process in PMI-alkaloid derivatives and effect of acid/base in this photophysical process.

played weak fluorescence character in polar solvents because of charge separation (timescale $0.21 \mathrm{~ns}$ ) between donor and acceptor moieties in excited state and stabilization of charge separated state in polar solvents. On the other hand, upon addition of trifluoro acetic acid (TFA), the highly emissive property of both compounds reinstated in polar solvents as protonation of $\mathrm{N}$-atom in donor moieties prevented donor to acceptor electron transfer that is responsible for charge separation.

\section{NIR-Absorbing PMI}

Achieving absorption in the NIR region (700-2000 nm) by organic chromophores appears to be a challenging task, therefore it has aroused interest among the scientific clan to synthetically develop new NIR-absorbing dyes. Installing donor-acceptor moieties in a single organic skeleton to increase $\pi$-conjugation dramatically is a customary approach to accomplish both NIR absorption and emission in solution. Fusion of an aromatic ring in a $\pi$-conjugated dyad is another pathway to make a NIR-absorbing material, established by Wu et al. They reported two doubly and triply fused porphyrin-PMI hybrid dyads 128 and 129, as given in Figure 27a, prepared by Suzuki coupling and followed by cyclization by $\mathrm{FeCl}_{3}$ in DCM. ${ }^{52}$ Pleasingly, both NIR-absorbing compounds were extremely photostable having larger dipole moment character. Figure 27b displays that compound 128 

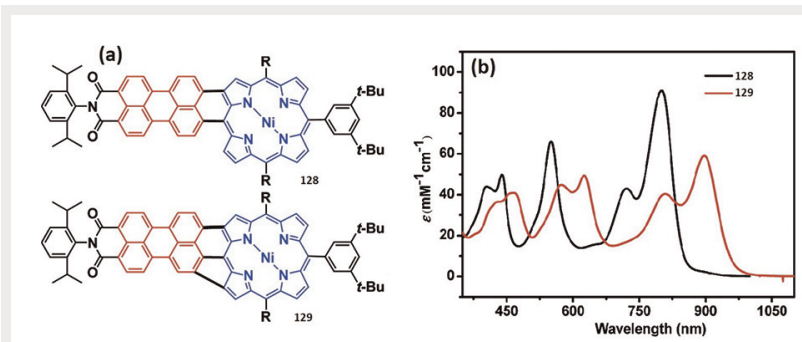

Figure 27 (a) Structural representation of PMI-porphyrin fused derivatives. (b) UV-Vis spectra of two derivatives in solution. Reprinted with permission from Ref. 52. Copyright 2011 American Chemical Society.

exhibited an absorption maximum at $803 \mathrm{~nm}$ whereas the $\lambda_{\text {max }}$ of triply-linked 129, which is an extended fused analogue of 128, was tremendously red-shifted (94 nm), compared to 128 presumably via augmentation of $\pi$-conjugation in the fused architecture. This work conveys that such perifused PMI in conjunction with porphyrin dye may serve as a potential optoelectronic material in future organic photovoltaics.

Almost 20 years back, Müllen and co-workers introduced a 1,5-diaminoanthraquinone-appended PMI-based NIR-absorbing chromophore which was remarkably thermally stable as well as photostable. ${ }^{53}$ The UV-Vis profile of this derivative demonstrated presence of two absorption bands, positioned at $618 \mathrm{~nm}$ in the visible region and another one at $1000 \mathrm{~nm}$ in the NIR region. The NIR-band at $1000 \mathrm{~nm}$ is broad and structureless arose due to intramolecular charge transfer effect, a stark contrast to the well-defined sharp $618 \mathrm{~nm}$ band. The formation of intramolecular H-bond between $\mathrm{N}-\mathrm{H}$ and $\mathrm{C}=\mathrm{O}$ of anthraquinone led to elimination of the solvatochromic property of this compound. Finally, the authors postulated the real application of this PMI-based NIR-absorbing chromophore in thermal imaging and optical data storage.

Instead of making larger donor-acceptor conjugated dyads/triads mentioned above, procuring of NIR-absorption from a small conjugated scaffold is always desired as it is devoid of troublesome synthesis. Needless to say, a PMI dye provides such generous opportunities to meet this demand. Recently, thermally stable N-doped aza-PMI derivatives abbreviated as APMI-OH (79), APMI-N (80) and APMI-K (81), as represented in Figure 28a, have been synthesized which displayed the maximum absorption in the 700-900 nm region. ${ }^{38}$ This intense NIR-absorption can be perceived as an outcome of the strong 'push-pull effect' from the donor phenolate to the electron-deficient imide position. Figure 28b demonstrates that both ionic derivatives APMI-N and APMI-K exhibited extraordinary red-shifted absorption centred on $796 \mathrm{~nm}$ and $787 \mathrm{~nm}$ respectively compared to APMI-OH that denotes the push-pull effect becoming stronger in ionic analogues. They also synthesized PMI-OH and

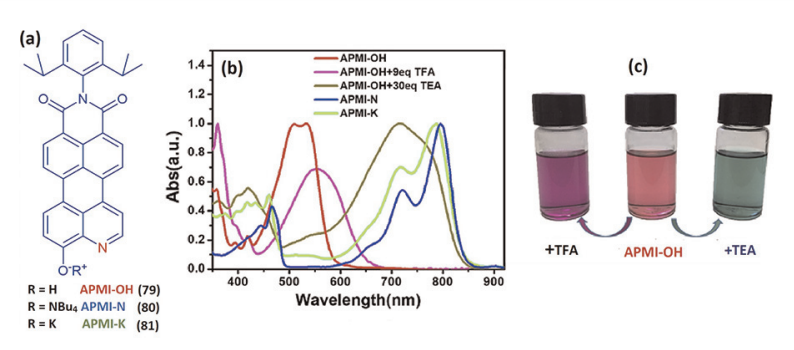

Figure 28 (a) Chemical structure of N-doped PMI derivatives, (b) absorption spectra of three derivatives along with change of spectra of APMI-OH in presence of TFA and TEA, and (c) digitally captured vial images of APMI-OH, APMI-OH + TFA, APMI-OH + TEA solutions. Reprinted with permission from Ref. 38. Copyright 2020 Elsevier.

observed that PMI-OH mostly absorbs nearby the $500 \mathrm{~nm}$ region and not in the NIR region, thus clarifying a crucial role of $\mathrm{N}$ atom in tuning the absorption property. Addition of TFA/TEA in APMI-OH solution tailored their optical properties and changed the color of solution, as seen in Figure 28 (b, c), implying the great sensitivity of these compounds in acid/base environment. Further, density functional theory calculations unveiled that protonation of carbonyl $\mathrm{O}$ and $\mathrm{O}^{-}$ is mainly responsible for this NIR property. ${ }^{38}$

\section{Achieving Triplet State Using PMI}

Accessing a triplet state using a suitable organic chromophore is a crucial phenomenon in organic electronics specifically in organic light-emitting devices. An unsubstituted PMI chromophore is inadequate for achieving of lower energy triplet state, so, the prevailing approach to activate triplet-state characteristics of such derivatives will be to conjoin a native PMI with triplet sensitizers or heavy metals to foster intersystem crossing (ISC). By following this concept, Castellano's group introduced a metal character in a PMI derivative by covalent conjugation between PMI and platinum terpyridine complex via an acetylene linkage, abbreviated as PMI-Pt-trpy (121) given in Figure 29a. ${ }^{54}$ They observed quenching of emission from the singlet excited state of PMI and also, it was observed that the presence of triplet oxygen $\left({ }^{3} \mathrm{O}_{2}\right)$ significantly reduced the fluorescence emanating from the whole complex. Although they did not procure any phosphorescence of the PMI-Pt-trpy derivative, they were successful in measuring the photoluminescence spectra of ${ }^{1} \mathrm{O}_{2}$ showing emission at $1270 \mathrm{~nm}$ as depicted in Figure 29b, which denotes that the metal-ligand complexappended PMI compound had acquired a long-lived triplet state with a lifetime of $8.4 \mu \mathrm{s} .^{54}$

In 2018, Li and co-workers reported another pathway of harvesting of triplet states rather than the customary approach of incorporating a heavy metal in a chromophore. ${ }^{55}$ 


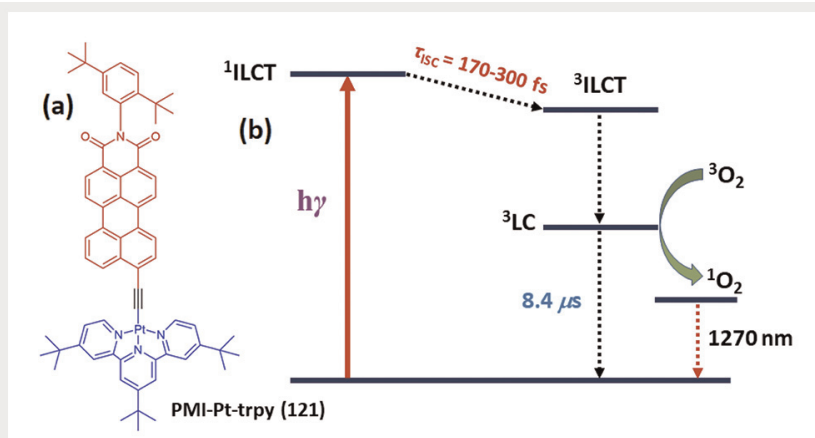

Figure 29 (a) Structure of PMI-Pt-trpy and (b) diagram illustrating the photophysical processes occurring to harvest the triplet state.

They synthesized two novel phenothiazine-appended PMI derivatives conjugated via $\mathrm{C}-\mathrm{N}$ and $\mathrm{C}-\mathrm{C}$ bond formation abbreviated as PMI-N-PTZ (117a) and PMI-C-PTZ (117b). In between both compounds, PMI-N-PTZ possessed an orthogonal geometry (dihedral angle $95^{\circ}$ ), ideal for ISC process. It was depicted that PMI-N-PTZ exhibited a better efficiency in spin-orbit charge separation-mediated ISC (SOCS-ISC) with higher triplet-state production (57\%) over its C-linkage derivative. They demonstrated that the overall molecular geometry, vector dipole orientation of two chromophores and solvent polarity played immense roles to achieve a long-lived stable triplet state (lifetime $200 \mu \mathrm{s}$ ), as represented in Figure 30. Interestingly, they observed that no effective SOCS-ISC occurred in the bay-substituted PDI-PTZ derivative which established PMI-N-PTZ as a more efficient triplet harvester than its PDI analogues.

Spin-orbit charge transfer-mediated ISC (SOCT-ISC)-mediated accessing of triplet states for donor-acceptor dyads is majorly dependent on the electron-donating capability of the donor moieties in concord with the mutual orientation and distance between the donor and acceptor moieties, which encouraged the same group to further explore in this direction. Furthermore, they investigated the SOCT-ISC property on four newly developed PMI-carbazole dyads abbreviated as PMI-Cz (153), PMI-Cz-1 (154), PMI-Cz-2 (155) and PMI-Cz-tBu (137), as given in Figure 31a. Figure 31b displays the solvent-polarity-dependent photophysical studies which disclose that the emission spectra of these dyads were intensified in non-polar solvent hexane, but they exhibited dramatic quenching of emission with the appearance of red-shifted structureless fluorescence spectra in polar solvent ACN due to photo-induced electron transfer (PET). ${ }^{56}$

The charge separation and charge recombination took place at the timescale of $0.28 \mathrm{ps}$ and $1.21 \mathrm{~ns}$ respectively as estimated from femtosecond transient absorption study (Figure 31c). Moreover, they achieved the lower energy triplet state with a lifetime of $14.7 \mu$ s and QY of $2.7-16.3 \%$,

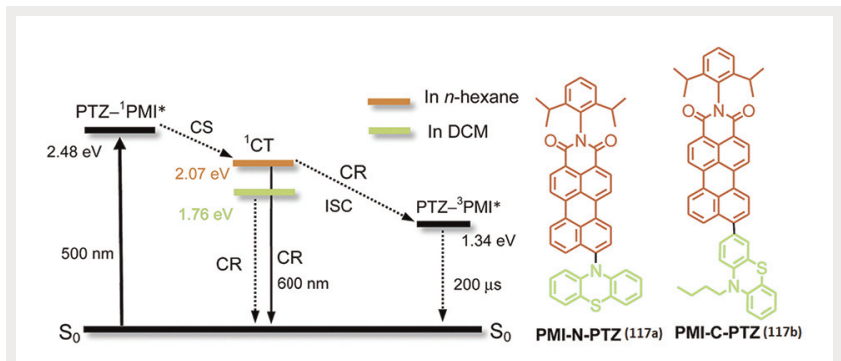

Figure 30 Schematic representation of excited-state photophysics for accessing triplet states by PMI-N-PTZ and PMI-C-PTZ. Reprinted with permission from Ref. 55. Copyright 2019 Royal Society of Chemistry.

wherein they found that formation of this triplet is immensely influenced by solvent polarity, which is a symbolic feature of SOCT-ISC. Here, the SOCT-ISC rates of PMI-carbazole candidates were comparatively less than that of previously obtained PMI-N-PTZ, which may be ascribed to the relatively less donor strength of carbazole and reduction in the constrained geometry.

Recently, Yin and co-workers have developed a novel carboxylesterase (CE)-responsive assembly (FHP) using tetrachloroperylene monoimide (109) and folate-decorated albumins ${ }^{57} \mathrm{FHP}$ nanoclusters have a size of $\mathrm{ca} .100 \mathrm{~nm}$ diameter. Tumor-specific CE hydrolyzes $\mathbf{1 0 9}$ and disassembles FHP into ultrasmall nanoparticles (NPs; $c a .10 \mathrm{~nm}$ ) and thereby facilitates the deep tumor penetration of FHP. Moreover, such CE-triggered assembly leads to 8-fold enhancement in the fluorescence intensity for NIR fluorescence (NIRF) imaging and 4-fold elevation of singlet oxygen generation for photodynamic therapy. The CE-responsive 109 was decorated suitably using hydrophilic galactose for tumor targeting as well as with the substrate of $\beta$-alanine methyl ester. With this molecular design the authors have demonstrated
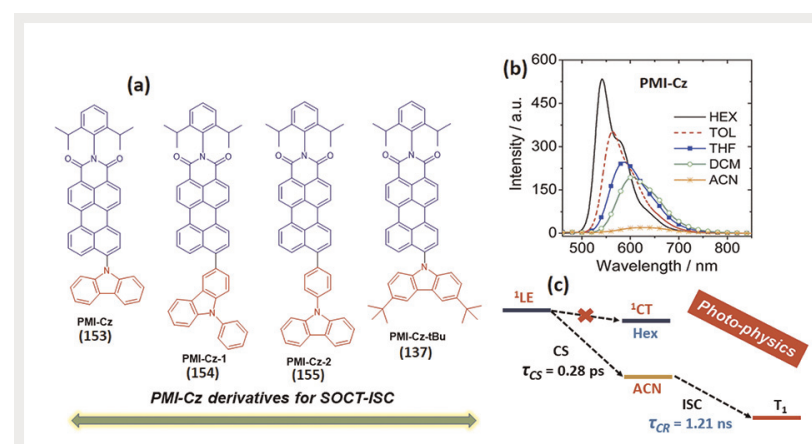

Figure 31 (a) Structural representation of PMI-carbazole derivatives, (b) emission spectra of PMI-Cz (153) in different solvents, (c) schematic diagram for representation of the SOCT-ISC process. Reprinted with permission from Ref. 56. Copyright 2020 Royal Society of Chemistry. 


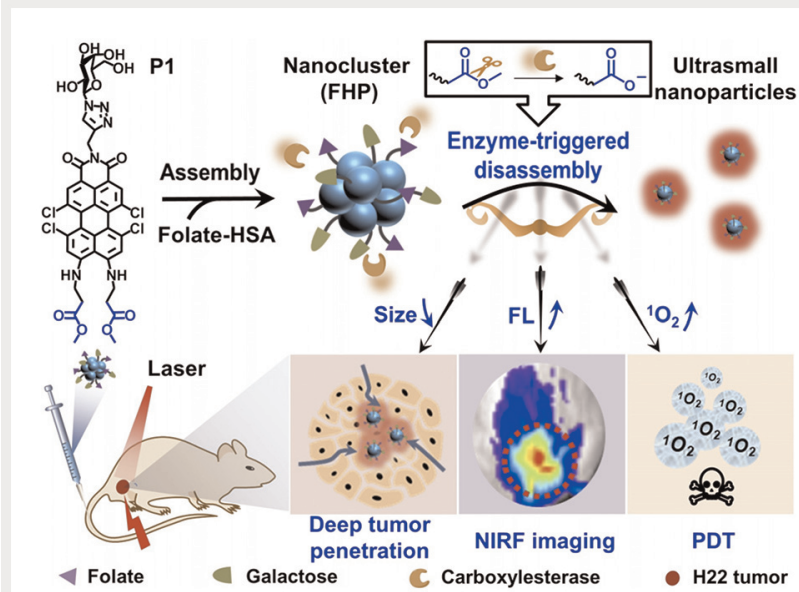

Figure 32 Schematic demonstration of carboxyesterase-responsive nanocluster fabrication using assembly of 109 and folate-modified human serum albumin (folate-HSA) for deep tumor targeting, and NIRFbased imaging-guided photodynamic therapy. Reprinted with permission from Ref. 57. Copyright 2019 Wiley-VCH.

a stimulus-responsive NIRF with tumor targeting ability and photodynamic action as shown in Figure 32.

\section{Solid-State Emissive PMI}

Highly planar and $\pi$-conjugated PMIs are strongly emissive in solution but they hardly fluoresce in the solid state or the aggregated state. The examples of PMI-based solid-state emissive compounds are scarcely reported in the literature. Lately, Fang and co-workers added a novel peri-coupled PMI-o-carborane derivative in the gallery of PMI-based solid-state emissive compounds. This PMI-o-carborane (156) derivative displayed strong bright red emission with appreciable QY (40.7\%) in the solid state with respect to a reference PMI-acetylene compound, as shown in Figure 33a. ${ }^{58}$ The appearance of strong emission in the powder form is probably due to the inhibition of $\pi-\pi$ stacking interaction in the solid state which usually promotes the formation of non-fluorescent $\mathrm{H}$-aggregates. Additionally, they observed a systematic red-shift in the emission spectra on transition from solution (acetone) to the crystal state via thin-film and powder forms, $\lambda_{\max }$ (acetone) $-560 \mathrm{~nm}$ and $\lambda_{\max }$ (crystal) $-670 \mathrm{~nm}$, the combined spectra are provided in Figure 33b. The authors have speculated that J-aggregation-mediated ICT between neighbouring PMI-o-carborane derivatives is predominantly responsible for such red-shift in emission spectra. Crystal structure revealed that PMI-o-carborane compounds created a discrete dimer where the overlap between the two monomers is not that effective (overlap efficiency $58 \%$ ) possibly provoking them to fluoresce in the solid state.
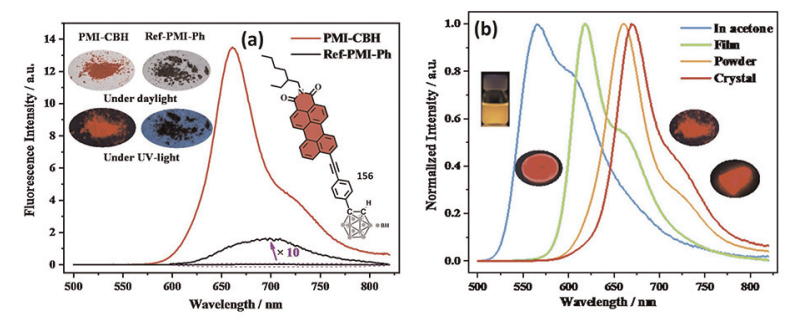

Figure 33 (a) Fluorescence spectra of PMI-CBH and Ref-PMI-Ph in the powder form; the inset shows the digital images of both compounds under daylight and UV-light. (c) Normalized emission spectra of PMI-CBH in different states. Reprinted with permission from Ref. 58. Copyright 2020 Elsevier.

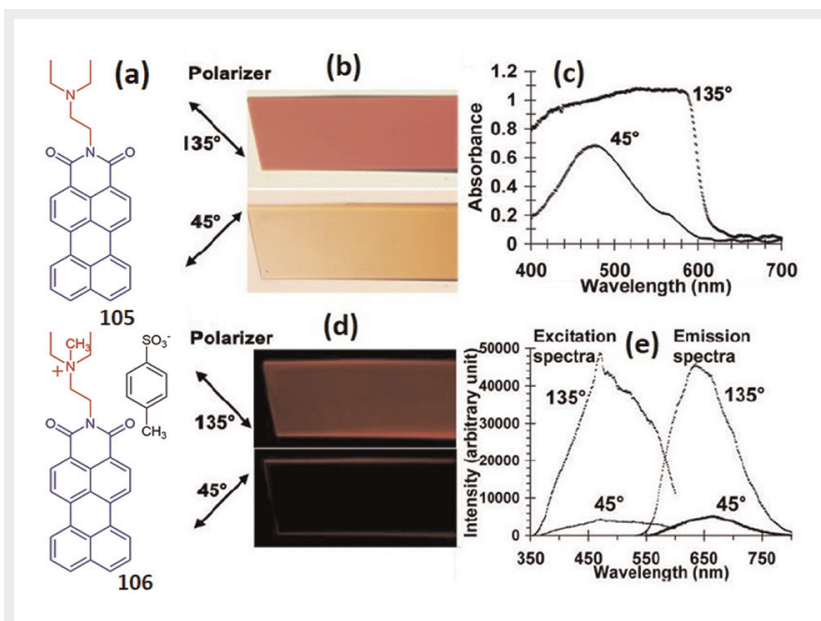

Figure 34 (a) Chemical structures of PMI derivatives used in this investigation, (b) Optical micrographs showing the anisotropic absorption of light by a crystal of 106 viewed under an optical microscope. (c) Spectra showing the absorption of polarized visible light by the crystal of 106. (d) Optical micrographs of the anisotropic fluorescence emission from the crystal; the inset shows the structure of 106, (e) Excitation spectra (acquired at $\lambda_{\mathrm{em}}=632 \mathrm{~nm}$ ) and the emission spectra (acquired at $\lambda_{\text {ex }}=467 \mathrm{~nm}$ ) of the crystal. Reprinted with permission from Ref. 59. Copyright 2007 Wiley-VCH.

A few years ago, Chang's group discovered dichroic absorption and anisotropic emission properties of tape-like PMI crystals for which they synthesized an ionic PMI derivative (106) by quaternization of a tertiary amine of incorporated amine chain in the imide position of compound $\mathbf{1 0 5}$, the structures are given in Figure 34a..$^{59}$ The direction of electronic transition was aligned at $135^{\circ}$ and $45^{\circ}$ angles with respect to the principal axis of the crystal (Figure 34b). In the UV-Vis spectra of the crystal (Figure 34c), strong absorption with spectral broadening was observed when the polarization axis of light was fixed at $135^{\circ}$, which made a monumental difference to the instance when it was kept at $45^{\circ}$. In- 


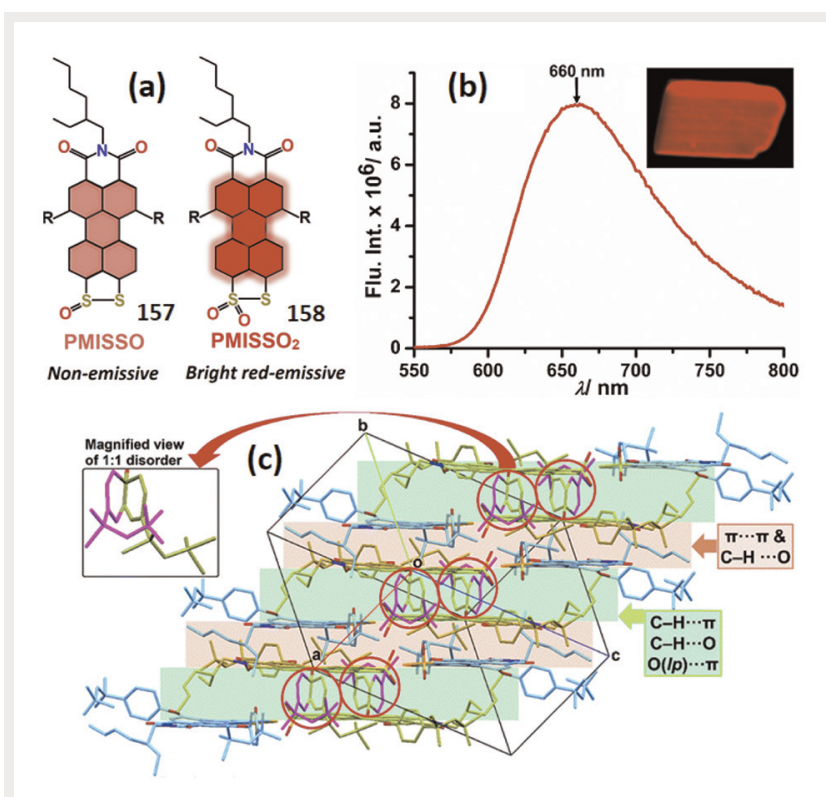

Figure 35 (a) Demonstration of chemical structures of two PMI derivatives along with their emissive behaviour in the solid state; (b) emission spectra of $\mathrm{PMISSO}_{2}$ in the powder state; inset depicts the microscopic images of $\mathrm{PMISSO}_{2}$ crystal; (c) solid-state crystal packing arrangement of $\mathrm{PMISSO}_{2}$ and representation of various intermolecular interactions. Reprinted with permission from Ref. 60. Copyright 2016 Royal Society of Chemistry.

evitably, an intense emission (573-648 nm) of the crystal was obtained upon excitation at the $530-560 \mathrm{~nm}$ region when the emission polarizer was oriented along the same axis of maximum absorption (Figure 34d,e). The intense red emission from the ionic crystal can be thought of as an upshot of intermolecular orbital interaction and excitonic coupling among neighbouring PMI molecules. Crystal packing unveiled an antiparallel arrangement of ionic monomers spawned to reduce the repulsion between quaternized ternary ammonium groups. The electrostatic interaction between cationic ammonium and surrounding counterion anionic sulfonate was the driving force to stabilize the crystal.

Synthetic chemists have mastered the tedious synthetic pathways to make solid-state fluorescent PMI/PDI derivatives. To avoid such tedious syntheses, lately, our group has brought an innovative idea to obtain a solid-state-emissive PMI derivative, i.e. fluorometric and ratiometric detection of organic peroxides mediated origin of bright red emissive new PMI compound. Actually, the authors acquired two solid-state-annulated oxidized derivatives of PMI abbreviated as PMISSO (157) and $\mathbf{P M I S S O}_{2}$ (158) upon oxidation of PMISS organic peroxide (Figure 35a) ${ }^{60}$ Amongst the two derivatives, $\mathbf{P M I S S O}_{2}$ showed intense fluorescence in solution as well as in solid state, contrarily PMISSO was reluctant to exhibit any such emission in both states. The absorption spectra of $\mathbf{P M I S S O}_{2}$ powder exhibited appearance of a number of vibronic progressions covering the $300-700 \mathrm{~nm}$ region. Consequently, upon exciting at $517 \mathrm{~nm}$, the same compound displayed an intense broad emission spectrum centred on $660 \mathrm{~nm}$ as seen in Figure 35b, with QY 0.14 in the solid state. The origin of such emission feature in the solid state was expounded from the crystallographic studies which delineated that back-and-forth molecular movement of the bulky tert-octyl phenoxy substituent at the bay-position caused greater twisting of the PMI core. It is also evident from Figure $35 \mathrm{c}$ that the strong $\pi-\pi$ interaction of the PMI core was minimized by the replacement of $\mathrm{C}-\mathrm{H} . . . \pi$, $\mathrm{C}-\mathrm{H} . . . \mathrm{O}$ between $\mathrm{SO}_{2}$ and bulky bay-substituents and $\mathrm{lp}(\mathrm{O})$ $\ldots \pi$ interactions. This contribution also conveys the solidstate structure-property relationship in the arena of PMI relevant to organic optoelectronic applications.

\section{Thermo-Responsive Materials with PMI}

The above discussions illuminate the unique photophysical properties of PMI in solution as well as in the solid state. Rather than the photophysical properties, PMI has also captured significant attraction on its thermo-responsive behavior as disclosed by Wang's group in 2019 (Figure 36a). ${ }^{61}$ To probe the same, they purposefully made a peri-linked PMI functionalized with permethyl- $\beta$-cyclodextrin named BPMI-CD, here PMI and CD play their roles as a fluorophore and a water-solubilizing agent, respectively.

Initially, the aqueous solution of BPMI-CD was clear at RT, and interestingly with increasing temperature up to $60^{\circ} \mathrm{C}$, the clear solution turned into turbid with showing a lower critical solution temperature (LCST) value at $42.0^{\circ} \mathrm{C}$ (Figure $36 \mathrm{~b})$. Again, upon cooling down the solution, LCST was obtained as $34.4^{\circ} \mathrm{C}$, determined from UV-Vis spectroscopy. In addition to that, they performed concentration-dependent thermo-responsive behavior analysis, which manifested the LCST values lowered with increasing concentration (Figure 36c). They hypothesized that the thermo-responsive behavior may be mainly ascribed to the self-inclusion effect of cyclodextrin units. Excitingly, upon addition of a competitive guest, TPPS, the turbid solution became clear because of strong TPPS-CD complex formation mediated release of bay-phenyls from the self-inclusion complex. Further, the introduction of the PMCD-Ts host snatched its TPPS guest to form the PMCD-TS-TPPS complex which helps to regain the turbidity in solution.

Wang and co-workers have developed a biperylene monoimide (BPMI) derivative with a conjugated lactosebased glycodendrimer (BPMI-18Lac, see Figure 37) for demonstrating the cooling-induced emission (CIE) enhancement. ${ }^{62}$ BPMI-18Lac exhibited the CIE phenomenon with a 7-fold intensity enhancement when the temperature changed from 80 to $-40^{\circ} \mathrm{C}$ (Figure 37, top part). Such en- 

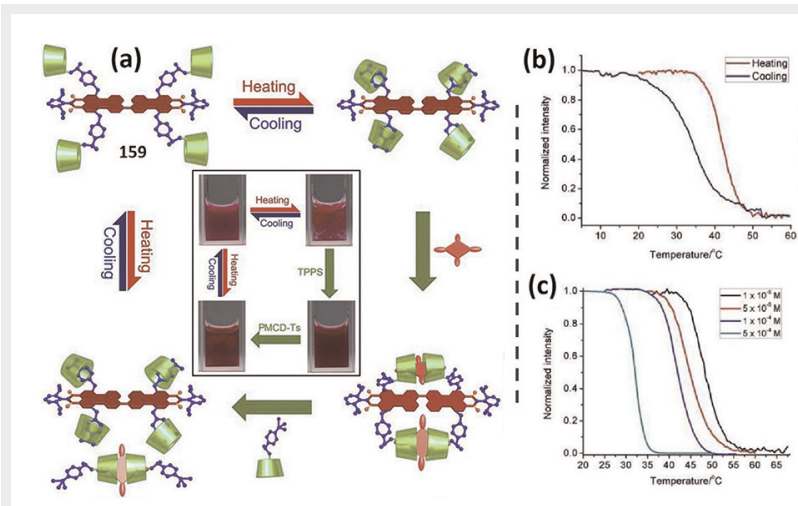

Figure 36 Bi-PMI derivative with permethyl- $\beta$-cyclodextrin grafts (BPMI-CD) showing a host-guest controlled thermo-responsive behavior. Reversible LCST behavior of BPMI-PMI (159) in water (a) under heating and cooling cycles, and (b) concentration-dependent behavior observed at $700 \mathrm{~nm}$. Reprinted with permission from Ref. 61, Copyright 2019 Elsevier.

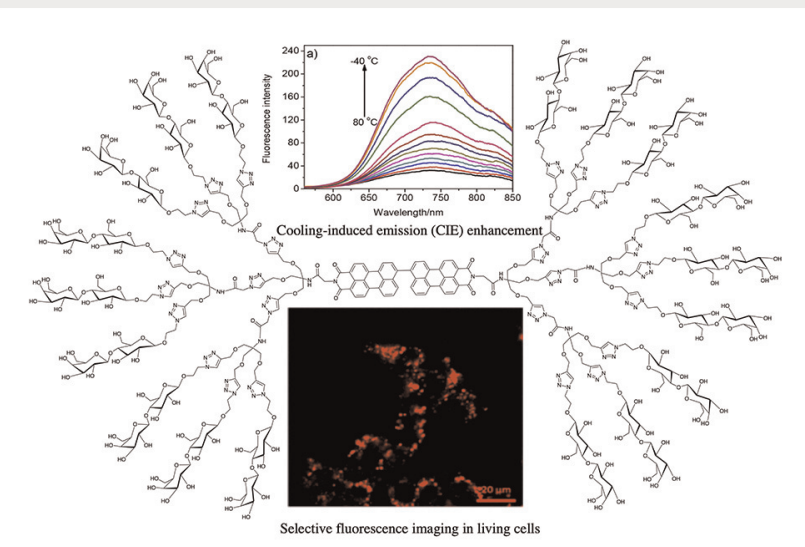

Figure 37 Structure of BPMI-18Lac (103) applied for cooling-induced emission (CIE) enhancement and live-cell fluorescence imaging. Reprinted with permission from Ref. 62 Copyright 2019 American Chemical Society.

hancement was associated with the inhibition of the intramolecular electron interactions between the PMI moieties linked by the $\mathrm{C}-\mathrm{C}$ single bond. Additionally, BPMI-18Lac showed selective fluorescence imaging for HepG 2 cells through the asialoglycoprotein receptor (ASGP-R) on the cell surface (Figure 37, bottom part).

\section{Photo-Responsive PMI Derivatives}

There are only a few examples of photochromic materials made from PMI as a chromophore. Zhu and colleagues reported a PMI dyad modified with an increasing number of
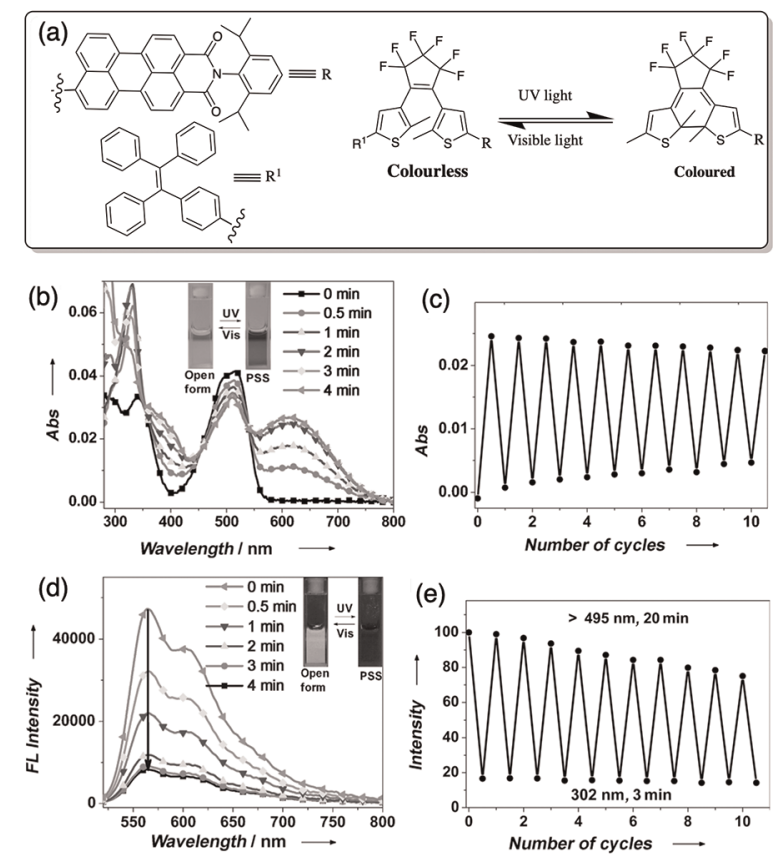

Figure 38 (a) Chemical structure of photoswitchable donor (TPE)photochromic bridge-(DTE)-acceptor (PMI) conjugates. (b) Absorption spectra of TPE-DTE-PMI (114) in toluene upon irradiation with UV light $(302 \mathrm{~nm})$. The photostationary state (PSS) was determined by irradiating a solution of TPE-DTE-PMI with UV light until no changes were observed in the spectrum. The inset shows photos of TPE-DTE-PMI in toluene upon irradiation with visible and UV light. (c) Reversible absorption switching for TPE-DTE-PMI in toluene measured at $620 \mathrm{~nm}$ upon alternating irradiation with UV and visible light. (d) Emission spectrum change of TPE-DTE-PMI in toluene upon irradiation with UV light $(302 \mathrm{~nm})$. The inset shows photos of TPE-DTE-PMI in toluene taken at $365 \mathrm{~nm}$ UV light after irradiation with visible light and UV light, respectively. (e) Reversible fluorescence switching for TPE-DTE-PMI in toluene measured at $567 \mathrm{~nm}$ $\left(\lambda_{\mathrm{ex}}=514 \mathrm{~nm}\right)$ upon alternating irradiation. Reprinted with permission from Ref. 63a. Copyright 2014 Wiley-VCH.

dithienylethene (PMI-nDTE, $n=1-3$ ) units (Figure 38a). ${ }^{63}$ Later, they have developed recyclable fluorescence patterning and an all-optical transistor using highly sensitive on-off fluorescence quenching associated with the photochromic properties of DTE. The photochromic fluorescence quenching ratio of PMI-3DTE (139) in the photostationary state exceeds 10,000 and the fluorescence quenching efficiency is close to $100 \%$ with few seconds of UV radiation (Figure 38b,c). ${ }^{63 a}$ The photochromism and fluorescence switching properties were investigated in a poly(methyl methacrylate) film and in toluene (Figure 38d, e). ${ }^{63 a}$ The fluorescence is reversibly quenched based on photochromic fluorescence resonance energy transfer from PMI to DTE units upon conversion between the open and close-ring isomeric forms upon sequential irradiation of UV and visible light. Further, the 

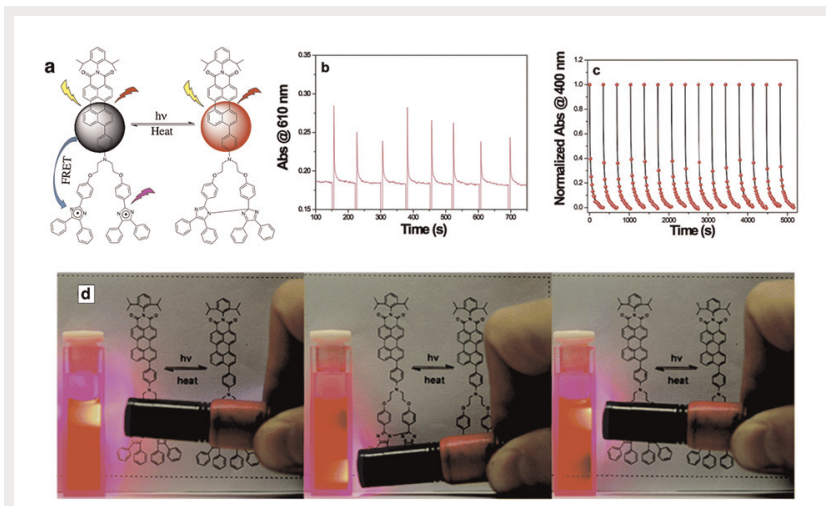

Figure 39 (a) Structure and photoswitching property of PMI-hexaarylbiimidazole (PMI-N-HABI, 136). Fast photoswitching fading kinetics of PMI-N-HABI monitored at (b) $610 \mathrm{~nm}$ and (c) $400 \mathrm{~nm}$. (d) Photos of $81 \mu \mathrm{M}$ PMI-N-HABI in toluene upon excitation by an UV laser pen. Reprinted with permission from Ref. 65. Copyright 2016 Royal Society of Chemistry.

switching property of PMI-3DTE was utilized for nanoimaging of block copolymer vesicles in high resolution. The same concept of photo-switchable properties of PMI-2DTE (169) $^{63 \mathrm{~b}}$ functionalized with a PEG (PEG-PMI-2DTE) was used further for super-resolution imaging of liposomes with an optical resolution of $30 \mathrm{~nm}$.

Later, the same group has designed a water-soluble, photoswitchable polymeric probe (P4) for fluorescence imaging with a super-resolution microscopic technique. ${ }^{64}$ The PMI2DTE molecule was conjugated with a hydrophilic $N$-isopropylacrylamide polymer at the peri-position in combination with two-fold mitochondria-targeting units triphenylphosphonium and vinylbenzyl chloride (170). The selective colocalization of $\mathbf{P 4}$ to mitochondria was established with the commercially known mitoTracker green dye. The strong fluorescence properties of $\mathbf{P 4}$ with desirable fluorescence switching behavior in combination with selective mitochondria targeting enable super-resolution imaging of sub-cellular structure with sub-30 $\mathrm{nm}$ spatial resolution in imaging subcellular organelles (vide infra).

Zhu and co-workers have developed the donor-photochromic bridge-acceptor (D-P-A) triad, in which tetraphenylethene (TPE) acts as the electron donor, PMI as the electron acceptor, and DTE as the photochromic bridge. ${ }^{65}$ The authors have investigated the absorption, emission, photochromism, and fluorescence photoswitching behavior of the TPE-DTE-PMI system in solution, in aggregates, and in the solid state. In both solution and solid films, this system shows a fluorescence quenching upon UV irradiation resulting from photochromic fluorescence resonance energy transfer. For TPE-DTE-PMI, excellent color and fluorescence switching properties were visible for multiple cycles.
In another report, Zhu and co-authors designed and synthesized a fluorescent molecular switch based on PMIhexaarylbiimidazole dyad (PMI-N-HABI, 136). ${ }^{65}$ PMI-NHABI (136) exhibits a photoswitchable NIR fluorescence (Figure 39a) along with a photoswitchable electron paramagnetic resonance (EPR) resulting from photo-induced twin radicals. The radical nature of the colored species is assessed by EPR spectroscopy. The visible color of PMI is switched between two states as shown in Figure 39(b, c). The emission could also be reversibly quenched by the photo-induced radicals through the FRET mechanism. Further, the fluorescence-switching properties (Figure 39d) of PMI$\mathbf{N}$-HABI are applied to the optical nano-imaging of polymer nanowires.

\section{Electrochemical Properties of Rylene Derivatives}

The redox properties of the rylene derivatives are very important for their applications as organic materials. Here, we wanted to provide a brief comparison on how the introduction of imide group, number of naphthalene units, and incorporation of donor and acceptor group influences their electrochemical properties. It is well established that the variation at the imide position does not change the reduction potential. In $\mathrm{CH}_{2} \mathrm{Cl}_{2}$, NMI has a reduction potential of $-1.50 \mathrm{~V}$. However, the incorporation of another imide group at the peri-position of NMI results in more electron-deficient naphthalenediimide (NDI) which has a reduction potential value of $-1.13 \mathrm{~V}$ (as shown in Figure 40). Similarly, PMI has a reduction potential value of $-1.46 \mathrm{~V}$, and the incorporation of another imide group at the peri-position in the case of PDI reduces its reduction potential by $0.40 \mathrm{~V}$. Interestingly, the introduction of a donor group at the peri-position of PMI did not have much influence on the reduction potential. ${ }^{56}$ However, when an electron-withdrawing group $\left(-\mathrm{CF}_{3}\right)$ was incorporated in the peri-position of PMI, the reduction potential drastically reduces to $-1.30 \mathrm{~V}$ and further addition of $-\mathrm{CF}_{3}$ group at the bay-position further reduces the potential to $-1.01 \mathrm{~V} \cdot{ }^{40}$

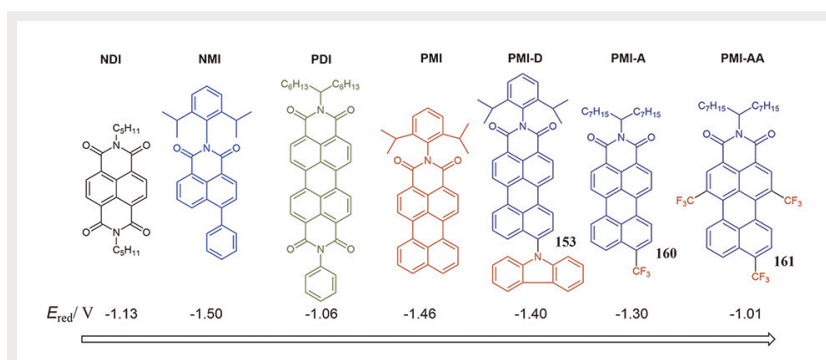

Figure 40 Modulation of reduction potential by incorporation of electron-donating and electron-withdrawing groups in the rylene family. 


\section{Self-Assembling Properties of PMI Derivatives}

Supramolecular self-assembly primarily circumvolves around chemical systems constructed from assembled molecular subunits fastened together by non-covalent interactions. Supramolecular self-assembly paves the pathway to fabricate well-organized functional nanomaterials which can help bridge the gap between natural and artificial systems. Resorting to non-covalent interactions such as $\mathrm{H}$ bonding, dipole-dipole interaction, $\pi-\pi$ stacking, van der Waals force, hydrophobic effect, electrostatic interaction and metal-ligand coordination aids in the construction of supramolecular assemblies. In most cases, intrinsic $\pi-\pi$ stacking and directional $\mathrm{H}$-bonding play the predominant role in self-assembly processes. Perylene imides are an obvious choice to pursue self-assembly studies owing to the large $\pi$-surface area that triggers $\pi-\pi$ stacking. ${ }^{66}$ Although PMI exhibits similar self-assembling propensity as its sister analogue PDI, its self-assembling property has hitherto remained elusive. Nonetheless, several scientists have been instrumental in exploring the self-assembling of PMIs and their applications in ultrafast charge separation, light harvesting arrays, stimuli-responsive assemblies, white light emission and supramolecular weave.

Wasielewski's group in 2012 explored the self-assembly and ultrafast electron transfer dynamics of a PMI covalently bonded to an $N, N$ '-(bis-tridodecyloxyphenyl)melamine inserting biphenyl as the spacer unit between them (Figure 41a). ${ }^{67}$ Small-angle X-ray scattering/wide-angle X-ray scattering (SAXS/WAXS) data show that monomers spawn hydrogen-bonded foldamers in methylcyclohexane $(\mathrm{MCH})$ with the aid of $\pi-\pi$ stacking and multipoint hydrogen bonding. While the monomeric form was reluctant to show any PET in solution, the hydrogen-bonded foldamer exhibited fast charge separation dynamics $\left(\tau_{\mathrm{cs}}=3.4 \mathrm{ps}\right)$ in $\mathrm{MCH}$. The slower charge recombination rate within the foldamer was found to be in good correlation with a two-turn helix model

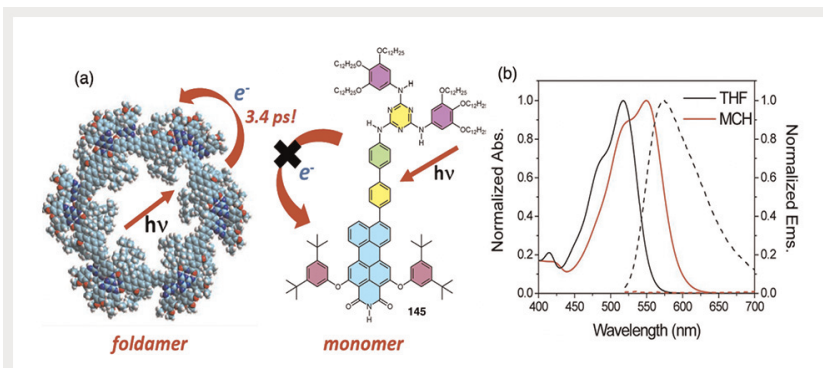

Figure 41 (a) Overview of the self-assembly process occurring in 145. (b) Normalized absorption and emission spectra of 145. Reprinted with permission from Ref. 67. Copyright 2012 American Chemical Society. proposed by the authors. In $\mathrm{MCH}$, self-assembly leads to a $33 \mathrm{~nm}$ shift in the absorption maxima relative to its monomer in THF (Figure 41b), while the fluorescence intensity was completely quenched in $\mathrm{MCH}$ on account of electron transfer operative within the self-assembled foldamer. This contribution thus delineates the role of self-assembly in tuning the photophysical properties of PMI-based chromophores.

The same group in 2014 synthesized two symmetric PMI trefoils in which PMI is attached through the 9-position to either the 1,3,5-positions of a benzene ring (134, Figure $42 \mathrm{a}$ ) or the 4-positions of the phenyl rings in 1,3,5-triphenylbenzene (135, Figure $42 \mathrm{~b}){ }^{42}$ Both of these molecules displayed monomeric nature in THF, which were converted to self-assembled structure in MCH. In THF, both 134 and 135 exist as monomers evident from the appearance of an intense $(0,0)$ absorption band accompanied with a slightly weaker $(0,1)$ vibronic band. In $\mathrm{MCH}$, both 134 and 135 exist in a self-assembled state as evident from the inversion in the intensities of the vibronic bands. This inversion of band intensities arises from the excitonic coupling of the transition dipole moments of adjacent PMI chromophores hinting towards the possible formation of an H-aggregate (Figure 42). Incorporation of a phenyl spacer between the PMIs and the central benzene ring induces planarity in 135 leading to an enhanced excitonic coupling compared to $\mathbf{1 3 4}$ that is well reflected in their absorption spectra. In corroboration with the above spectroscopic observation, SAXS/WAXS data reveal that the planarity of $\mathbf{1 3 5}$ induces greater extent of selfassembly to form tridecamer, whereas the self-assembly in (a)
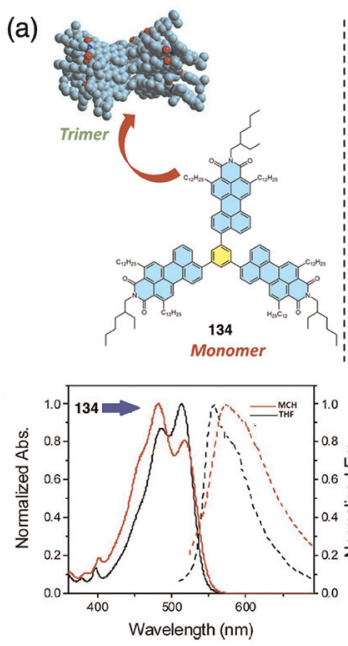

(b)
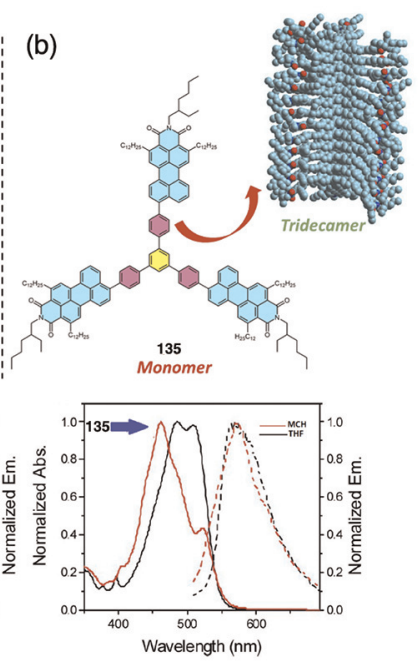

Figure 42 (a) Representation of the self-assembled structures fabricated from 134 and 135; (b) normalized absorption and emission of 134 and 135. Reprinted with permission from Ref. 42. Copyright 2014 American Chemical Society. 
134 is restricted to trimers. The steady-state fluorescence of monomeric 134 and 135 in THF displays vibronically resolved features, whereas in $\mathrm{MCH}$ the self-assembled structure of 134 and 135 results in a bathochromically shifted broad emission. Moreover, the QYs are drastically quenched in $\mathrm{MCH}$. These distinct steady-state emission features observed in THF and $\mathrm{MCH}$ are indicative of excimer formation, which has been validated through time-resolved spectroscopy.

Stupp's group in 2019 developed a PMI-based stimuli-responsive amphiphilic supramolecular photosensitizing assembly. ${ }^{68}$ For this purpose, they synthesized an amphiphilic hydroxylated PMI (120, Figure 43a) which dissolved in water containing 2 equiv. $\mathrm{NaOH}$ to yield a deep blue solution. The solution was rendered reddish-brown on addition of 1 equiv. $\mathrm{HCl}$. These color changes occurring in response to acid or base are notified by a shift of $187 \mathrm{~nm}$ in the absorption spectrum. In order to confirm whether the ionisation of the hydroxyl group leads to the $\mathrm{pH}$-dependent observed color change, the authors explored an analogous compound embellished with a bulky imide substituent (162, Figure 43b) which remains in the monomeric form in organic solvent. This molecule also registers a drastic change of red to blue color upon addition of a base, demonstrated by a $133 \mathrm{~nm}$ shift in the absorbance spectrum (Figure 43c,d).

Interestingly, the shapes of the absorption spectra differ drastically under the influence of $\mathrm{pH}$. This led the authors to speculate that apart from the ionisation state of the hydroxyl group, the distinct self-assembly state adopted in acidic and basic conditions registers a drastic change in their absorption spectrum both in terms of line shape and absorption maxima. In WAXS, 162 revealed sharp scattering peaks suggesting a higher degree of order within the nanostructures. Cryo-TEM data (Figure 43e,f) showed that 162

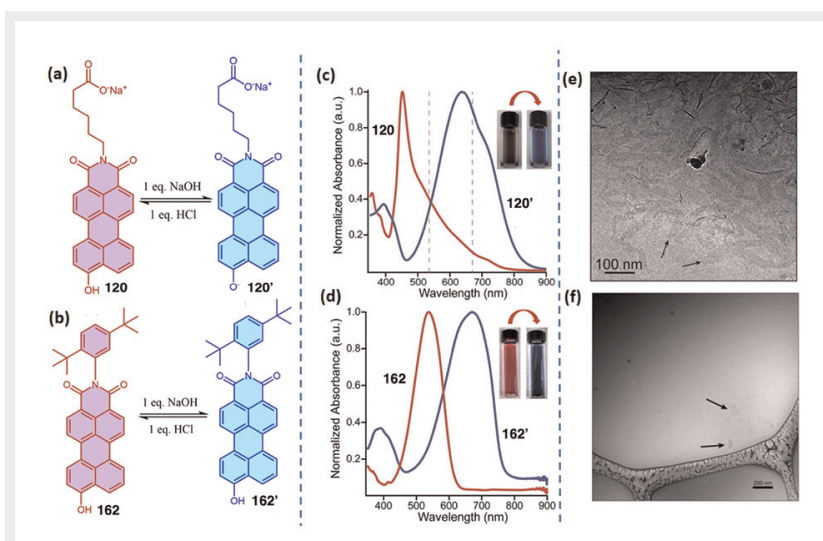

Figure 43 (a) Structure, (c) change of absorbance on deprotonation and (e) cryo-TEM image of 120. The same order of representation, (b), (d), and (f), was followed for 162. Reprinted from Ref. 68 published under a creative commons license (CC BY). indeed formed supramolecular nanoscale ribbons while 162' gave rise to only small aggregates consistent with the appearance of a single wide, less intense scattering peak in WAXS. The authors suggested that the addition of a second negative charge to the PMI core after deprotonation (162') thwarted the formation of crystalline nanoribbons as observed for $\mathbf{1 6 2}$. $\mathrm{CaCl}_{2}$ was used to screen electrostatic repulsion among molecules. When exposed to $\mathrm{CaCl}_{2}$, the absorbance of 162' becomes significantly blue-shifted bearing resemblance to the absorption spectrum of $\mathbf{1 6 2}$. However, the absorption spectrum of $\mathbf{1 6 2}$ remains unperturbed under the influence of $\mathrm{CaCl}_{2}$. Thus, it can be conceived that electrostatic repulsion hinders the formation of ordered nanostructures and supramolecular crystallization can be induced only in the presence of strong screening ions. The authors also found that the electronic energy levels (HOMO/LUMO) of this supramolecular nanostructure are altered in response to change in $\mathrm{pH}$. The assemblies were further utilized as dynamic photosensitizers (PSs) in both acidic and basic media. Using $\left[\mathrm{Mo}_{3} \mathrm{~S}_{13}\right]^{2-}$ as the catalyst, $\mathrm{H}_{2}$ was generated at a $\mathrm{pH}$ of 4 , whereas using an iron-porphyrin catalyst, protons could be reduced to hydrogen and $\mathrm{CO}_{2}$ was reduced to $\mathrm{CO}$.

Our group has also reported self-assembly arising from an alkyne-phenanthrene-substituted PMI, PMIAP (34), that gives rise to an intermediate white light emission via the formation of fluorescent organic NPs. ${ }^{69}$ Time-dependent emission of PMIAP carried out in THF showed that with the increase of measurement time, a drastic change in emission spectra occurred (Figure 44a). The initially yellow fluorescent solution of PMIAP in THF changed to cyan within 10$12 \mathrm{~h}$ via an intermediate white light emission at about $6 \mathrm{~h}$ (Figure 44b). The color change ratified the switching in emission peak from 570 to $465 \mathrm{~nm}$ (Figure 44c). The authors have cogitated that the aforementioned emission change transpires on account of its aggregation in THF. Time-dependent scanning electron experiments divulge that budding initiates from the initially formed fiber-like network to finally give rise to spherical NPs (Figure 44e). The NPs undoubtedly arise due to aggregation, the PMI forming the dark core, whereas the appended phenanthrene forms the fluorescent fringe. Once the aggregation proceeds to yield NPs, the intramolecular electronic communication between the PMI core and phenanthrene is truncated and they behave as two individual chromophores (Figure 44d). This results in the intense emission band of the phenanthrene moiety at $465 \mathrm{~nm}$, thus ruling out the possibility of energy transfer to the acceptor perylene core. Hence, a near-orthogonal orientation of the two chromophores is suggested, devoid of any intramolecular electronic conjugation. The authors have also found that the assembled NPs are extremely robust and can endure the effect of sonication and reflux. Hence, they can be easily dried and dissolved in other solvents. Therefore, the white light could be easily engendered 

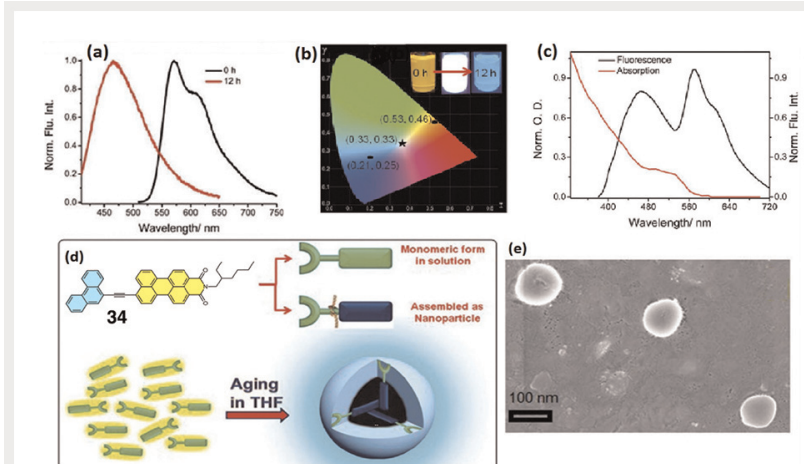

Figure 44 (a) Time-dependent emission of 6 in THF; (b) images of time-dependent emission and chromaticity coordinates of 6 in THF; (c) absorption and emission spectra of the white light emission of 6; (d) schematic representation of the plausible self-assembly in 6; (e) SEM image of the nanoparticles of 6 . Reprinted with permission from Ref. 69. Copyright 2017 Royal Society of Chemistry.

by mixing the monomeric and NP solution in a good solvent or at an intermediate time.

Working on the same line, our group has also explored the dynamic self-assembly of a PMI-pyrene conjugate PMIAPy (138) ${ }^{70}$ The orange fluorescent nascent solution of PMIAPy in dry THF metamorphosed to a cyan fluorescent solution upon aging accompanied with an intermediate white light emission (Figure 45a). The color change well corroborates with the change in the emission band from $605 \mathrm{~nm}$ to $470 \mathrm{~nm}$. The authors have speculated that such a prominent change in emission band stems from the dynamic self-assembly of PMIAPy in THF. Time-dependent scanning electron microscopy (SEM) experiment of PMIAPy in THF (Figure 45b-g) evinces the formation of five distinct morphological phases in the event of NP formation. The pyrene moiety forms a pre-associated excimer in the ground state which induces greater non-linearity into the hitherto non-coplanar PMIAPy. Truncation of electronic conjugation occurs along with formation of weakly fluorescent $\mathrm{H}$-aggregates from the perylene segments. Loss of linearity chaperoned with $\mathrm{H}$-aggregate formation quenched the lower energy band and ingenerated the loss of energy transfer between the donor pyrene and acceptor perylene (Figure $45 \mathrm{~h}$ ). The NPs formed were robust and hence were ingeniously applied by the authors for white light emission upon mixing the monomer and NP solution in a $1: 3$ volume ratio.

Wennemers' group in 2017 discovered a robust triaxial supramolecular weave knitted from an oligoproline PMI conjugate (115, Figure 46a). The supramolecular aggregate formed by the conjugate was inspected by transmission electron microscopy (TEM) which revealed the presence of a mesh-like superstructure composed of triangles and hexagonal holes (Figure 46b). ${ }^{71}$ Grazing incidence wide-angle $\mathrm{X}$-ray scattering (GIWAXS) explicitly unraveled the molecu-
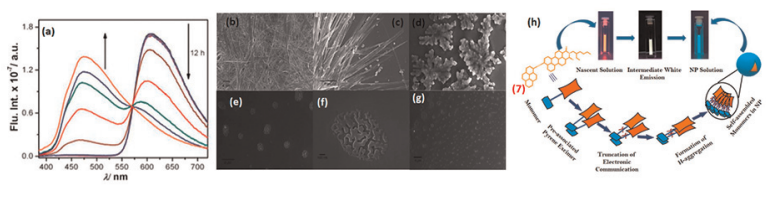

Figure 45 (a) Time-dependent emission of 115 in THF; time-dependent SEM image of 115 in THF monitored for (b) nascent solution, (c) $1 \mathrm{~h}$, (d) $3 \mathrm{~h}$, (e) $10 \mathrm{~h}$, (f) zoomed assembly after $10 \mathrm{~h}$, and $(\mathrm{g})$ unyoking of nanoparticles in THF; (h) possible mechanistic pathway of the selfassembly process of $\mathbf{1 1 5}$. Reprinted with permission from Ref. 70 . Copyright 2018 Royal Society of Chemistry.

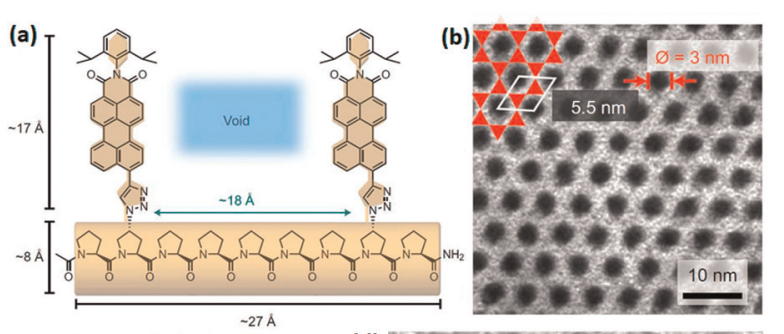

(c)

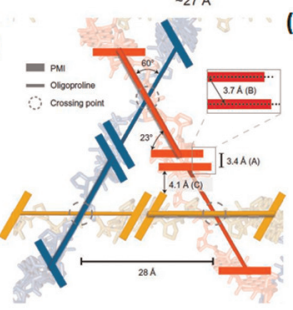

(d)

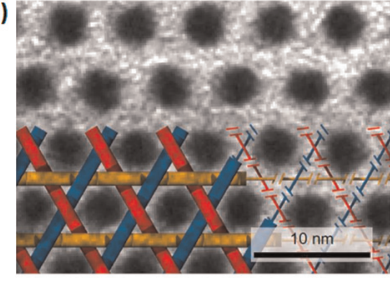

Figure 46 (a) Structure of the conjugate 115; (b) TEM image of the trihexagonal superstructure formed from 115; (c) possible arrangement of the chromophores of $\mathbf{1 1 5}$ derived from GIWAXS data; (d) illustration of the molecular organization of the superstructure formed from 115 superimposed on TEM micrograph. Reprinted with permission from Ref. 71. Copyright 2017 Springer Nature.

lar arrangement of the conjugate within the superstructure. The oligoproline-PMI conjugate self-assembles predominantly through $\pi-\pi$ interactions to form $\pi$-stacked chromophores perpendicular to the $\mathrm{Si} / \mathrm{SiO}_{2}$ surface. Additionally, weak $\mathrm{CH}-\pi$ interactions between the $\pi$-stacked PMI pairs concoct the triangular structure of the connection points within the network which inseminates to the trihexagonal pattern of the self-assembled superstructure (Figure 46c, d). Their finding thus widened the purview of complex supramolecular architecture at the molecular level.

\section{Applications of PMI in Solar Cells}

Fossil fuel resources have steadily depleted over the years in trying to keep up with the escalating demand for energy 
supply and are on the brink of exhaustion. The current reserve of fossil fuels could only last 40 years for oil, 60 years for natural gas and 200 years for coal. ${ }^{72}$ Thus, the impending decline of conventional non-renewable energy resources has propelled advancement in alternative renewable technologies. Over the years, renewable energy resources such as solar, hydropower, biomass, wind and geothermal have evolved as sustainable energy reserves. Among the aforementioned renewable energy sources, photovoltaic technology has captured immense adoration for harvesting solar energy to generate electrical energy. Conventional siliconbased solar cells suffer from high cost, low efficiency and being environmentally perilous. Hence, they have been progressively replaced by their organic and hybrid counterparts, popularly known as OSCs and DSSCs. The organic and hybrid counterparts have emerged as an obvious choice for photovoltaics owing to their cost-effectiveness, high PCE and simple fabrication mode. Thanks to the pioneering and ground-breaking discovery of molecular photovoltaics by O'Regan and Gratzel, who conceived and realized that photo-systems based on molecular light harvesters can rival and even outperform conventional solar cells. ${ }^{73}$ This seminal work by Gratzel and co-workers spurred the scientific minds to develop organic architectures that can either serve as the light-absorbing layer in OSCs or as sensitizers in DSSCs. The high molar extinction coefficients combined with the possibility of molecular engineering make the organic dyes a promising candidate for photovoltaic applications. In the flagship family of $\pi$-conjugated organic molecules, perylene imides (PDIs and PMIs) represent one of the extensively delved classes of organic n-type semiconductors having robust thermal, chemical and photostability combined with intense absorption in the UV-Vis. region, ease of structural tunability, excellent charge transport property and high fluorescence QY. The aforementioned lucrative properties make perylene imides an obvious choice in photovoltaics giving it an edge over other state-of-the-art organic dyes. PDIs have already been widely used in flat heterojunction solar cells whilst their asymmetric analogue PMIs have found wide acceptability in bulk heterojunction solar cells as well as DSSCs. ${ }^{74}$

\section{PMls in Bulk Heterojunction Solar Cells}

Bulk heterojunction solar cells consist of an active layer wherein the donor and acceptor units are dispersed into each other to facilitate effective photon absorption (as shown in Figure 47). ${ }^{75}$ The foremost criterion for any material to be used in bulk heterojunction is their self-assembling propensity. In this regard, the PMIs although less symmetrical compared to their parent PDIs exhibit a similar self-assembling tendency mainly predominated by $\pi-\pi$ interactions and hence prove conducive to be used as acceptor ma-

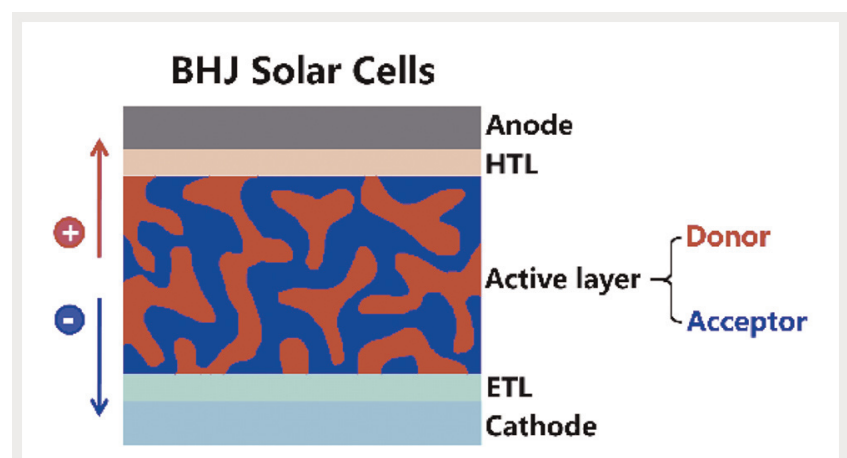

Figure 47 Representation of a bulk heterojunction solar cell. ${ }^{75}$

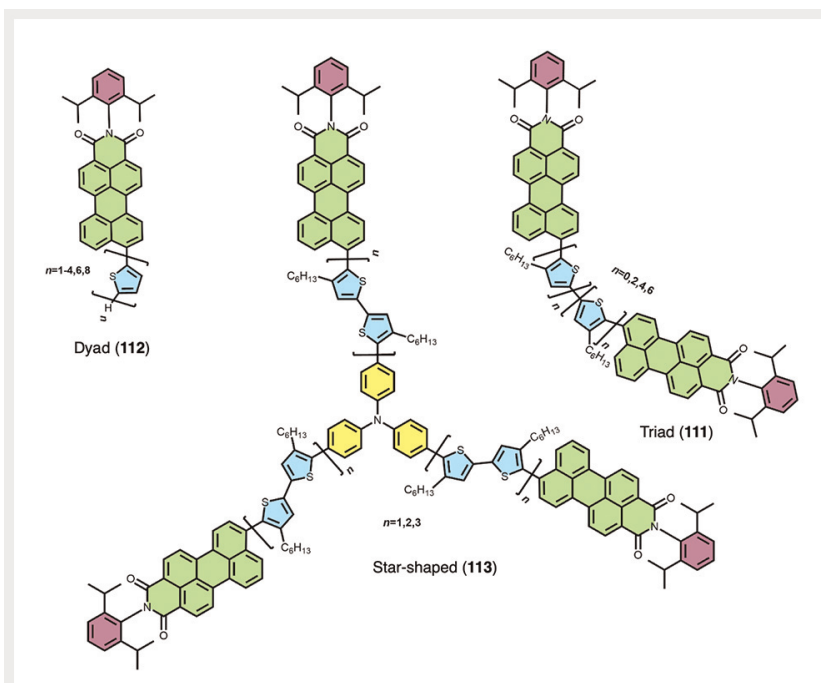

Figure 48 PMI-oligothiophene systems used in bulk heterojunction solar cells. ${ }^{76}$

terials in bulk heterojunction solar cells. Although PMIs are endowed with less absorption coefficients and higher LUMO energies compared to their parent PDIs, nevertheless they have been used in several bulk heterojunction devices. In these devices, a donor-acceptor coupled system has been utilized wherein PMI serves as the acceptor and thiophene acts as the donor. Dyads, triads and star-shaped donor-acceptor system (111-113, Figure 48) have emanated employing PMI and thiophene. This PMI-thiophene combination is very illustrious in photovoltaics as the PMI moiety absorbs highly in the visible region, and thiophenes exhibit exemplary charge transport properties and inflate absorption at lower wavelengths. Moreover, both of these moieties evince remarkable self-assembling properties enabling them to be fabricated into thin films. The PCEs achieved with these PMI-oligothiophene concoction spans from $0.2 \%$ for triad, $0.25 \%$ for star-shaped structure to $0.48 \%$ for the dyad. Thus, it can be realized that the PCEs achieved in bulk heterojunc- 

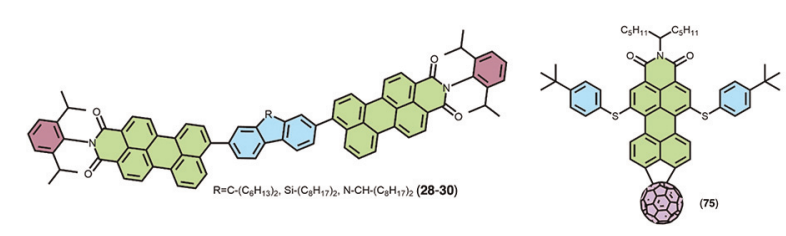

Figure 49 PMI-based non-fullerene acceptors used in bulk heterojunction solar cells.

tion devices using PMI as an acceptor are yet to cope up with the performance exhibited by donor-acceptor copolymers that have demonstrated PCEs up to $8.3 \%$.

PMI-based non-fullerene acceptors are often used in conjunction with various donor moieties in bulk heterojunction solar cells. In this regard, Trimmel's group in 2020 designed and synthesized various PMI-linker-PMI based acceptors. ${ }^{30}$ Such a design strategy introduces significant twisting into the system and provides good solubility by overcoming the $\pi-\pi$ stacking. These three fluorene derivatives (28-30, see left part of Figure 49 for their structures) in concurrence with PBDB-T as a donor furnished a maximum device efficiency $(\eta)$ of $5.16 \%$.

Wang's group in 2020 synthesized a bay-decorated PMIfullerene hybrid acceptor (75, see Figure 49) to boost up the PCE. ${ }^{37}$ Their perception was materialized in the achievement of a ground-breaking PCE of $8.01 \%$.

\section{PMIs in Dye-Sensitized Solar Cells}

The pre-eminent application of PMIs in photovoltaics can be discerned in DSSCs where they are amongst the most efficient sensitizers. A DSSC (schematically shown in Figure 50) is a photo-electrochemical system where an organic dye photosensitizes a metal-oxide anode for producing electricity. ${ }^{77}$

Most PMI-based organic sensitizers employ a push-pull design strategy, i.e. coupling of a strong electron donor and an acceptor so as to ensure orbital partitioning of HOMO and LUMO to engender strong intramolecular dipole. Such a designing approach not only facilitates (i) electron injection from the LUMO of the photoexcited dye to the conduction band of the semiconductor metal oxide but also (ii) restores the dye to its ground state by allowing electron transfer from the hole-transporting material to the HOMO of the dye. This push-pull strategy results in a significant broadening of the absorption spectra of the dye which is advantageous for light-harvesting application.

Different PMI sensitizers will be discussed below and will be grouped according to their common designing approaches (as shown in Figure 51) as enlisted below:

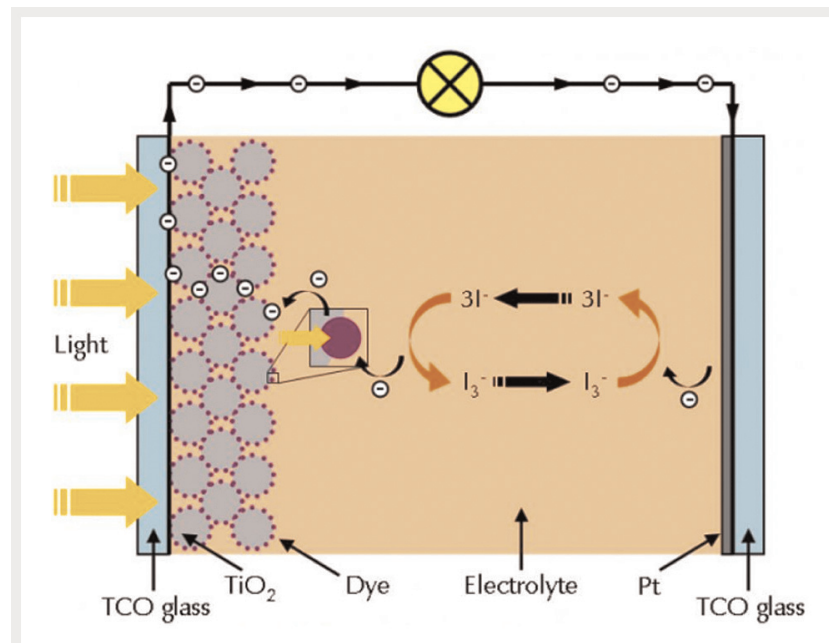

Figure 50 Working principle of a dye-sensitized solar cell. ${ }^{78}$

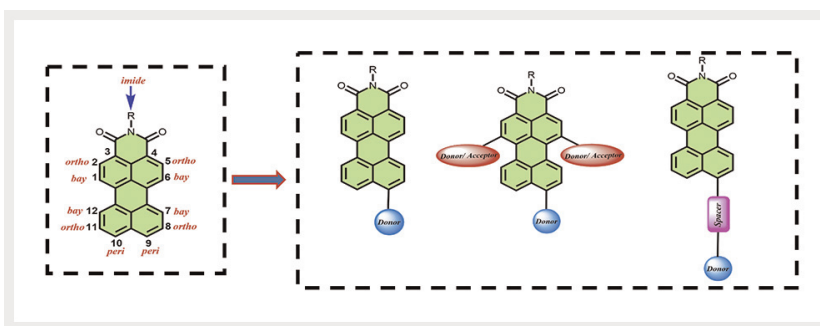

Figure 51 Design principles of PMI sensitizers used in DSSCs. ${ }^{79}$

(i) PMIs bearing directly attached donor moiety in the periposition.

(ii) PMIs with additional bay-functionalization in addition to peri-substitution.

(iii) PMIs with a spacer moiety introduced between the perylene core and the peri-substituent.

\section{(i) PMIs bearing directly attached donor moiety in the peri- position:}

Majority of the push-pull PMIs bear an alkyl or aryl amine as the donor (see Figure 52). A few such examples are documented below.

In both the molecules shown in Figure 52, the carboxylic acid group is introduced at the imide position to enable it to anchor on the $\mathrm{TiO}_{2}$ surface. In 124, a swallow tail disposed long chain aliphatic amine was used as the donor, while in 125 a diphenylamine with branched alkyl chains was utilized as the donor. This kind of branched alkyl chains are more sterically encumbered compared to the linear analogues thereby providing shielding towards the hole-transporting material. The phenyl groups in $\mathbf{1 2 5}$ serves to enlarge the conjugated system as well as suffice to introduce considerable twisting for orbital partitioning of HOMO and LUMO. Both 124 and 125 achieve a photoconversion efficiency $(\eta)$ 

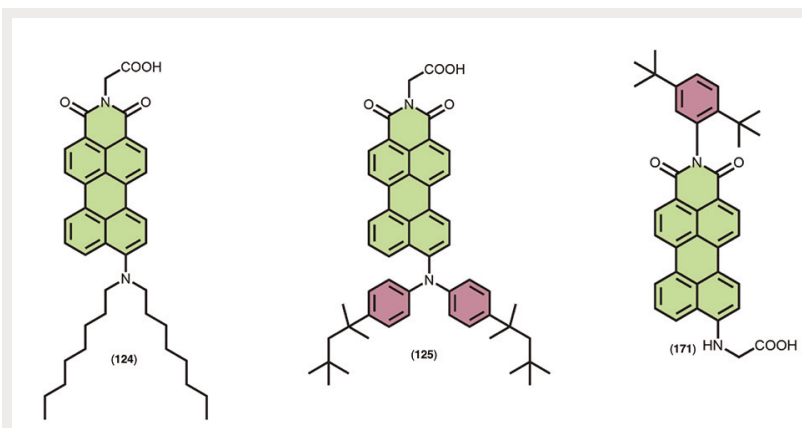

Figure 52 Peri-functionalization-based PMI sensitizers. ${ }^{80}$

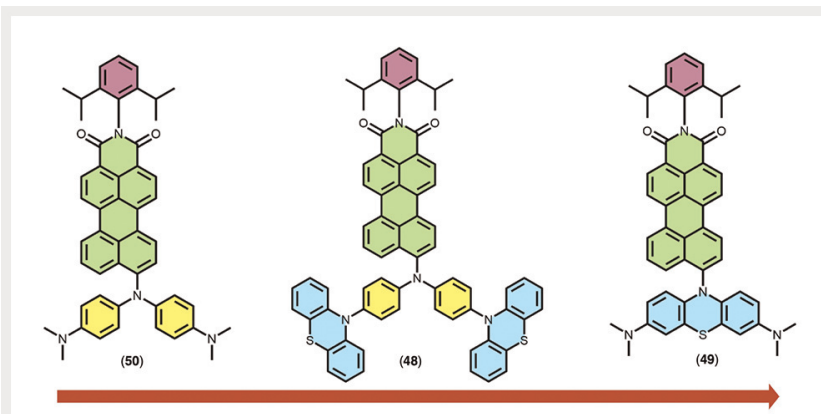

Figure 53 Peri-functionalization-based rainbow PMI sensitizers. ${ }^{33}$

of $1.2 \%$ in liquid cells but $\mathbf{1 2 5}$ exhibits thrice raised $\eta$ of $3.2 \%$ in solid-state cells. Additionally, PMI 125 has emerged as the most extensively investigated PMI sensitizer till date. PMI 171 exhibits a similar photovoltaic performance to 124 , but its design concept resembles that of a reversed sensitizer.

Introduction of sulphur as a heteroatom in addition to nitrogen has also been utilized for peri-functionalization (as shown in Figure 53). All of the aforementioned dyes (48, 49, 50) are classical push-pull systems showing different degrees of orbital partitioning. This orbital partitioning mainly results from the different degrees of twisting induced into the system by various donors. Besides, these sensitizers are also susceptible to color tuning as their absorption maxima reported are 668, 548 and $515 \mathrm{~nm}$ in DCM, respectively. The authors speculate that the ease of color tunability and the broad absorption of these dyes qualify them as sensitizers.

(ii) PMIs with additional bay-functionalization in addition to peri-substitution:

An improved approach in sensitizer design involves bayfunctionalization in addition to peri-functionalization (see Figure 54 for structures). This approach sterically occludes the aggregation proclivity of the perylene core which drastically reduces the unwanted recombination reactions between the dye molecules. PMI (149) has been decorated with phenylthio groups in the 1,6 (bay) positions and PMI

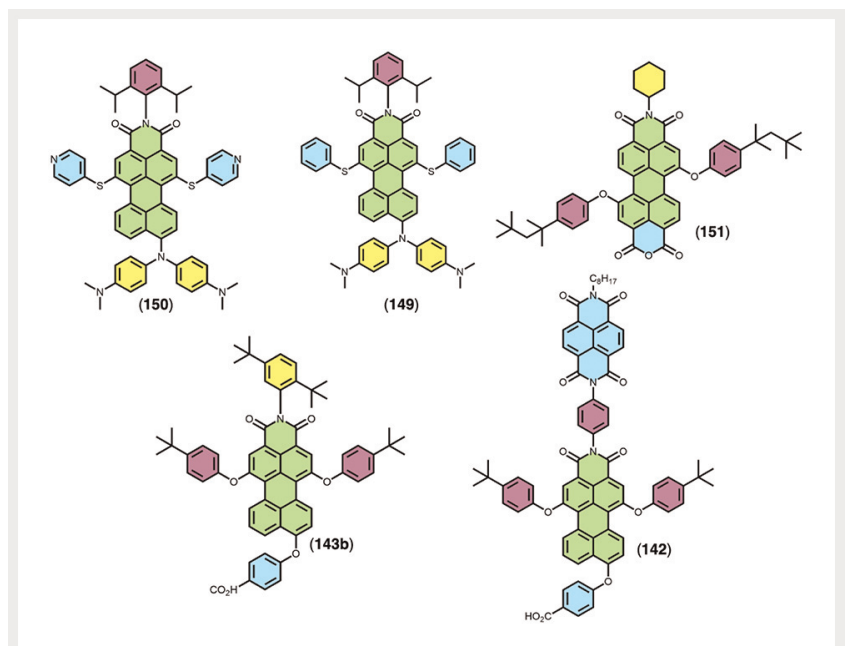

Figure 54 Bay-functionalization-based PMI sensitizers. ${ }^{33,81}$

(150) has pyridylthio groups in the same position. These groups not only hinder aggregation of the planar perylene core but are also capable of fine-tuning the optical properties of these sensitizers. PMIs 149 and 150 exhibit bathochromically shifted absorption maxima at $681 \mathrm{~nm}$ and $736 \mathrm{~nm}$ respectively attributed to their bay-substituents. For PMIs 151 and 143b, phenoxy substituents were introduced as bay-substituents to restrict aggregation and tune their electrochemical properties. PMIs 143b and 142 are classic examples of reversed sensitizers used in p-type solar cells. In reversed sensitizers, the anchoring group used for binding to the semiconductor is close to the donor moiety rather than being proximate to the acceptor. The imide hydrogen of PMI 143b is substituted with di-( $t$-butyl) phenyl while a NDI tenants the imide position in PMI 142 rendering a dyad system. While PMI 143b achieves a PCE value of $1.3 \%$, the PMI-NDI dyad system exhibits an enhanced PCE value of $4 \%$ as well as a longer charge separation lifetime. The decoupled HOMO-LUMO of the dyad is instrumental for such photovoltaic performance of PMI 142.

\section{(iii) PMIs with a spacer moiety introduced between the perylene core and the peri substituent:}

Another approach of fabricating PMI-based sensitizers involves the insertion of a spacer moiety between the PMI core and the peri-substituent (as shown in Figure 55). PMI sensitizer $172(n=3)$ consists of a bridging oligothiophene group spacer of varying length between the PMI acceptor and triphenylamine acceptor. As the length of the oligothiophene spacer increased, it led to an incremental decoupling of the HOMO and LUMO. This proves particularly essential for attaining spatial separation of charge carriers for curtailing the possibility of charge recombination. Undoubtedly, the 


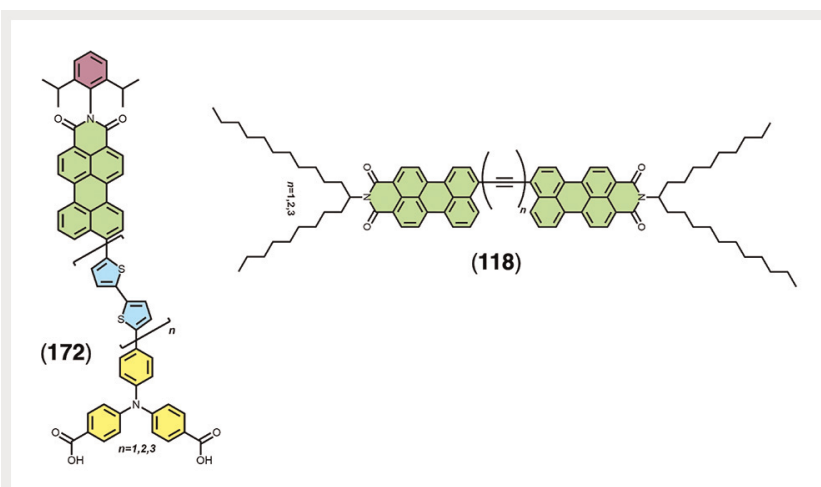

Figure 55 Spacer-based PMI sensitizers. ${ }^{82}$

most efficient sensitizer in the above series was the one with hexathiophene bridge recording a PCE value of $0.41 \%$.

Introduction of an alkyne spacer exhibits a good electrontransporting ability which has been exploited in three acetylene-bridged PMI dimers. PMI 118 in conjecture with another donor and acceptor pair has been used to fabricate ternary solar cells to achieve a PCE value of $9.77 \%$.

Thus, in a nutshell PMI has emerged as an obvious choice for organic photovoltaics owing to its versatility. However, unlike its sister analogue PDI, it has not been explored so passionately and hence provides a promising avenue to be delved into by the scientific community.

\section{$23 \mathrm{PMI}$ as a Fluorescent Reporter}

PMI dyes are promising candidates for sensing applications due to their high molar extinction coefficient, emission QY, and robust stability. However, there are only a few reports on the use of PMI for sensing as compared to the PDI dyes. The electron-deficient nature of PMI due to the imide group, large planer $\pi$-electron surface, and the donor-acceptor properties by the incorporation of a suitable electron-donating group have been utilized for sensing applications.

Our group has utilized the electron-deficient nature of PMI to develop a sensor for electron-rich aromatic amines. ${ }^{29}$ The PET from aromatic amine to PMI results in quenching of the fluorescence of PMI. We have synthesized a series of peri-functionalized PMI derivatives using Suzuki coupling reactions (vide supra) for sensing applications (see Figure 56). The electron transfer free energy of the PET process has been calculated and the negative free energy of the process favors the electron-transfer process. The peri-functionalization of PMI makes the reduction process much more facile compared to the unsubstituted PMI. The large negative free energy associated with the electron transfer process confirms the feasibility of the process.

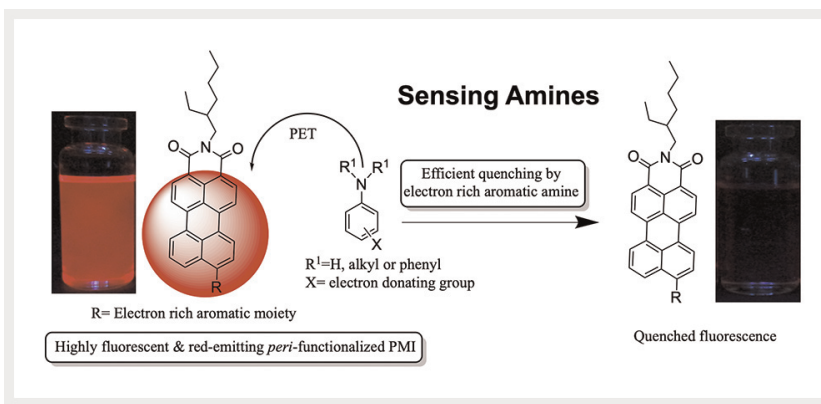

Figure 56 Sensing of electron-rich aromatic amine using peri-functionalized PMI derivatives via photo-induced electron transfer. Reprinted with permission from Ref. 29. Copyright 2017 Wiley-VCH.

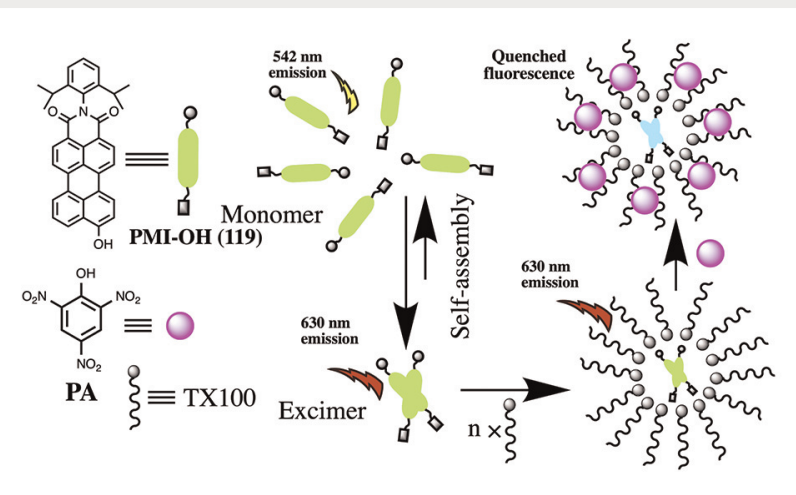

Figure 57 Fluorometric detection of picric acid using a hydroxylfunctionalized PMI derivative encapsulated into a micellar system.

Recently, Yu and co-workers have reported a micellar system for selective and sensitive detection of picric acid using a hydroxyl-functionalized PMI derivative (PMI-OH, 119). ${ }^{83}$ The authors have prepared a self-assembled micellar system using non-ionic surfactant TX-100 and hydrophobic PMI-OH (as shown in Figure 57). The incorporation of PMI-OH in the hydrophobic core of the micellar environment exhibits an intense excimeric emission. The electron transfers from the donor PMI-OH to the electron-deficient explosive acceptor results in a significant fluorescence quenching and ultimately a selective and sensitive detection of picric acid.

In the most recent report, our group has demonstrated a novel sensing concept on tuning donor-acceptor properties via the oxidation of a solid-state peri-annulated PMI derivative (shown in Figure 58)..$^{60}$ We reported a colorimetric and fluorogenic detection of harmful peroxide in organic solvents. The products obtained from the oxidation solid-state group results in a couple of new solid-state emissive fluorophores (vide supra).

Wilton-Ely and colleagues reported a series of new ruthenium(II) vinyl complexes by incorporating a peri-functionalized PMI unit (shown in the top-left part of Figure 59). 


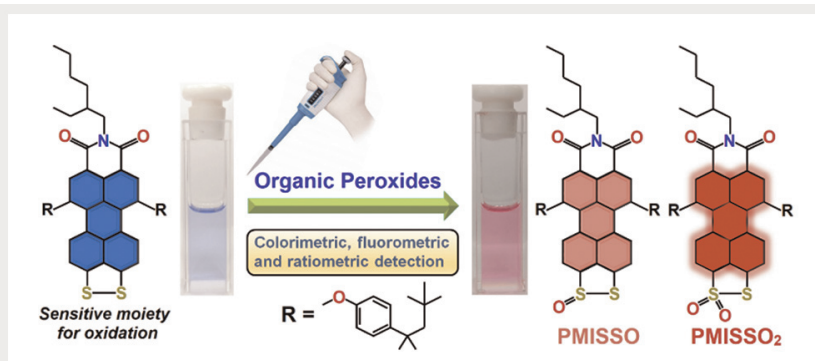

Figure 58 Colorimetric, fluorometric, and ratiometric detection of organic peroxide using solid-state peri-annulated PMI. Reprinted with permission from Ref. 60. Copyright 2021 Royal Society of Chemistry.

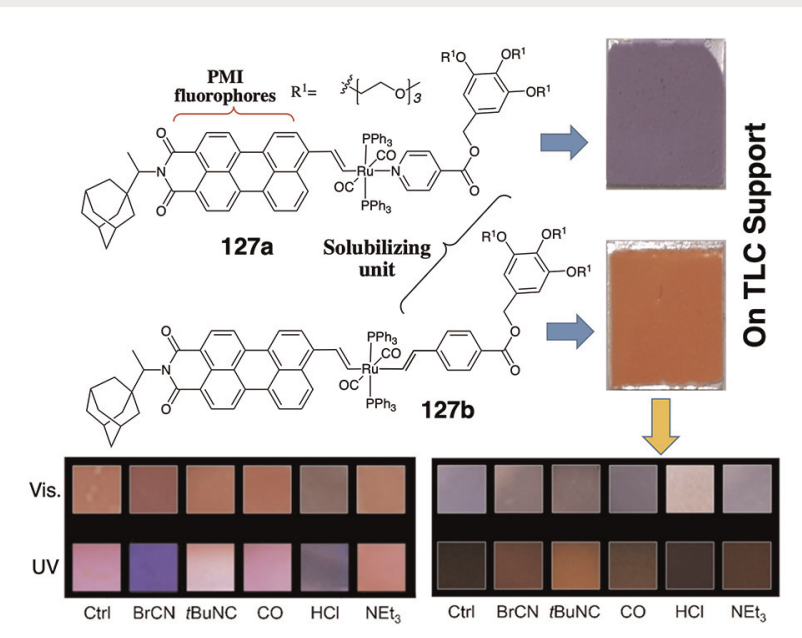

Figure 59 PMI-functionalized Ru(II) complexes for detection of toxic analytes on a silica support. The bottom panel shows the color of PMI-pyridyl (left) and PMI-vinyl (right) absorbed on silica support in presence of different analytes under visible and UV light. Reprinted with permission from Ref. 84. Copyright 2019 Wiley VCH.

The fluorogenic PMI moiety was functionalized with $\mathrm{Ru}(\mathrm{II})$ with the terminal vinyl or pyridyl groups $(\mathbf{1 2 7} \mathbf{a}, \mathbf{b})$ and the inherent low solubility of PMI in an aqueous environment was improved using three poly-PEGylated units. ${ }^{84}$ For detection of vapor and gases in the solid state, the compounds were absorbed on a thin layer of silica. The fluorescence modulation behavior was observed in solution as well as in solid silica matrix upon the reaction of the metal complexes with carbon monoxide, isonitrile or cyanide, hydrochloric acid, and amine vapor (see bottom part of Figure 59). These probes showed very low micromolar limits of detection for cyanide and tertiarybutylisonitrile in solution as well as for that immobilized on a silica matrix.

Very recently, Wang's group has developed a water-soluble PMI derivative using a triple-mannose group and modified the peri-position with 3-aminopyrrolidine for selective binding of double-stranded DNA. ${ }^{85}$ PMI-Man shows a signif-

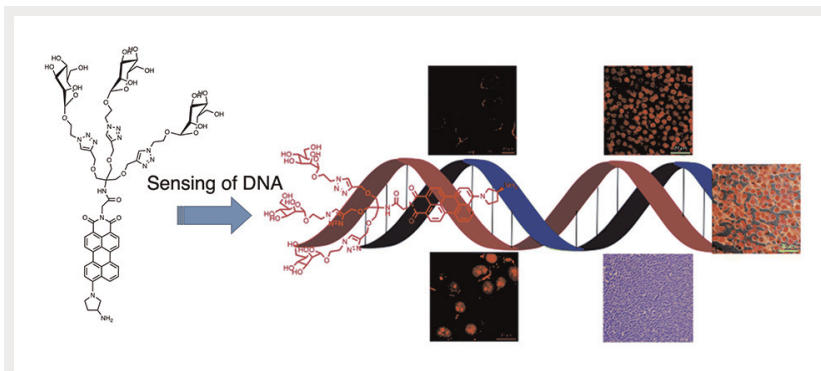

Figure 60 Structures of PMI-Man (126) and illustrations of double-DNA targeted live/dead cells and histological imaging using PMI-Man (126). Reprinted with permission from Ref. 85. Copyright 2021 Royal Society of Chemistry.

icant fluorescence enhancement upon DNA binding. This approach can be utilized for staining dead and live cells for potential application in the histological diagnosis of human tissue section (shown in Figure 60).

\subsection{Application of PMI Derivatives for Bio-Imaging}

Fluorescence-based techniques are non-invasive and have been extensively utilized for understanding the dynamical aspects of biological processes with high selectivity and sensitivity. Therefore, the development of new biocompatible fluorescence probes for cellular imaging with suitable functionality has become an important research avenue for understanding the cellular process. There are few important features of such bio-imaging probes which should be kept into consideration such as solubility in physiological conditions, low-energy background-free signal, high visible light absorption ability, strong emissive properties with large Stokes shift, and most importantly the photostability for long-term fluorescence imaging. In most cases, PMI derivatives have the aforementioned desirable properties as fluorescent probes except their workable solubility in an aqueous environment. Herein, we wish to provide an overview based on the recent reports on the application of PMI derivatives for in vitro and in vivo fluorescence imaging.

\subsection{Hydrophilic PMls for Bio-Imaging}

There are only quite a few number of water-soluble PDI derivatives that are known for their biological application and their utility in fluorescence imaging has been reviewed recently. ${ }^{20}$ However, water-soluble PMI is sparsely reported. In 2017, Zhu and co-workers designed and synthesized a novel type of PEGylated amphipathic and fluorescenceswitchable probe appended with DTE (PEG-PMI-DTE, 169, for structure see Figure 61, top part) for super-resolution fluorescence imaging of liposomal vesicles (multilamellar, 


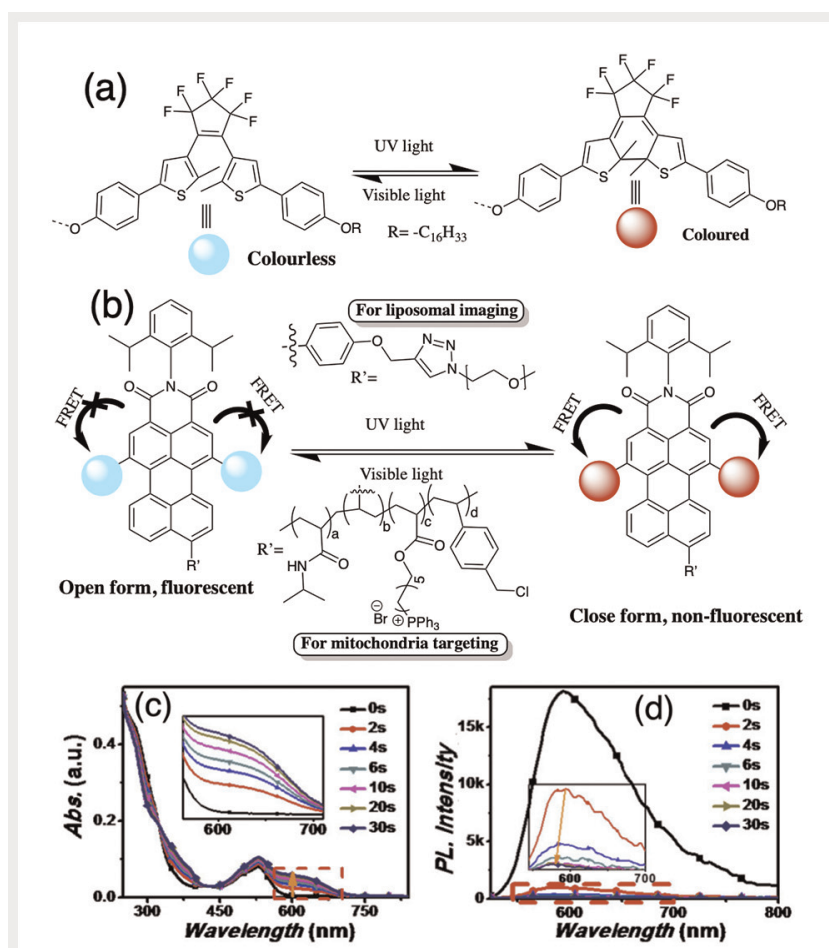

(e)

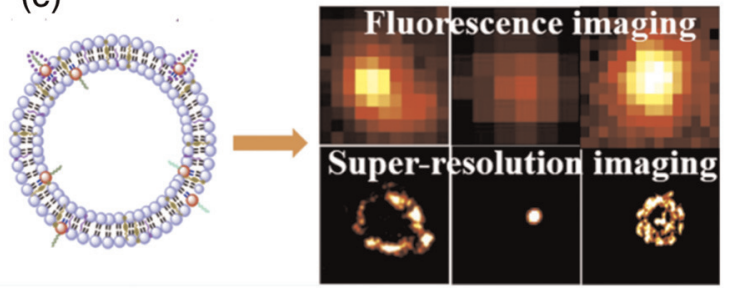

Figure 61 Application of photo-switchable PMI derivatives for superresolution imaging. (a, b) PMI was functionalized with photoswitchable dithienylethene (DTE) derivatives at the bay-position and judicially selected targeting group at the peri-position for liposomal vesicles and mitochondria. (c, d) There is a FRET from the PMI core to the closed form of DTE resulting in fast fluorescence quenching and no FRET with an open form. (e) This photoswitching is utilized for the super-resolution imaging. Reprinted with permission from Ref. 86. American Chemical Society.

large unilamellar, and small unilamellar vesicles). ${ }^{86}$ PEG-PMI-DTE exhibits excellent fatigue-resistant photochromic properties along with fluorescence switching upon UV-light and visible-light irradiation (Figure 61c,d). The fine nanostructures of liposomes with an optical resolution of $30 \mathrm{~nm}$ were obtained under a super-resolution microscope upon staining with PEG-PMI-DTE. ${ }^{86}$ The same group has reported mitochondria-targeting water-soluble fluorescence-switching probes based on the aforementioned construct using PMI-DTE. ${ }^{87}$ The PMI-DTE probe appended with a mitochondria-targeting triphenylphosphonium moiety (170) pos-

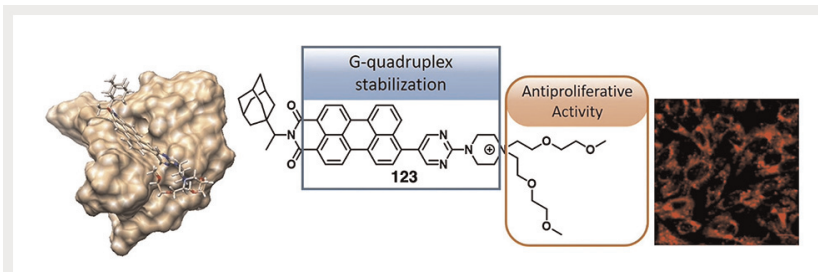

Figure 62 G-quadruplex stabilizing peri-functional PMI derivative with antiproliferative activity. Reprinted with permission from Ref. 88. Copyright 2021 Elsevier.

sesses high fluorescence-switching properties essential for super-resolution fluorescence imaging.

Busto and co-workers have synthesized a series of watersoluble PMI derivatives decorated at the peri-position with pyridine and pyrimidine moieties for G-quadruplex (G4) binding. ${ }^{88} \mathrm{G}$-quadruplex plays an important role in DNA replication, transcription, and genome stability, and is consequently associated with several diseases. PMI derivative 123 shows fluorescence enhancement upon G4 binding and this is related to thermal stabilization of G4. PMI 123 bears a permanent positive charge along with a PEG chain and results in an antiproliferative property. These molecules have high biocompatibility on cancer and non-cancerous cells and their potential for fluorescence imaging probe was assayed (Figure 62). The subcellular distribution of PMI 123 has been studied in human embryonic kidney cells (HEK293) by confocal microscopy. PMI 123 displays a red emission with a high degree of localization in mitochondria.

The development of dyes with NIR absorption is getting immense attention. In past decades, there were several small molecular and nano-objects developed in the traditional NIR-I window (700-900 nm) and have been investigated in both live animals and clinics. ${ }^{89}$ However, NIR-I is restricted to limited tissue penetration depth and photon scattering. Thus the NIR-II window (1000-1700 nm) with deeper tissue penetration and enhanced spatial resolution has much improved properties for background-free imaging. Rylene dyes have potential for achieving the NIR-II window with suitable modifications. ${ }^{53}$ Jana and co-workers have developed a PMI-based lysosome-specific NIR fluorescent probe (130) for fixed and live-cell imaging of cancer cells. ${ }^{90}$ The authors have used morpholine-appended PMI functionalized at the peri-position. The PMI derivative emits in the NIR range with the emission maxima around $700 \mathrm{~nm}$. Livecell co-localization experiments with a known organelle tracker showed that the PMI derivative selectively localizes in the lysosomal compartment of the cells (as shown schematically in Figure 63). The lysosomal dynamics were also monitored in live MCF7 cells. Finally, the applicability of the NIR-emitting PMI derivative to visualize the bio-distribution was assessed in a mouse model. 


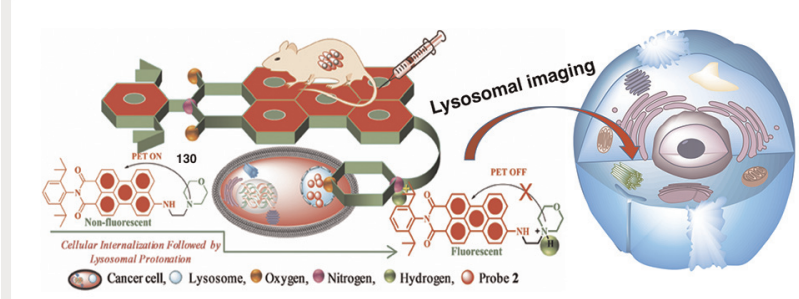

Figure 63 Water-soluble lysosome-targeting PMI derivative for lysosomal imaging in cells and in animals. Reprinted with permission from Ref. 90. Copyright 2019 Royal Society of Chemistry.

\subsection{Aggregated PMI as a NIR-Emissive Fluoroprobe for Bio-Imaging}

As discussed, PMI dyes are well known to have aggregation propensity which causes a remarkable change in their absorption and emission properties. There are few reports on the application of such modulated optical properties of aggregated PMI for bio-imaging.

Zhao and colleagues reported aggregation-induced excimer NIR emission $(\sim 700 \mathrm{~nm})$ from a suitably substituted PMI derivative in an aqueous medium. ${ }^{91}$ Aggregation of the PMI derivatives forms organic NPs (PMI-NPs). They used the re-precipitation technique for synthesizing PMI-NPs, their size and morphology were tuned by a self-assembling approach. The self-assembly of PMI-NPs to yield giant NPs (colloidosomes) has been arrested using a non-ionic surfactant pluronic F127 (EM-PMI@Plu127). Interestingly, the greenemissive PMI (emission maxima $\sim 550 \mathrm{~nm}$ ) can be transformed into a NIR-emitting fluorophore (emission maxima $\sim 700 \mathrm{~nm}$ ) in an aqueous solution by allowing it to form the excimer in the aggregated state (see Figure 64a). A controlled re-precipitation of PMI with F127 further gave a green-emitting particle with a size distribution of 5-15 nm (MM-PMI@Plu127). Furthermore, the nanosystem developed by Zhao's group by using controlled self-assembly of PMI finds applications in live-cell fluorescence imaging as shown in Figure 64b.

In 2018, we have also developed a NIR-emissive PMIdoped polymer NP for selective targeting of folate receptors for differentiating cancer cells from normal cells. ${ }^{31}$ An amphiphilic co-polymer polystyrene-PEG caused a nanospaceinduced aggregation of PMI derivatives resulting in NIR emission. Later, the polymer was functionalized with folic acid to selectively target folate-receptor over-expressing cancer cells (as shown in Figure 65a-c).

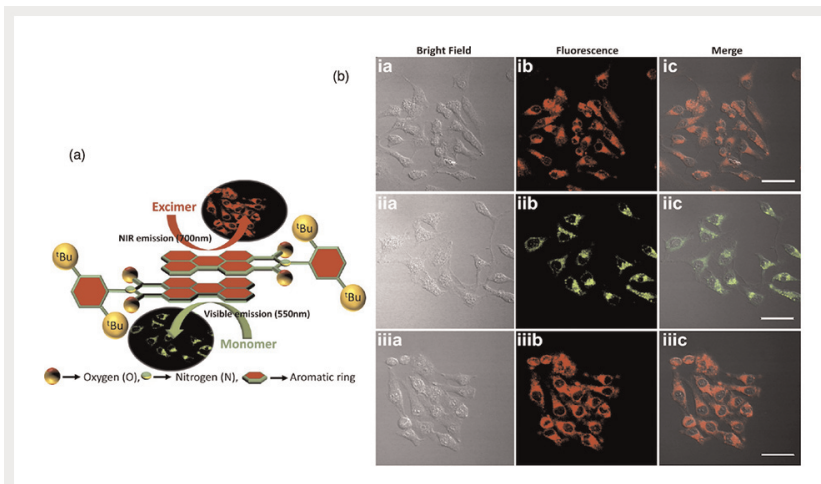

Figure 64 (a) Schematic representation of aggregation-induced excimeric NIR emission and preservation of monomeric emission from PMI. (b) Live-cell CLSM imaging with HeLa cells (a) bright-field, (b) fluorescence, and (c) merged images incubated with (i) PMI-NPs in water, (ii) green-emitting MM-PMI@Plu127, and (iii) NIR-emitting EM-PMI@Plu127 (scale bar $=50 \mu \mathrm{m}$ ). Emission was recorded using a $550-\mathrm{nm}$ channel for the green emission and a 700-nm channel for the NIR emission. Reprinted with permission from Ref. 91. Copyright 2017 American Chemical Society.

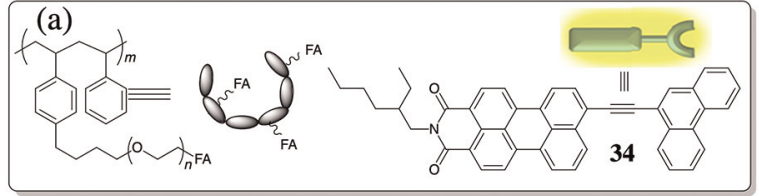

(b)
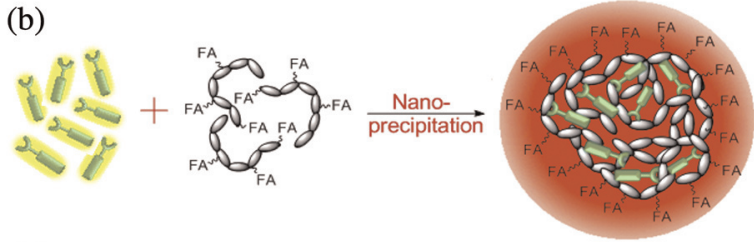

(c)
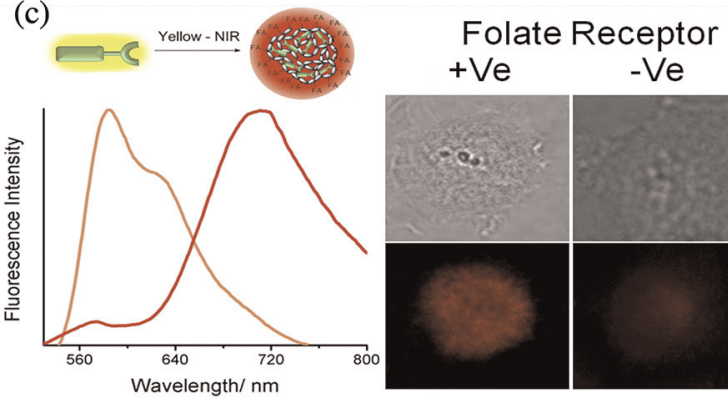

$+\mathrm{Ve}$

$-\mathrm{Ve}$

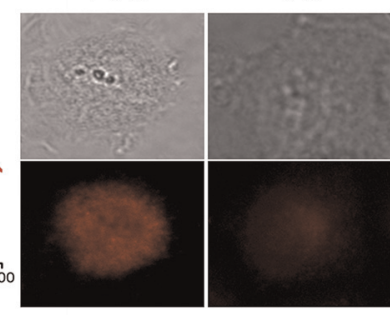

Figure 65 NIR emission coming from the aggregation properties of PMI-appended phenanthrene (34) due to the confinement effect originated from the self-assembled amphiphilic polystyrene-PEG polymer functionalized with folic acid (a, b) for selective imaging of folate receptor over-expressing cancer cells using NIR-emissive polymer nanoparticles (c). Reprinted with permission from Ref. 31. Copyright 2017 Royal Society of Chemistry. 


\section{Photocatalytic Hydrogen Generation Using PMI}

Stupp and co-workers have utilized the supramolecular chromophoric hydrogel materials in combination with a nickel catalyst for light-driven $\mathrm{H}_{2}$ production (as shown in Figure 66a). ${ }^{34}$ They have synthesized a visible-light absorbing PMI chromophore amphiphile (CA) by covalently functionalizing a five-carbon carboxylic acid (116a-d, for structure see Figure 66b). Later, the authors have explored CA gels as light-absorbing units in a supramolecular catalytic system using a water-soluble proton reduction catalyst [ $\mathrm{Ni}$ $\left(\mathrm{P}_{2} \mathrm{PhN}_{2} \mathrm{Ph}\right)_{2}\left(\mathrm{BF}_{4}\right)\left(\mathrm{NiP}_{2} \mathrm{~N}_{2}\right)$ or thiomolybdate cluster, $\left(\mathrm{NH}_{4}\right)_{2}$ $\left[\mathrm{Mo}_{3} \mathrm{~S}_{13}\right]$ with ascorbic acid as a proton source and sacrificial electron donor. The $\mathrm{H}_{2}$ evolution experiments show that the CA-PDDA gel was capable of photosensitizing a nickel catalyst for $\mathrm{H}_{2}$ evolution and no $\mathrm{H}_{2}$ evolution was observed when either of chromophore, catalyst, sacrificial reagent, or light was omitted from the system.

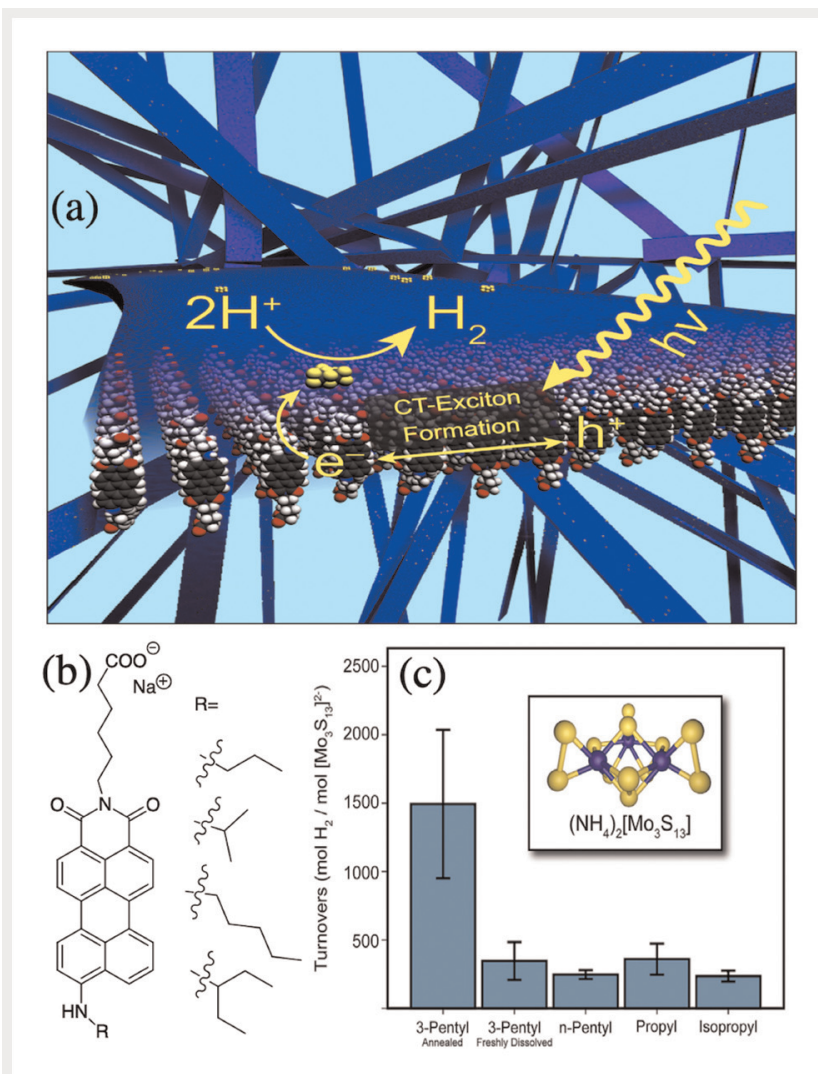

Figure 66 (a) Nanoscale ribbons from the self-assembly of PMI derivatives are used to collect light and transfer the energy to a catalyst for the generation of hydrogen gas, (b) representative examples of PMI derivatives used for such photocatalytic applications, and (c) plot of TON with different derivatives. Reprinted with permission from Ref. 34 . Copyright 2018 Elsevier.
Reisner and co-workers reported the synthesis of five different peri-substituted PMI derivatives with different anchoring functionality, utilized for DSSC and photocatalytic applications in an aqueous medium. ${ }^{11 \mathrm{~b}}$ Different anchoring groups were introduced to modulate the binding strength and proximity, which in turn controls the electronic communications between the PMI dyes and $\mathrm{TiO}_{2} \mathrm{NPs}$. The effect of the anchoring group of five PMI dyes was systematically studied side-by-side in DSC and dye-sensitized photocatalysis systems, at basic, neutral, and acidic $\mathrm{pH}$, in combination with $\mathrm{I}^{3-} / \mathrm{I}^{-}$, ascorbic acid or triethanolamine as mediators or sacrificial electron donors. They have reported production of $54 \mu \mathrm{mol}$ of $\mathrm{H}_{2}$ with $\mathbf{P M I}-\mathbf{C O}_{2} \mathbf{H}$ derivative giving an initial turnover frequency (TOF) of $344 \mathrm{~h}^{-1}$ and the highest turnover number (TON) of approximately 6460 after one day of UV-filtered simulated solar light irradiation.

The organization of synthetic chromophores into supramolecular assemblies presents an appealing approach to the construction of solar-to-fuel conversion materials. Inspiration arises from natural synthetic machinery, where chromophores are precisely organized into arrays that transfer energy over long distances to fuel-producing catalysts. ${ }^{92}$ To mimic such precise organization, Stupp and his co-workers synthesized a series of PMIs with varying dipole moments in which an electron-donating amine, an electron-neutral methyl, or an electron-withdrawing nitrile were attached to the 9-position of PMI's aromatic core. Substitutions at this position influence the electronic properties such as bandgap, HOMO-LUMO levels, and dipole strength more than at other positions of PMI.

During the photocatalytic study for hydrogen generation, they found that electronic properties (dipole strength) of synthesized PMIs have a profound effect on both the chromophore assembly (CAs) and morphology and ultimately upon its catalytic efficiency. ${ }^{93}$ Interestingly, they found that the intermediate dipole moment shown by CAs made of $\mathbf{C H}_{\mathbf{3}}$-PMI yields greater hydrogen generation than the other synthesized PMIs. Thus dipole-dipole interactions represent an important criterion for understanding how tailoring chemical structure modifies the molecular packing and controls light-harvesting properties of self-assembled architectures, a unique aspect of supramolecular materials. On the same concept, the same group reported CAs based on PMI derivatives bearing alkyl tails attached via an amine group at the 9-positions. ${ }^{34}$ The large molecular dipole moment imparted by the amine group at the peri-position and sterically demanding alkyl groups determine the internal order, morphology, and electronic structure of the CAs. The crystalline assemblies that support the coupling of transition dipole moment coupling and charge-transfer excitons are useful for photocatalytic hydrogen production. For the photocatalytic hydrogen production, CAs were investigated by forming hydrogels with the addition of polydiallyl dimethyl ammonium chloride. Next, the hydrogels were illuminated in 
the presence of a proton reduction catalyst (thiomolybdate cluster, $\left.\left(\mathrm{NH}_{4}\right)_{2}\left[\mathrm{Mo}_{3} \mathrm{~S}_{13}\right]\right)$ and ascorbic acid as a source of protons and a sacrificial electron donor, respectively. The authors have achieved a TON of $\sim 2000 \mathrm{molH}_{2} / \mathrm{mol}$ with the annealed 3-pentyl derivative of PMI (as shown in Figure 66c).

Stupp and his team demonstrated the impact of charge switching using the $\mathrm{pH}$ of the medium on supramolecular assemblies of PMI. Such pH-switchable assemblies were utilized as a PS in photocatalytic production of hydrogen with $\left(\mathrm{NH}_{4}\right)_{2}\left[\mathrm{Mo}_{3} \mathrm{~S}_{13}\right]$ as a catalyst at $\mathrm{pH} 4.0 .^{68}$ The bandgap of PMI assemblies is sensitive to the local $\mathrm{pH}$ of the medium and has been utilized for photocatalytic applications. Hydrogen was generated with a TOF of 125 after $18 \mathrm{~h}$. However, at higher $\mathrm{pH}$ ( $\mathrm{pH} 9-10)$, in conjunction with a water-soluble iron-porphyrin catalyst, the assemblies can be applied to reduce proton to hydrogen and carbon dioxide to carbon monoxide with a TOF value of 30 .

Of late, Peneva's group has discovered a simple fourfold substitution strategy on PMI by which they have synthesized a number of $-\mathrm{XPh}$ group appended PMI derivatives $(\mathbf{1 6 5}, \mathbf{1 6 6}, \mathbf{1 6 7}$, and 168), given in Figure 67a. The newly discovered compounds were characterized by several spectroscopic and electrochemical techniques. ${ }^{28}$ But, the most enthralling story lies in their hydrogen evaluation catalytic activity. They have used the tetra-substituted PMI as PS in combination with $\left(\mathrm{NH}_{4}\right)_{2}\left[\mathrm{Mo}_{3} \mathrm{~S}_{13}\right] \cdot 2 \mathrm{H}_{2} \mathrm{O}$ as the hydrogen evaluation catalyst. The mechanism behind this light-driven catalysis underlies firstly the photoexcitation of PMI as PS, followed by electron transfer between the long-lived excited triplet state of PS and the $\left[\mathrm{Mo}_{3} \mathrm{~S}_{13}\right]^{2-}$ catalyst and reduction of PS in the presence of the sacrificial donor as depicted in Figure 67b. It was observed that, among three derivatives, compound 167 was the most efficient performer in lightdriven hydrogen evaluation catalysis with a TON of $10^{4}$ after 3 days of photoirradiation, whereas compound 165 was inactive in this process, as shown in Figure 67c. The inactivity of compound $\mathbf{1 6 5}$ is presumably ascribed to a very shortlived excited state which mostly favours its radiative pathway. On the search of novel organic chromophores in photo-

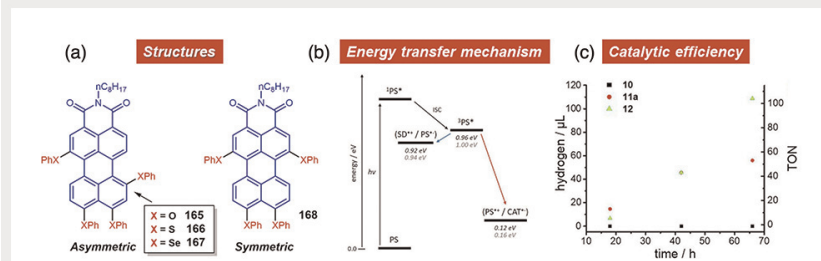

Figure 67 (a) Chemical structures of tetra-functionalized PMI derivatives. (b) Schematic diagram of the energy transfer process during photocatalysis. (c) Photocatalytic hydrogen production of $\left[\mathrm{Mo}_{3} \mathrm{~S}_{13}\right]^{2-}$ in the presence of compounds 165,166 , and 167 . Reprinted with permission from Ref. 28. Copyright 2021 Wiley VCH. catalysis, this idea has opened a new window on further tetra-functionalization of PMI by new substituents to increase the catalytic efficiency.

\section{PMI-Based Organocatalysis}

In 2019, Brouwer and his co-workers have synthesized cinchona alkaloid-conjugated PMI (122a, b) derivatives for organocatalytic applications. ${ }^{51}$ A suitable PMI-BnCPD derivative (122b) was used to assess the organocatalytic activity via a turn-on fluorescence response originated from the Michael addition reaction between a BODIPY-maleimide conjugate and benzyl mercaptan (as shown in Figure 68).

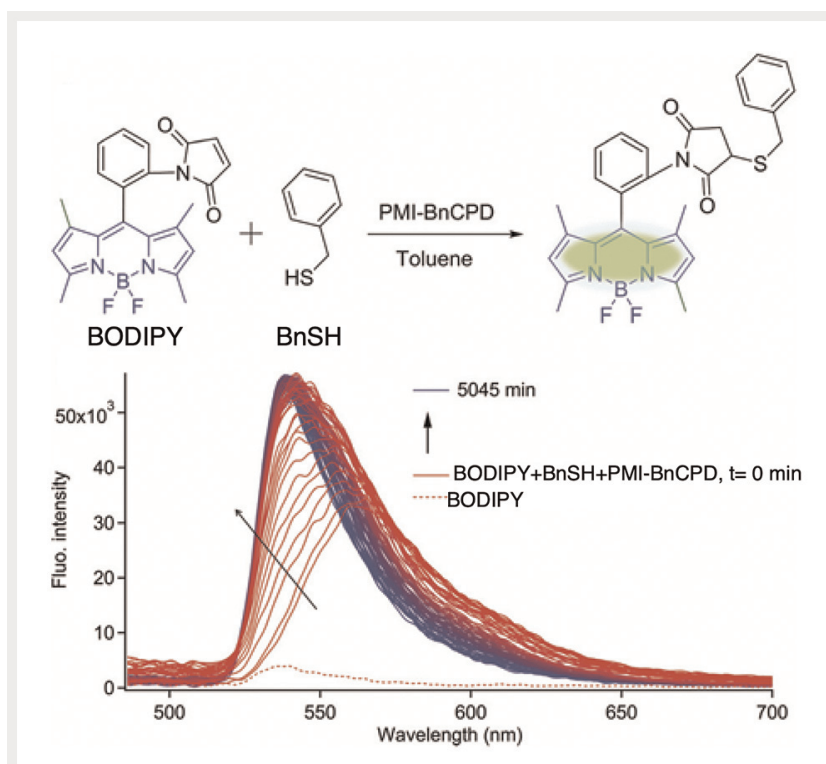

Figure 68 Application of PMI-BnCPD (122b) for organocatalysis. Michael addition between the BODIPY-maleimide conjugate and benzyl mercaptan results a fluorescent product. Reprinted with permission from Ref. 51. Copyright 2019 Royal Society of Chemistry.

\section{PMI Derivatives for Single-Molecule Spectroscopy}

SMS has increasingly become popular due to its applications in physics, chemistry, and biological sciences. SMS has provided new insights into electronic interaction when various emitters such as quantum dots, light-harvesting complex, conjugated polymer, and multi-chromophoric molecules were investigated under different conditions. In general, the bulk photophysical properties are very different from the single-molecule behavior. The most interesting and intensively studied single-molecule property is the reversible and stochastic fluorescence intermittency property switch- 
ing in the fluorescence intensity often called as fluorescence blinking.

Müllen and co-workers in collaboration with Basche's group have expensively investigated the blinking properties of PMI derivatives (Figure 69a) in a thin polycyclo-olefin polymer film spin-coated on a glass cover slide..$^{94}$ They have reported the comparison of the bulk spectroscopic and the single-molecule properties of dimeric PMI derivatives (133) to understand the electronic coupling of monomers. The fluorescence intensity trajectories at the single-molecule level show one-step and two-step bleaching behaviour (Figure $69 \mathrm{~b}, \mathrm{c}$ ). In this experiment, $32 \%$ of molecules show twostep behavior and for others, time traces were more complex with multiple reversible dark-state transitions, might be due to triplet excursions. The simultaneous recording of emission spectrum in different time-points of the trajectories indicated that the dipole-dipole interaction is important for single-molecule behavior and provided a signature of monomeric coupling.

Later, the same group has investigated the fluorescence intermittency behavior of a peri-functionalized PMI derivative containing a small dendritic arm in different polymer matrices and different environmental conditions (Figure 69d, e) ${ }^{44}$ The duration of the dark state follows a power-law dependence, which was due to the formation of radical cation/anion due to charge tunnelling associated with widely distributed charge-trapping states.

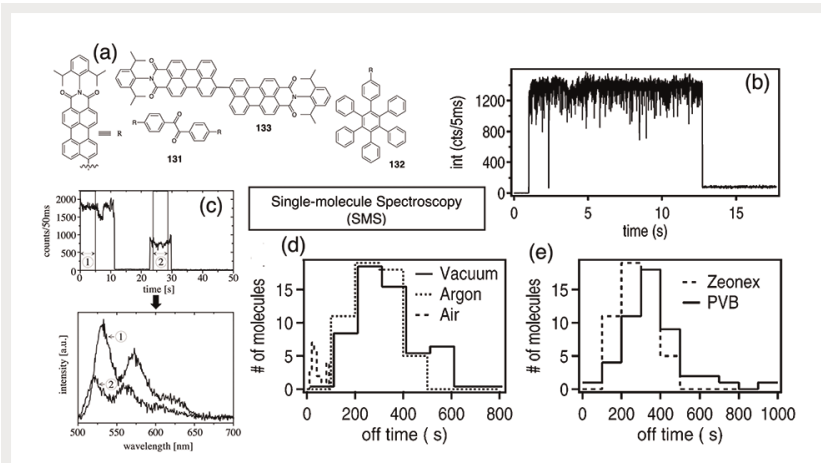

Figure 69 Single-molecule investigation of PMI derivatives. (a) Structures of PMI used for single-molecule spectroscopy, (b) a representative time trajectory, (c) two-step bleaching behavior and the corresponding emission spectrum at the selected time point, and (d) effect of medium and (e) the host materials. Figure 69b and c were reprinted with permission from Ref. 94. Copyright 2002 Elsevier. Figure 69d and e were reprinted with permission from Ref. 44. Copyright 2004 American Chemical Society.

\section{Host-Guest Chemistry with PMI}

Recently, PMI derivatives are finding applications in hostguest complexation with cucurbit[8]uril (CB8). Hunter and co-workers reported supramolecular host-guest complexation of a water-soluble PMI derivative and CB8. The authors have observed a strong fluorescence of 19 -fold enhancement due to de-aggregation and complexation of PMI by $\mathbf{C B 8} .^{95}$ Finally, the highly emissive PMI•CB8 complex has been used to study the complexation of strong CB8 binders like adamantylamine, methyl viologen, amino acids, and tripeptides (see Figure 70a).

Later in 2018, Jayawickramarajah and his team reported the complexation of three positively charged PMI derivatives with CB8 to understand their structural effect on host-guest binding. ${ }^{96}$ The authors have functionalized the PMI at the imide position by modifying the spacer between the imide position and phenyl group for binding with CB8. The binding properties of PMI derivatives and CB8 were assessed using various spectroscopic techniques. PMI derivatives show strong micromolar affinity and the complex is highly emissive. Later, the authors have used a highly emissive CB8・PMI complex for the displacement assay for the detection of a wide range of CB8-binding guests (as shown in Figure 70b).

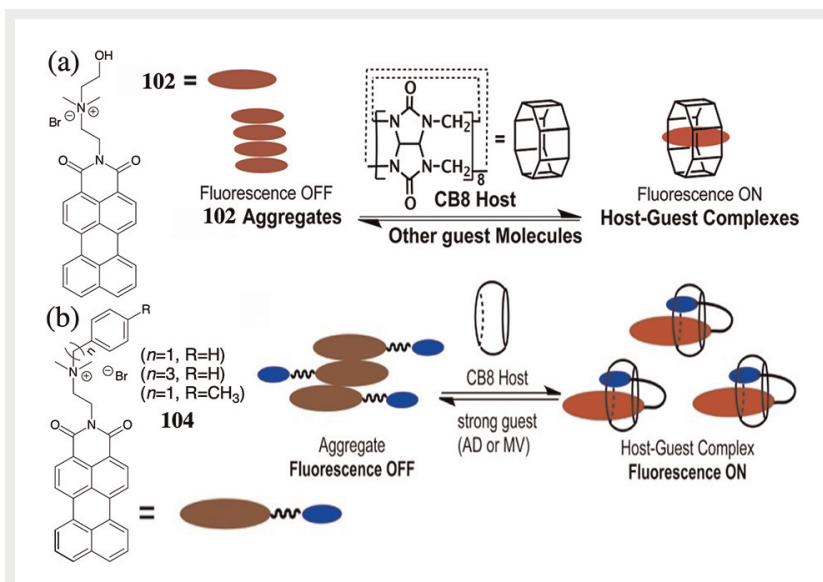

Figure 70 Positively charged imide-functionalized PMI derivatives (left) were used for complexation with CB8. CB8 complexation causes a turnon emission due to the de-aggregation of PMI. The emissive complexes were utilized to develop a displacement assay for understanding the binding of other guest molecules (shows in right). Figure 70a was reprinted with permission from Ref. 95. Copyright 2016 Royal Society of Chemistry. Figure 70b was reprinted with permission from Ref. 96. Copyright 2016 Wiley VCH. 


\section{Conclusions and Outlook}

In this contribution, we have attempted to accentuate the tremendous progression that has swept across the PMI realm during the last two decades. PMI has already emerged as an exemplar in functional organic materials on account of not only its distinctive photophysical, electrochemical and self-assembling properties, but also due to its magnificent applications in the domain of organic optoelectronics, photocatalysis, fluorescence sensing, and bio-imaging as discussed in this review. Although with the pioneering contribution of several eminent groups, the research voyage of this profoundly sought-after dye is progressing smoothly, yet much of its potentiality still remains to be explored. From the synthetic viewpoint, engineering of the most reactive and tunable peri-position either by peri-functionalization or by ring fusion offers a prospective research terrain for begetting new donor-acceptor architectures relevant to organic electronics. Additionally, tetra-substitution in the PMI core, invented by our group, endows a great opportunity to remarkably tailor the spectro-electrochemical properties of PMI by judicious choice of substituents. PDI-based ambientstable radical anions are abundant, in contrast, PMI-based ones are yet to come forth. Thus, design and synthesis of PMI-based ambient stable radical anion would illumine new avenues in molecular magnetism, catalysis, organic spintronics and electronics. In comparison to the phenomenal application of PMI in organic optoelectronics, its biological applications reported hitherto are at a preliminary stage mostly due to the cumbersome preparation of watersoluble PMI derivatives. Synthesis of water-soluble PMI derivatives pertinent to bio-imaging and bio-labelling calls for cognizance from the scientific community. Ultrafast transient processes like SF, SBCT and other excited-state dynamics using novel PMI scaffolds afford intriguing research domains to the physical chemists. Commensurating with a large hydrophobic surface that exhibits self-assembling propensity, PMI indeed furnishes a wide scope of delving into its self-assembling property for modulating its photophysical properties for utilisation in organic photovoltaics and several excited-state phenomena.

\section{Funding Information}

We thank the Council of Scientific \& Industrial Research (CSIR), India, for the funding (grant number: 02(0134)/17/ EMR-II) of our perylene-based work.

\section{Acknowledgment}

We would like to thank Dr. Dhananjaya Sahoo and Dr. Vikas Sharma for developing the PMI work in our group at IISER Bhopal. We acknowledge the anonymous reviewers for their insightful comments and suggestions to improve the state of this manuscript. We wish to acknowledge the contribution of all researchers working on perylene for their passion and hard work for the development of beautiful perylene chemistry. R. R. thanks IISERB for his doctoral fellowship. A. K. and O.C. acknowledge doctoral fellowship support from UGC and DST INSPIRE, MHRD, India, respectively.

\section{Conflict of Interest}

The authors declare no conflict of interest.

\section{References}

(1) (a) Wächtler, M.; González, L.; Dietzek, B.; Turchanin, A.; Roth, C., Phys. Chem. Chem. Phys. 2019, 21, 8988; (b) Ostroverkhova, O., Chem. Rev. 2016, 116, 13279.

(2) (a) Würthner, F., Chem. Commun. 2004, 1564; (b) Würthner, F.; Saha-Möller, C. R.; Fimmel, B.; Ogi, S.; Leowanawat, P.; Schmidt, D., Chem. Rev. 2016, 116, 962.

(3) Chen, L.; Li, C.; Müllen, K., J. Mater. Chem. C 2014, 2, 1938.

(4) (a) Weil, T.; Vosch, T.; Hofkens, J.; Peneva, K.; Müllen, K., Angew. Chem. Int. Ed. 2010, 49, 9068; (b) Zhan, X.; Facchetti, A.; Barlow, S.; Marks, T. J.; Ratner, M. A.; Wasielewski, M. R.; Marder, S. R., Adv. Mater. 2011, 23, 268; (c) Yan, C.; Barlow, S.; Wang, Z.; Yan, H.; Jen, A. K. Y.; Marder, S. R.; Zhan, X., Nat. Rev. Mater. 2018, 3, 18003; (d) Yuan, Z.; Lee, S.-L.; Chen, L.; Li, C.; Mali, K. S.; De Feyter, S.; Müllen, K., Chem. Eur. J. 2013, 19, 11842; (e) Liang, N.; Sun, K.; Feng, J.; Chen, Y.; Meng, D.; Jiang, W.; Li, Y.; Hou, J.; Wang, Z., J. Mater. Chem. A, 2018, 6, 18808.

(5) (a) Wonneberger, H.; Ma, C.-Q.; Gatys, M. A.; Li, C.; Bäuerle, P.; Müllen, K., J. Phys. Chem. B 2010, 114, 14343; (b) Andreas, H.; Klaus, M., Chem. Lett. 2006, 35, 978; (c) Gsänger, M.; Bialas, D.; Huang, L.; Stolte, M.; Würthner, F., Adv. Mater. 2016, 28, 3615.

(6) (a) Biswas, S.; Sharma, V.; Kumar, P.; Koner, A. L., Sens. Actuators, B 2018, 260, 460; (b) Biswas, S.; Upadhyay, N.; Kar, D.; Datta, S.; Koner, A. L., Nitric Oxide 2019, 92, 34; (c) Kumar, P.; Biswas, S.; Koner, A. L., New J. Chem. 2020, 44, 10771; (d) Biswas, S.; Dutta, T.; Silswal, A.; Bhowal, R.; Chopra, D.; Koner, A. L., Chem. Sci. 2021, 12, 9630.

(7) (a) Zhang, F.; Ma, Y.; Chi, Y.; Yu, H.; Li, Y.; Jiang, T.; Wei, X.; Shi, J., Sci. Rep. 2018, 8, 8208; (b) Türkmen, G.; Erten-Ela, S.; Icli, S., Dyes Pigm. 2009, 83, 297; (c) Zhang, Y.; Chen, L.; Zhang, K.; Wang, H.; Xiao, Y., Chem. Eur. J. 2014, 20, 10170; (d) Avlasevich, Y.; Li, C.; Müllen, K., J. Mater. Chem. 2010, 20, 3814.

(8) Zhao, X.; Xiong, Y.; Ma, J.; Yuan, Z., J. Phys. Chem. A 2016, 120, 7554.

(9) Kardos, M., DE Patent 276357, 1913.

(10) (a) Langhals, H.; Obermeier, A.; Floredo, Y.; Zanelli, A.; Flamigni, L., Chem. Eur. J. 2009, 15, 12733; (b) Ventura, B.; Langhals, H.; Böck, B.; Flamigni, L., Chem. Commun. 2012, 48, 4226; (c) Kölle, P.; Pugliesi, I.; Langhals, H.; Wilcken, R.; Esterbauer, A. J.; de Viv- 
ie-Riedle, R.; Riedle, E., Phys. Chem. Chem. Phys. 2015, 17, 25061; (d) Kirmaier, C.; Song, H. E.; Yang, E.; Schwartz, J. K.; Hindin, E.; Diers, J. R.; Loewe, R. S.; Tomizaki, K. Y.; Chevalier, F.; Ramos, L.; Birge, R. R.; Lindsey, J. S.; Bocian, D. F.; Holten, D., J. Phys. Chem. B 2010, 114, 14249; (e) Yang, E.; Wang, J. Q.; Diers, J. R.; Niedzwiedzki, D. M.; Kirmaier, C.; Bocian, D. F.; Lindsey, J. S.; Holten, D., J. Phys. Chem. B 2014, 118, 1630; (f) Hu, G. F.; Liu, R.; Alexy, E. J.; Mandal, A. K.; Bocian, D. F.; Holten, D.; Lindsey, J. S., New J. Chem. 2016, 40, 8032; (g) Kamm, V.; Battagliarin, G.; Howard, I. A.; Pisula, W.; Mavrinskiy, A.; Li, C.; Müllen, K.; Laquai, F., Adv. Energy Mater. 2011, 1, 297; (h) Dössel, L. F.; Kamm, V.; Howard, I. A.; Laquai, F.; Pisula, W.; Feng, X.; Li, C.; Takase, M.; Kudernac, T.; De Feyter, S.; Müllen, K., J. Am. Chem. Soc. 2012, 134, 5876; (i) Stappert, S.; Li, C.; Müllen, K.; Basché, T., Chem. Mater. 2016, 28 , 906; (j) Li, M.; Zajaczkowski, W.; Velpula, G.; Jänsch, D.; Graf, R.; Marszalek, T.; Parekh, S. H.; Zagranyarski, Y.; Mali, K.; Wagner, M.; De Feyter, S.; Li, C.; Müllen, K.; Pisula, W., Chem. Sci. 2020, 11, 4960; (k) Lin, M.-J.; Fimmel, B.; Radacki, K.; Würthner, F., Angew. Chem. Int. Ed. 2011, 50, 10847; (1) Troeger, A.; Ledendecker, M.; Margraf, J. T.; Sgobba, V.; Guldi, D. M.; Vieweg, B. F.; Spiecker, E.; Suraru, S.-L.; Würthner, F., Adv. Energy Mater. 2012, 2, 536; (m) Son, M.; Park, K. H.; Shao, C.; Würthner, F.; Kim, D., J. Phys. Chem. Lett. 2014, 5, 3601; (n) Herbst, S.; Soberats, B.; Leowanawat, P.; Stolte, M.; Lehmann, M.; Würthner, F., Nat. Commun. 2018, 9, 2646; (o) Schmidt, D.; Stolte, M.; Süß, J.; Liess, A.; Stepanenko, V.; Würthner, F., Angew. Chem. Int. Ed. 2019, 58, 13385; (p) Hartnett, P. E.; Timalsina, A.; Matte, H. S. S. R.; Zhou, N.; Guo, X.; Zhao, W.; Facchetti, A.; Chang, R. P. H.; Hersam, M. C.; Wasielewski, M. R.; Marks, T. J., J. Am. Chem. Soc. 2014, 136, 16345; (q) Hartnett, P. E.; Dyar, S. M.; Margulies, E. A.; Shoer, L. E.; Cook, A. W.; Eaton, S. W.; Marks, T. J.; Wasielewski, M. R., Chem. Sci. 2015, 6, 402; (r) Lindquist, R. J.; Phelan, B. T.; Reynal, A.; Margulies, E. A.; Shoer, L. E.; Durrant, J. R.; Wasielewski, M. R., J. Mater. Chem. A 2016, 4, 2880; (s) Hartnett, P. E.; Mauck, C. M.; Harris, M. A.; Young, R. M.; Wu, Y.-L.; Marks, T. J.; Wasielewski, M. R., J. Am. Chem. Soc. 2017, 139, 749.

(11) (a) Royakkers, J.; Minotto, A.; Congrave, D. G.; Zeng, W.; Patel, A.; Bond, A. D.; Bučar, D.-K.; Cacialli, F.; Bronstein, H., J. Org. Chem. 2020, 85, 207; (b) Warnan, J.; Willkomm, J.; Farre, Y.; Pellegrin, Y.; Boujtita, M.; Odobel, F.; Reisner, E., Chem. Sci. 2019, 10, 2758.

(12) (a) Nowak-Król, A.; Würthner, F., Org. Chem. Front. 2019, 6, 1272; (b) Ji, C.; Cheng, W.; Yuan, Q.; Müllen, K.; Yin, M., Acc. Chem. Res. 2019, 52, 2266.

(13) Schlosser, F.; Moos, M.; Lambert, C.; Würthner, F., Adv. Mater. 2013, 25, 410.

(14) (a) Hecht, M.; Würthner, F., Acc. Chem. Res. 2021, 54, 642; (b) Chen, S.; Slattum, P.; Wang, C.; Zang, L., Chem. Rev. 2015, 115 , 11967.

(15) Oleson, A.; Zhu, T.; Dunn, I. S.; Bialas, D.; Bai, Y.; Zhang, W.; Dai, M.; Reichman, D. R.; Tempelaar, R.; Huang, L.; Spano, F. C., J. Phys. Chem. C 2019, 123, 20567.

(16) (a) Zhou, J.; Zhang, W.; Jiang, X.-F.; Wang, C.; Zhou, X.; Xu, B.; Liu, L.; Xie, Z.; Ma, Y., J. Phys. Chem. Lett. 2018, 9, 596; (b) Zong, L.; Zhang, H.; Li, Y.; Gong, Y.; Li, D.; Wang, J.; Wang, Z.; Xie, Y.; Han, M.; Peng, Q.; Li, X.; Dong, J.; Qian, J.; Li, Q.; Li, Z., ACS Nano 2018, 12,9532

(17) (a) Wu, Y.; Young, R. M.; Frasconi, M.; Schneebeli, S. T.; Spenst, P.; Gardner, D. M.; Brown, K. E.; Würthner, F.; Stoddart, J. F.; Wasielewski, M. R., J. Am. Chem. Soc. 2015, 137, 13236; (b) Conrad-Burton, F. S.; Liu, T.; Geyer, F.; Costantini, R.; Schlaus, A. P.; Spencer, M. S.; Wang, J.; Sánchez, R. H.; Zhang, B.; Xu, Q.; Steigerwald, M.
L.; Xiao, S.; Li, H.; Nuckolls, C. P.; Zhu, X., J. Am. Chem. Soc. 2019 , $141,13143$.

(18) Sharma, V.; Koenig, J. D. B.; Welch, G. C., J. Mater. Chem. A 2021, 9, 6775.

(19) (a) Kumar, Y.; Kumar, S.; Bansal, D.; Mukhopadhyay, P., Org. Lett. 2019, 21, 2185; (b) Sharma, V.; Puthumana, U.; Karak, P.; Koner, A. L., J. Org. Chem. 2018, 83, 11458.

(20) Sun, M.; Müllen, K.; Yin, M., Chem. Soc. Rev. 2016, 45, 1513.

(21) Edvinsson, T.; Li, C.; Pschirer, N.; Schöneboom, J.; Eickemeyer, F.; Sens, R.; Boschloo, G.; Herrmann, A.; Müllen, K.; Hagfeldt, A., J. Phys. Chem. C 2007, 111, 15137.

(22) Turrisi, R.; Sanguineti, A.; Sassi, M.; Savoie, B.; Takai, A.; Patriarca, G. E.; Salamone, M. M.; Ruffo, R.; Vaccaro, G.; Meinardi, F.; Marks, T. J.; Facchetti, A.; Beverina, L., J. Mater. Chem. A 2015, 3, 8045.

(23) (a) Feiler, L.; Langhals, H.; Polborn, K., Liebigs Ann. Chem. 1995, 1995, 1229; (b) Tomizaki, K. Y.; Thamyongkit, P.; Loewe, R. S.; Lindsey, J. S., Tetrahedron 2003, 59, 1191.

(24) Chen, L. C.; Zhang, K. C.; Zhu, L. L.; Xiao, Y., Ind. Eng. Chem. Res. 2015, 54, 12699.

(25) Quante, H.; Müllen, K., Angew. Chem. Int. Ed. 1995, 34, 1323.

(26) Keerthi, A.; Liu, Y.; Wang, Q.; Valiyaveettil, S., Chem. Eur. J. 2012, $18,11669$.

(27) Sahoo, D.; Sharma, V.; Roy, R.; Varghese, N.; Mohanta, K.; Koner, A. L., Chem. Commun. 2019, 55, 103.

(28) Costabel, D.; Skabeev, A.; Nabiyan, A.; Luo, Y.; Max, J. B.; Rajagopal, A.; Kowalczyk, D.; Dietzek, B.; Wächtler, M.; Görls, H.; Ziegenbalg, D.; Zagranyarski, Y.; Streb, C.; Schacher, F. H.; Peneva, K., Chem. Eur. J. 2021, 27, 4081.

(29) Sharma, V.; Sahoo, D.; Chandra, F.; Koner, A. L., Chemistryselect 2017, 2, 11747.

(30) Weber, S.; Hofinger, J.; Rath, T.; Reinfelds, M.; Pfeifer, D.; Borisov, S. M.; Fürk, P.; Amenitsch, H.; Scharber, M. C.; Trimmel, G., Mater. Adv. 2020, 1, 2095.

(31) Pal, K.; Sharma, V.; Sahoo, D.; Kapuria, N.; Koner, A. L., Chem. Commun. 2018, 54, 523.

(32) Sharma, V.; Chandra, F.; Sahoo, D.; Koner, A. L., Eur. j. Org. Chem. 2017, 2017, 6901.

(33) Li, C.; Schöneboom, J.; Liu, Z.; Pschirer, N. G.; Erk, P.; Herrmann, A.; Müllen, K., Chem. Eur. J. 2009, 15, 878.

(34) Kazantsev, R. V.; Dannenhoffer, A. J.; Aytun, T.; Harutyunyan, B.; Fairfield, D. J.; Bedzyk, M. J.; Stupp, S. I., Chem 2018, 4, 1596.

(35) Zagranyarski, Y.; Chen, L.; Zhao, Y.; Wonneberger, H.; Li, C.; Müllen, K., Org. Lett. 2012, 14, 5444.

(36) Lin, Z.; Li, C.; Meng, D.; Li, Y.; Wang, Z. H., Chem. Asian J. 2016, 11, 2695.

(37) Feng, J.; Fu, H.; Jiang, W.; Zhang, A.; Ryu, H. S.; Woo, H. Y.; Sun, Y.; Wang, Z., ACS Appl. Mater. Interfaces 2020, 12, 29513.

(38) Zhao, X.; Chen, X.; Yuan, Z.; Zhang, H.; Luo, G.; Hu, Y.; Chen, Y., Dyes Pigm. 2020, 173, 107930.

(39) Langhals, H.; Schönmann, G.; Feiler, L., Tetrahedron Lett. 1995, 36,6423

(40) Roznyatovskiy, V. V.; Gardner, D. M.; Eaton, S. W.; Wasielewski, M. R., Org. Lett. 2014, 16, 696.

(41) Odobel, F.; Séverac, M.; Pellegrin, Y.; Blart, E.; Fosse, C.; Cannizzo, C.; Mayer, C. R.; Elliott, K. J.; Harriman, A., Chem. Eur. J. 2009, 15, 3130.

(42) Lefler, K. M.; Kim, C. H.; Wu, Y.-L.; Wasielewski, M. R., J. Phys. Chem. Lett. 2014, 5, 1608.

(43) Tomizaki, K.; Loewe, R. S.; Kirmaier, C.; Schwartz, J. K.; Retsek, J. L.; Bocian, D. F.; Holten, D.; Lindsey, J. S., J. Org. Chem. 2002, 67, 6519. 
(44) Haase, M.; Hubner, C. G.; Reuther, E.; Herrmann, A.; Mullen, K.; Basche, T., J. Phys. Chem. B 2004, 108, 10445.

(45) Hestand, N. J.; Spano, F. C., Chem. Rev. 2018, 118, 7069.

(46) Papadopoulos, I.; Gutiérrez-Moreno, D.; McCosker, P. M.; Casillas, R.; Keller, P. A.; Sastre-Santos, Á.; Clark, T.; Fernández-Lázaro, F.; Guldi, D. M., J. Phys. Chem. A 2020, 124, 5727.

(47) Czar, M. F.; Breitgoff, F. D.; Sahoo, D.; Sajid, M.; Ramezanian, N.; Polyhach, Y.; Jeschke, G.; Godt, A.; Zenobi, R., J. Phys. Chem. Lett. 2019, 10, 6942.

(48) Song, H.; Zhao, H.; Guo, Y.; Philip, A. M.; Guo, Q.; Hariharan, M.; Xia, A., J. Phys. Chem. C 2020, 124, 237.

(49) Hu, G. F.; Kang, H. S.; Mandal, A. K.; Roy, A.; Kirmaier, C.; Bocian, D. F.; Holten, D.; Lindsey, J. S., RSC Adv. 2018, 8, 23854.

(50) Alexy, E. J.; Yuen, J. M.; Chandrashaker, V.; Diers, J. R.; Kirmaier, C.; Bocian, D. F.; Holten, D.; Lindsey, J. S., Chem. Commun. 2014, $50,14512$.

(51) Zheng, D. D.; Oskouei, M. R.; Sanders, H. J.; Qian, J. H.; Williams, R. M.; Brouwer, A. M., Photochem. Photobiol. Sci. 2019, 18, 524.

(52) Jiao, C.; Huang, K.-W.; Chi, C.; Wu, J., J. Org. Chem. 2011, 76, 661.

(53) Kohl, C.; Becker, S.; Müllen, K., Chem. Commun. 2002, 23, 2778.

(54) Yarnell, J. E.; Chakraborty, A.; Myahkostupov, M.; Wright, K. M.; Castellano, F. N., Dalton Trans. 2018, 47, 15071.

(55) Zhao, Y. J.; Duan, R. M.; Zhao, J. Z.; Li, C., Chem. Commun. 2018, 54, 12329.

(56) Zhang, X.; Elmali, A.; Duan, R. M.; Liu, Q. Y.; Ji, W.; Zhao, J. Z.; Li, C.; Karatay, A., Phys. Chem. Chem. Phys. 2020, 22, 6376.

(57) Cai, Y.; Ni, D. Q.; Cheng, W. Y.; Ji, C. D.; Wang, Y. L.; Mullen, K.; Su, Z. Q.; Liu, Y.; Chen, C. Y.; Yin, M. Z., Angew. Chem. Int. Ed. 2020, 59, 14014.

(58) Ding, N.; Liu, K.; Qi, Y.; Shang, C.; Chang, X.; Fang, Y., Sens. Actuators, $B$ 2021, 340, 129964.

(59) Huang, L.; Tam-Chang, S.-W.; Seo, W.; Rove, K., Adv. Mater. 2007, 19, 4149.

(60) Roy, R.; Bhowal, R.; Sharma, V.; Chopra, D.; Koner, A. L., J. Mater. Chem. C 2021, 9, 1778.

(61) Chen, X.; Wang, Y. N.; Rong, R. X.; Zhao, C. M.; Li, X. L.; Wang, K. R., Dyes Pigm. 2019, 160, 779.

(62) Zhao, C. M.; Wang, K. R.; Wang, C.; He, X.; Li, X. L., ACS Macro Lett. 2019, 8, 381.

(63) (a) Li, C.; Yan, H.; Zhang, G. F.; Gong, W. L.; Chen, T.; Hu, R.; Aldred, M. P.; Zhu, M. Q., Chem. Asian J. 2014, 9, 104; (b) Li, C.; Yan, H.; Zhao, L. X.; Zhang, G. F.; Hu, Z.; Huang, Z. L.; Zhu, M. Q., Nat. Commun. 2014, 5,1 .

(64) Liou, J. X.; Xin, B.; Li, C.; Gong, W. L.; Huang, Z. L.; Tang, B. Z.; Zhu, M. Q., J. Mater. Chem. C 2017, 5, 9339.

(65) Gong, W. L.; Xiong, Z. J.; Xin, B.; Yin, H.; Duan, J. S.; Yan, J.; Chen, T.; Hua, Q. X.; Hu, B.; Huang, Z. L.; Zhu, M. Q., J. Mater. Chem. C 2016, 4, 2498.

(66) Ji, C.; Lai, L.; Li, P.; Wu, Z.; Cheng, W.; Yin, M., Aggregate 2021, 1.

(67) Lefler, K. M.; Co, D. T.; Wasielewski, M. R., J. Phys. Chem. Lett. 2012, 3, 3798.

(68) Dannenhoffer, A.; Sai, H.; Huang, D. X.; Nagasing, B.; Harutyunyan, B.; Fairfield, D. J.; Aytun, T.; Chin, S. M.; Bedzyk, M. J.; de la Cruz, M. O.; Stupp, S. I., Chem. Sci. 2019, 10, 5779.

(69) Pal, K.; Sharma, V.; Koner, A. L., Chem. Commun. 2017, 53, 7909.

(70) Kapuria, N.; Sharma, V.; Kumar, P.; Koner, A. L., J. Mater. Chem. C 2018, 6, 11328.

(71) Lewandowska, U.; Zajaczkowski, W.; Corra, S.; Tanabe, J.; Borrmann, R.; Benetti, E. M.; Stappert, S.; Watanabe, K.; Ochs, N. A. K.; Schaeublin, R.; Li, C.; Yashima, E.; Pisula, W.; Müllen, K.; Wennemers, H., Nat. Chem. 2017, 9, 1068.
(72) Zafer, C.; Kus, M.; Turkmen, G.; Dincalp, H.; Demic, S.; Kuban, B.; Teoman, Y.; Icli, S., Sol. Energy Mater. Sol. Cells 2007, 91, 427.

(73) O’Regan, B.; Grätzel, M., Nature 1991, 353, 737.

(74) Menekse, K.; Renner, R.; Mahlmeister, B.; Stolte, M.; Würthner, F., Org. Mater. 2020, 02, 229.

(75) Tong, Y.; Xiao, Z.; Du, X.; Zuo, C.; Li, Y.; Lv, M.; Yuan, Y.; Yi, C.; Hao, F.; Hua, Y.; Lei, T.; Lin, Q.; Sun, K.; Zhao, D.; Duan, C.; Shao, X.; Li, W.; Yip, H.-L.; Xiao, Z.; Zhang, B.; Bian, Q.; Cheng, Y.; Liu, S.; Cheng, M.; Jin, Z.; Yang, S.; Ding, L., Sci. China Chem. 2020, 63, 758.

(76) (a) Cremer, J.; Mena-Osteritz, E. M.; Pschierer, N. G.; Mullen, K.; Bauerle, P., Org. Biomol. Chem. 2005, 3, 985; (b) Cremer, J.; Bäuerle, P., Eur. J. Org. Chem. 2005, 2005, 3715; (c) Cremer, J.; Bäuerle, P., J. Mater. Chem. C 2006, 16, 874.

(77) Grätzel, M., J. Photochem. Photobiol., C 2003, 4, 145.

(78) https://www.gamry.com/application-notes/physechem/dsscdye-sensitized-solar-cells/ (accessed July 19, 2021).

(79) Li, C.; Wonneberger, H., Adv. Mater 2012, 24, 613.

(80) (a) Ferrere, S.; Gregg, B. A., New J. Chem. 2002, 26, 1155; (b) Ferrere, S.; Gregg, B. A., J. Phys. Chem. B 2001, 105, 7602.

(81) (a) Le Pleux, L.; Smeigh, A. L.; Gibson, E.; Pellegrin, Y.; Blart, E.; Boschloo, G.; Hagfeldt, A.; Hammarstrom, L.; Odobel, F., Energy Environ. Sci. 2011, 4, 2075; (b) Mikroyannidis, J. A.; Stylianakis, M. M.; Suresh, P.; Roy, M. S.; Sharma, G. D., Energy Environ. Sci. 2009, 2, 1293.

(82) (a) Nattestad, A.; Mozer, A. J.; Fischer, M. K. R.; Cheng, Y. B.; Mishra, A.; Bäuerle, P.; Bach, U., Nat. Mater. 2010, 9, 31; (b) Weidelener, M.; Powar, S.; Kast, H.; Yu, Z.; Boix, P. P.; Li, C.; Mullen, K.; Geiger, T.; Kuster, S.; Nuesch, F.; Bach, U.; Mishra, A.; Bauerle, P., Chem. Asian J. 2014, 9, 3251; (c) Qin, R.; Guo, D.; Li, M.; Li, G.; Bo, Z.; Wu, J., ACS Appl. Energy Mater. 2019, 2, 305.

(83) Li, W. Q.; Zhou, H. P.; Nawaz, M. A. H.; Niu, N.; Yang, N.; Ren, J.; Yu, C., Anal. Methods 2020, 12, 5353.

(84) Garcia-Calvo, J.; Robson, J. A.; Torroba, T.; Wilton-Ely, J. D. E. T., Chem. Eur. J. 2019, 25, 14214.

(85) Wang, J. F.; Zhao, C. M.; Yang, J. X.; He, X.; Li, X. L.; Li, J. M.; Wang, K. R., Chem. Commun. 2021, 57, 2776.

(86) Liu, J. X.; Xin, B.; Li, C.; Xie, N. H.; Gong, W. L.; Huang, Z. L.; Zhu, M. Q., ACS Appl. Mater. Interfaces 2017, 9, 10338.

(87) Liu, J.-X.; Xin, B.; Li, C.; Gong, W.-L.; Huang, Z.-L.; Tang, B.-Z.; Zhu, M.-Q., J. Mater. Chem. C 2017, 5, 9339.

(88) Busto, N.; Garcia-Calvo, J.; Cuevas, J. V.; Herrera, A.; Mergny, J. L.; Pons, S.; Torroba, T.; Garcia, B., Bioorg. Chem. 2021, 108, 104660.

(89) Cai, Y.; Wei, Z.; Song, C.; Tang, C.; Han, W.; Dong, X., Chem. Soc. Rev. 2019, 48, 22.

(90) Mengji, R.; Acharya, C.; Vangala, V.; Jana, A., Chem. Commun. 2019, 55, 14182.

(91) Jana, A.; Bai, L. Y.; Li, X.; Agren, H.; Zhao, Y. L., ACS Appl. Mater. Interfaces 2016, 8, 2336.

(92) Weingarten, A. S.; Kazantsev, R. V.; Palmer, L. C.; McClendon, M.; Koltonow, A. R.; Samuel, A. P. S.; Kiebala, D. J.; Wasielewski, M. R.; Stupp, S. I., Nat. Chem. 2014, 6, 964.

(93) Weigarten, A. S.; Dannenhoffer, A. J.; Kazantsev, R. V.; Sai, H.; Huang, D. X.; Stupp, S. I., J. Am. Chem. Soc. 2018, 140, 4965.

(94) Christ, T.; Petzke, F.; Bordat, P.; Herrmann, A.; Reuther, E.; Müllen, K.; Basché, T., J. Lumin. 2002, 98, 23.

(95) Aryal, G. H.; Huang, L. M.; Hunter, K. W., RSC Adv. 2016, 6, 82566.

(96) Aryal, G. H.; ViK, R.; Assaf, K. I.; Hunter, K. W.; Huang, L. M.; Jayawickramarajah, J.; Nau, W. M., Chemistryselect 2018, 3, 4699. 